\title{
Self-management in Epilepsy
}

Citation for published version (APA):

Leenen, L. A. M. (2018). Self-management in Epilepsy: The Goal is: "Live with a Z(s)mile". [Doctoral Thesis, Maastricht University]. Datawyse / Universitaire Pers Maastricht.

https://doi.org/10.26481/dis.20180907II

Document status and date:

Published: 01/01/2018

DOI:

10.26481/dis.20180907II

Document Version:

Publisher's PDF, also known as Version of record

\section{Please check the document version of this publication:}

- A submitted manuscript is the version of the article upon submission and before peer-review. There can be important differences between the submitted version and the official published version of record.

People interested in the research are advised to contact the author for the final version of the publication, or visit the DOI to the publisher's website.

- The final author version and the galley proof are versions of the publication after peer review.

- The final published version features the final layout of the paper including the volume, issue and page numbers.

Link to publication

\footnotetext{
General rights rights.

- You may freely distribute the URL identifying the publication in the public portal. please follow below link for the End User Agreement:

www.umlib.nl/taverne-license

Take down policy

If you believe that this document breaches copyright please contact us at:

repository@maastrichtuniversity.nl

providing details and we will investigate your claim.
}

Copyright and moral rights for the publications made accessible in the public portal are retained by the authors and/or other copyright owners and it is a condition of accessing publications that users recognise and abide by the legal requirements associated with these

- Users may download and print one copy of any publication from the public portal for the purpose of private study or research.

- You may not further distribute the material or use it for any profit-making activity or commercial gain

If the publication is distributed under the terms of Article $25 \mathrm{fa}$ of the Dutch Copyright Act, indicated by the "Taverne" license above, 


\section{SELF-MANAGEMENT IN EPILEPSY \\ THE GOAL IS \\ 'LIVE WITH A Z(S)MILE'}

Loes AM Leenen 
The studies presented in this thesis were conducted at the Academic Centre Kempenhaeghe in collaboration with the School of Health Profession Education (SHE); the School for Mental Health and Neuroscience (MEHNS) and School CAPHRI: Care and Public Health Research Institute

\section{Academisch Centrum voor Epileptologie}

Kempenhaeghe \& Maastricht UMC.

\section{Maastricht University}

The research presented in this thesis was funded by the Netherlands Organisation for Health Research and Development (ZonMW) project 101883601

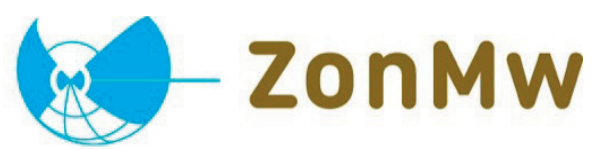

Printing and dissemination of this thesis was financially supported by CAPHRI, Kempenhaeghe, Eisai and UCB Pharma B.V.

\section{Kempenhaeghe}

\section{Eisai}

(C) copyright Loes Leenen, Maastricht 2018

Printing: Datawyse | Universitaire Pers Maastricht 


\title{
Self-management in Epilepsy
}

\section{The Goal is: "Live with a Z(s)mile"}

\author{
PROEFSCHRIFT \\ Ter verkrijging van de graad van doctor aan de Universiteit Maastricht, \\ op gezag van de Rector Magnificus, Prof. dr. Rianne M. Letschert \\ volgens het besluit van de het College van Decanen, \\ het openbaar te verdedigen in \\ op 7 September 2018, om 10.00u \\ door \\ Louisa (Loes) Anna Maria Leenen
}




\section{Promotores}

Prof. dr. H.J.M. Majoie

Prof. dr. mr. S.M.A.A. Evers

Prof. dr. C.M. van Heugten

\section{Beoordelingscommissie}

1. Prof. dr. M.E. de Vugt, (voorzitter)

2. Prof. dr. P.A.J.M Boon Academisch Centrum Kempenhaeghe Heeze \& Universitair Ziekenhuis Gent

3. Prof. dr. C.G. Faber

4. Dr. A.T.G. Paulus

5. Prof. dr. W.J.M. Scholte op Reimer Academisch Medisch Centrum Amsterdam \& Universiteit van Amsterdam. 


\section{Contents}

CHAPTER 1 General Introduction \& Outline of this thesis

CHAPTER 2 (Cost)-effectiveness of a multi-component intervention for adults with epilepsy: Study protocol of a Dutch randomized controlled trial (ZMILE study).

BMC Neurology 2014; 14:255

CHAPTER 3 Are People with Epilepsy using eHealth-tools?

Epilepsy \& Behavior 2016; 64: 268-272

CHAPTER 4 Process Evaluation of a Multi-Component Self-Management

Intervention for Adults with Epilepsy (ZMILE study).

Epilepsy \& Behavior 2017; 73: 64-70

CHAPTER 5 An Economic Evaluation of a Multi-component Self-Management Intervention for Adults with Epilepsy (ZMILE study)

Epilepsia 2017; 58: 1398-1408

CHAPTER 6 Effectiveness of a Multi-component Self-Management Intervention for Adults with Epilepsy (ZMILE study): A Randomized Controlled Trial

Epilepsy \& Behavior 2018; 80: 259-265

CHAPTER 7 General Discussion

Summary

Samenvatting

Valorisation

Addendum

List of publications

Dankwoord

About the author 



\section{1}

General Introduction \& Outline of this thesis

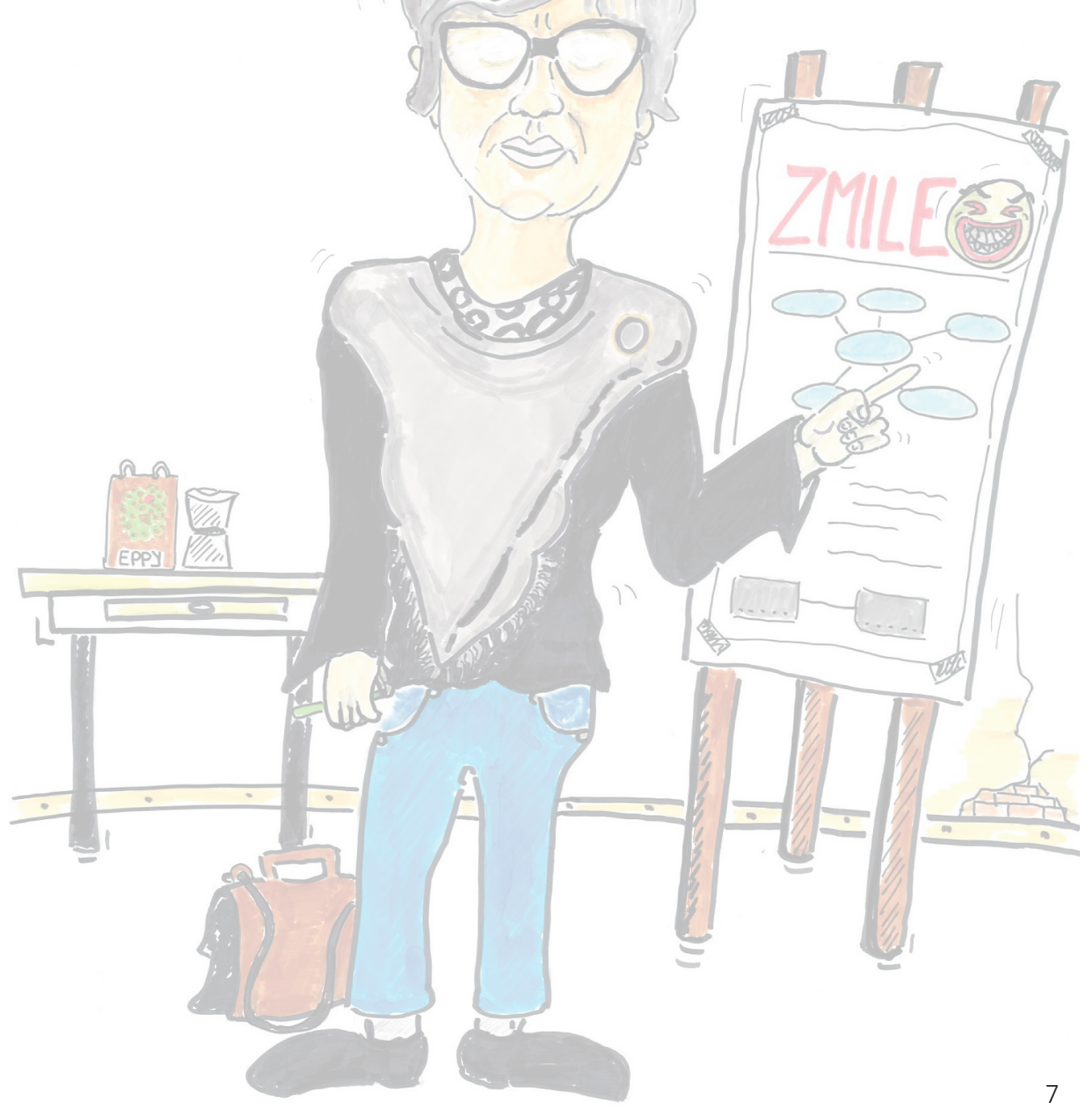





\section{INTRODUCTION}

\section{Epilepsy}

Epilepsy is a neurological disease characterized by recurrent unprovoked seizures. Since seizures are the result of sudden excessive electrical discharges in a group of brain cells, they vary in their manifestations. This variation depends on where in the brain the disturbance starts and how it spreads. ${ }^{1}$ In epileptic seizures, transient symptoms can occur, such as loss of awareness or consciousness and disturbances of movement, sensation, mood or mental function. ${ }^{2}$

Worldwide, between $50^{1}$ and 70 million people ${ }^{3}$ are estimated to have epilepsy. Incidence of epilepsy varies with a high rate in early childhood, a low rate in early adulthood and again a high rate in people over 65 years. ${ }^{2}$ In Europe, there are 400,000 new cases every year. ${ }^{4}$ In Dutch general practice, the incidence rate of epilepsy in 2016 was 0.8 and the prevalence rate 10.7 per 1000 patient years. ${ }^{5}$

\section{Treatment of Epilepsy}

In cases of epilepsy, anti-epileptic drugs (AED) are the treatment of first choice. The primary goal of AED treatment is to achieve a balance between seizure control and tolerability (i.e. avoiding side-effect); this is important for optimal adherence. ${ }^{6}$ About $70 \%$ of newly diagnosed children and adults with epilepsy can be treated successfully by one or more AED. ${ }^{1}$ For the $30 \%$ of persons with epilepsy (PWE) who are not seizure-free, there are some alternative non-pharmacological treatment options, such as epilepsy surgery, vagus nerve stimulation, deep brain stimulation or the ketogenic diet. ${ }^{2,7}$

\section{Burden of Epilepsy}

\section{Psychosocial consequences}

Living with epilepsy affects a person's personality and interferes with many aspects of everyday life including education, leisure and occupational activities. ${ }^{8}$ Compared to the general population, people with epilepsy suffer more from depression and anxiety, feel more stigmatized $^{9,10}$, have a higher mortality rate ${ }^{11}$ and are more prone to iatrogenic consequences of a lifetime of AED usage, such as osteoporosis. ${ }^{12}$ Thus, these psychological and emotional consequences are reflected in a reduced quality of life (QOL). ${ }^{13,14}$

\section{Economic consequences}

People with epilepsy are often confronted by limitations, including reduced access to health and life insurance, being denied the opportunity to obtain a driving license, and barriers to entering particular occupations. ${ }^{8}$ The unemployment rate among people with epilepsy is twice as high as in the general population. ${ }^{15,16}$ This is not only a personal economic consequence, it effects the society as a whole. Persons with (uncontrolled) epilepsy 
heavily depend on informal care (family and friends) and health care professionals (neurologists, nurse practitioners, nurses, social workers, psychologists etc.). Epilepsy is, therefore, a costly disease, both in terms of health care costs and productivity losses. The healthcare costs for PWE were about 249 million euros in 2011, representing $4.8 \%$ of the healthcare costs spent on neurological conditions or $0.3 \%$ of the total healthcare cost. ${ }^{17}$

In summary: people with epilepsy do not only have to manage the medical aspects of their disease; they also have to deal with life style changes and the psychosocial burden with a high economic impact.

\section{Self-management}

Since most PWE live at home with their family, it is important for them to be able to manage their own lives. Dealing with the medical and psychosocial consequences of having a disease such as epilepsy, is a major task which requires specific skills. Self-management refers to the ability of persons to deal with medical, physical and psychosocial consequences of a chronic condition and their impact on daily life. ${ }^{18}$

The consequences of poor self-management, e.g. not taking AED or irregular sleeping patterns, are not always directly observable and, therefore, not transparent to patients. Additionally, PWE are not always aware that they missed a dose of their AED. ${ }^{19}$ There is thus a need for an intervention aimed at supporting PWE and their relatives, in enhancing self-management skills.

According to Faber (2013), self-management education alone is not enough to improve self-efficacy, which is at least one of the mechanisms responsible for improvements in health outcomes, as demonstrated in self-management interventions developed for other chronic conditions. ${ }^{20,21}$ Self-efficacy is defined as the confidence one has to carry out behaviours necessary to reach a desired goal. ${ }^{22}$

Self-management education should be accompanied by peer-support, goal-setting skills, involvement in one's treatment plan and self-monitoring. ${ }^{23}$ The existing self-management interventions, however, focus mainly on just one component of self-management, e.g. self-management education ${ }^{24}$ or compliance. ${ }^{25}$

Self-management in epilepsy is still in its infancy. A systematic review on care delivery and self-management strategies concluded that further quality research was needed, especially on the subject of self-management. ${ }^{26}$

\section{The Multi-Component Self-Management Intervention for adults with epilepsy}

Following the suggestion by Faber, we developed a group-based, multi-component selfmanagement intervention ( $\mathrm{MCl}$ ) for PWE and their relatives. The $\mathrm{MCl}$ is based on the idea that in order to manage the physical, medical and psychosocial consequences of their disease, PWE must not only become familiar with risk-management and self-monitoring (by means of eHealth-tools). Emphasis should also be placed on changing behaviour by improving pro-active coping and stimulating shared decision-making, resulting in 
concordance. Concordance is a broader concept than adherence, since it refers to a consensual agreement about one's treatment plan, and not to an informed consent about treatment. ${ }^{19}$ One of the main objectives of the Dutch government is to establish the quality of healthcare by means of developing and applying eHealth. In the $\mathrm{MCl}$, therefore, self-monitoring using eHealth-tools was discussed. ${ }^{27}$

\section{AIM OF THE THESIS}

The aim of the ZMILE study was to develop a multi-component, self-management intervention and to establish the feasibility and (cost)-effectiveness of this intervention.

As a result, this thesis addresses the following research questions:

1. Are eHealth-tools usable tools to address self-management in persons with epilepsy?

2. Is a group-based, epilepsy-specific self-management intervention feasible?

3. Is a group-based, epilepsy-specific self-management intervention (cost)-effective?

- Is a group-based, epilepsy-specific self-management intervention compared to care as usual more effective in increasing self-efficacy and other patient reported outcomes?

- Is a group-based, epilepsy-specific self-management intervention preferable to care as usual from a societal perspective in terms of costs and effects?

\section{OUTLINE OF THE THESIS}

This thesis presents the results of the ZMILE-study (Zelf-Management Interventie Leven met Epilepsie) and a study towards the use of eHealth(-tools) in persons with epilepsy.

Chapter two describes the study protocol of the multi-component intervention for adults with epilepsy (ZMILE-study).

Chapter three presents the results of a survey among people with epilepsy and their carers about whether they have access to hardware and if they perceive eHealth-tools as useful for self-management purposes.

Chapter four describes the outcomes of the process evaluation of the self-management intervention for adults with epilepsy and if appropriate their relatives.

Chapter five presents the outcomes of the randomized controlled trial evaluating the cost-effectiveness of the multi-component intervention.

Chapter six presents the outcomes of the randomized controlled trial evaluating the clinical effectiveness of the multi-component intervention.

Chapter seven presents a general discussion on the main findings of the studies and elaborates on clinical implications and further research. 


\section{REFERENCES}

1. Epilepsy Fact sheet N*999. In: World Health Organisation; 2017; http://www.who.int/mediacentre/factsheets/fs999/en/

2. Kwan P, Brodie MJ. Early identification of refractory epilepsy. N Engl J Med 2000;342: 314-9.

3. Ngugi AK, Kariuki SM, Bottomley C, Kleinschmidt I, Sander JW, Newton CR. Incidence of epilepsy: a systematic review and meta-analysis. Neurology 2011;77: 1005-12.

4. Baulac M, de Boer H, Elger C, Glynn M, Kälviäinen R, Little A, et al. Epilepsy priorities in Europe: A report of the ILAE-IBE Epilepsy Advocacy Europe Task Force. Epilepsia 2015;56: 1687-1695.

5. Incidence and prevalence numbers. In: Netherlands Institute for health services research; https://www.nivel.nl/nl/nzr/incidenties-en-prevalenties

6. Perucca P, Carter J, Vahle V, Gilliam FG. Adverse antiepileptic drug effects: toward a clinically and neurobiologically relevant taxonomy. Neurology 2009; 72: 1223-9..

7. Strzelczyk A, Reese JP, Dodel R, Hamer HM. Cost of epilepsy: a systematic review. Pharmacoeconomics 2008;26: 463-76.

8. Baker GA. The psychosocial burden of epilepsy. Epilepsia 2002;43 Suppl 6: 26-30.

9. de Boer HM, Mula M, Sander JW. The global burden and stigma of epilepsy. Epilepsy Behav. 2008;12: 5406.

10. Dilorio C, Yeager K. The Epilepsy Self-Efficacy Scale. In: Strickland O, Dilorio C, editors. Measurement of nursing outcomes: Self Care and Coping. 2nd ed. New York: Springer Publishing Company; 2003, p. 40-51.

11. Tomson T, Beghi E, Sundqvist A, Johannessen SI. Medical risks in epilepsy: a review with focus on physical injuries, mortality, traffic accidents and their prevention. Epilepsy Res 2004;60: 1-16.

12. Beerhorst K, Schouwenaars FM, Tan IY, Aldenkamp AP. Epilepsy: fractures and the role of cumulative antiepileptic drug load. Acta Neurol Scand 2012;125: 54-9.

13. Jacoby A, Baker GA. Quality-of-life trajectories in epilepsy: a review of the literature. Epilepsy Behav 2008;12: 557-71.

14. Taylor RS, Sander JW, Taylor RJ, Baker GA. Predictors of health-related quality of life and costs in adults with epilepsy: a systematic review. Epilepsia 2011;52: 2168-80.

15. Pato Pato A, Cebrián Pérez E, Cimas Hernando I, Lorenzo González JR, Rodríguez Constenla I, Gude Sampedro F. Analysis of direct, indirect, and intangible costs of epilepsy. Neurología (English Edition) 2011;26: 32-38.

16. Smeets VM, van Lierop BA, Vanhoutvin JP, Aldenkamp AP, Nijhuis FJ. Epilepsy and employment: literature review. Epilepsy Behav 2007;10: 354-62.

17. In: Volksgezondheidenzorg.info; https://www.volksgezondheidenzorg.info/onderwerp /epilepsie/cijferscontext/huidige-situatie

18. Barlow J, Wright C, Sheasby J, Turner A, Hainsworth J. Self-management approaches for people with chronic conditions: a review. Patient Education and Counseling 2002;48: 177-187.

19. Horne R. Compliance, adherence, and concordance: implications for asthma treatment. Chest 2006;130: 65S-72S.

20. Lorig KR, Holman H. Self-management education: history, definition, outcomes, and mechanisms. Ann Behav Med 2003;26: 1-7.

21. Marks R, Allegrante JP, Lorig K. A review and synthesis of research evidence for self-efficacy-enhancing interventions for reducing chronic disability: implications for health education practice (part II). Health Promot Pract 2005;6: 148-56.

22. Benight CC, Bandura A. Social cognitive theory of posttraumatic recovery: the role of perceived self-efficacy. Behav Res Ther 2004;42: 1129-48.

23. Faber M, Harmsen M, van der Burg S, van der Weijden T. Gezamenlijke besluitvorming \& Zelfmanagement: Een literatuuronderzoek naar de effectiviteit en naar voorwaarden voor succes. In: Scientific Institute for Quality of Healthcare (IQ healthcare); 2013; https://www.raadrvs.nl/uploads/docs/Achtergrondstudie_Gezamenlijkebesluitvorming_en_Zelfmanagement.pdf 
24. May TW, Pfafflin M. The efficacy of an educational treatment program for patients with epilepsy (MOSES): results of a controlled, randomized study. Modular Service Package Epilepsy. Epilepsia 2002;43: 539-49.

25. Eatock J, Baker GA. Managing patient adherence and quality of life in epilepsy. Neuropsychiatr Dis Treat 2007;3: 117-31.

26. Bradley PM, Lindsay B. Care delivery and self-management strategies for adults with epilepsy. Cochrane Database Syst Rev 2008: CD006244.

27. Raad voor de Volksgezondheid (RVZ). De participerende patiënt In: Raad voor de Volgsgezondheid en Zorg; 2013. 

(Cost)-effectiveness of a multi-component intervention for adults with epilepsy: Study protocol of a Dutch randomized controlled trial

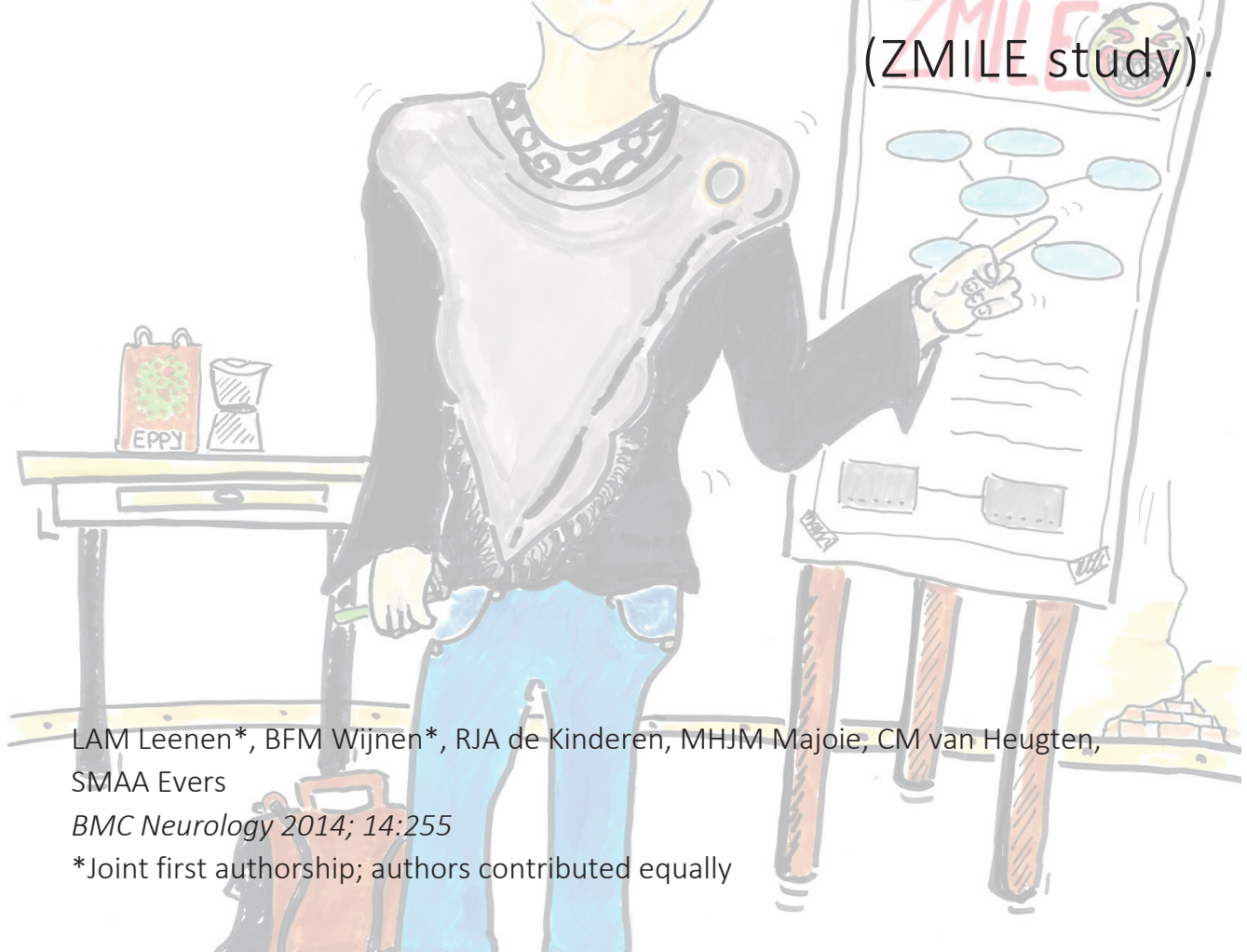




\section{ABSTRACT}

\section{Background}

In patients with epilepsy, poor adherence to anti-epileptic drugs has been shown to be the most important cause of poorly controlled epilepsy. Furthermore, it has been noted that the quality of life among patients with epilepsy can be improved by counselling and treatments aimed at increasing their self-efficacy and concordance, thus stimulating selfmanagement skills. However, there is a need for evidence on the effectiveness of such programs, especially within epilepsy care. Therefore, we have developed a multi-component intervention ( $\mathrm{MCl}$ ) which combines a self-management/education program with eHealth interventions. Accordingly, the overall objective of this study is to assess the (cost)effectiveness and feasibility of the $\mathrm{MCl}$, aiming to improve self-efficacy and concordance in patients with epilepsy.

\section{Methods}

A randomized controlled trial in two parallel groups will be conducted to compare the $\mathrm{MCl}$ with a control (care as usual) condition in epilepsy patients. One hundred eligible epilepsy patients will be recruited and allocated to either the intervention or control group. The intervention group will receive the $\mathrm{MCl}$ consisting of a self-management/education program of six meetings, including e-Health interventions, and will be followed for 12 months. The control group will receive care as usual and will be followed for 6 months, after which patients will be offered the possibility of participating in the $\mathrm{MCl}$. The study will consist of three parts: 1) a clinical effectiveness study, 2) a cost-effectiveness study, and 3) process evaluation. The primary outcome will be self-efficacy. Secondary outcomes include adherence, side effects, change in seizure severity \& frequency, improved quality of life, proactive coping, and societal costs. Outcome assessments will be done using questionnaires at baseline and after 3, 6, 9, and 12 months (last two applicable only for the intervention group).

\section{Discussion}

In times of budget constraints, $\mathrm{MCl}$ could be a valuable addition to the current healthcare provision for epilepsy, as it is expected that higher concordance and self-efficacy will result in reduced use of healthcare resources and an increased QOL. Accordingly, this study is aimed helping patients to be their own provider of health care, shifting epilepsy management from professionals to self-care by patients equipped with appropriate skills and tools. 


\section{BACKGROUND}

Epilepsy is a chronic disorder of the brain, characterized by recurrent seizures. Seizures are the result of sudden, excessive electrical discharges in a group of brain cells. Different parts of the brain can be the site of such discharges, resulting in a variety of clinical manifestations. $^{1}$

Epilepsy has a considerable psychological and emotional impact, which is strongly reflected in a reduced quality of life ( $\mathrm{QOL}$ ) for patients suffering from epilepsy. ${ }^{2,3}$ Living with seizures is likely to affect patients' daily activities, as it interferes with many aspects of everyday life. Furthermore, epilepsy has been shown to have a large economic impact on society as a whole. ${ }^{4,5}$ For example, the unemployment rate among epileptic patients is at least twice as high as in the general population. ${ }^{6-8}$

Recent studies have shown that up to $70-80 \%$ of newly diagnosed epilepsy patients can be treated successfully (i.e. seizures completely controlled) with anti-epileptic drugs $(A E D)^{9-11}$ and it is estimated that currently more than 80,000 patients in the Netherlands are treated with AED. ${ }^{12}$ However, to achieve and maintain successful seizure control, adherence to treatment is of major importance. A systematic review argued that effective ways of helping people follow medical treatments could have far larger effects on health than any treatment itself. ${ }^{13}$ In addition, it has been recommended that the cost-effectiveness of adherence interventions should be a research priority in the field of chronic diseases. ${ }^{14}$ In epilepsy, poor adherence has been shown to be the most important cause of poorly controlled epilepsy. ${ }^{15}$ However, this study is striving to improve 'concordance' and not only 'adherence'. The crucial difference is that 'adherence' describes only the extent to which a patient takes antiepileptic drugs as prescribed with respect to dosage and dosing intervals ${ }^{16}$, while 'concordance' includes a consensual agreement about taking AEDs that has been established between patient and practitioner. ${ }^{13}$

Concordance with medical treatment is closely linked with the patients' ability to selfmanage their disease, and the latter is shown to be an important factor in determining quality of life. ${ }^{17}$ Self-management programs focus on supporting patients in coping with their chronic condition, eventually to maximize quality of life ${ }^{18}$, and have been identified as useful for individuals with chronic conditions such as asthma, heart disease, diabetes. ${ }^{18,19}$ However, due to several reasons, results of studies relating to chronic patient groups cannot be generalized to patients with epilepsy. For example, the consequences of poor self-management, i.e. not taking AED or irregular sleeping patterns, are not always directly observable; seizure deregulation can appear the same day or a couple of days later. Hence, the direct link between poor disease management and the frequency of seizures is not transparent for epilepsy patients. A recent study showed that many patients with epilepsy seem to be unaware of missed doses, indicating the need for pill dispensers and reminding/educational interventions. ${ }^{20}$

Self-efficacy, defined as the confidence to carry out behaviour necessary to reach a desired goal ${ }^{18}$ an important concept in self-management. Self-efficacy and changes in 
self-efficacy are associated with future health status and it appears that enhanced selfefficacy is at least one of the mechanisms responsible for the improvements in health status demonstrated by those attending self-management programs. ${ }^{21-23}$ Working within the field of epilepsy, Pramuka et al. ${ }^{19}$ piloted a psychosocial self-management program for epilepsy and observed a positive correlation between self-efficacy and quality of life. In addition, Amir et al. ${ }^{17}$ emphasized the possibility of increasing quality of life among patients with epilepsy by counselling and treatment aimed at increasing their self-efficacy. However, the Managing Epilepsy Well network recently concluded that too few selfmanagement programs exist in general and that there are too few evidence-based programs available. ${ }^{24}$

This study will therefore evaluate the feasibility and (cost-)effectiveness of a multicomponent intervention $(\mathrm{MCl})$, which combines a self-management/education program with e-Health interventions, aiming to improve self-efficacy and concordance in people with epilepsy, in comparison with care as usual (CAU). Hence the $\mathrm{MCl}$ focuses on increasing patients' understanding of their medical regimens, and on providing skills and tools to strengthen self-management and communication between patient and healthcare professional and increase adherence (as a proxy for concordance).

\section{METHODS}

This study will consist of three parts, each with its own research questions:

\section{Clinical effectiveness}

Is $\mathrm{MCl}$, in comparison with CAU, more effective in terms of self-efficacy and other patientreported outcomes (self-efficacy, adherence, decrease in seizure frequency \& severity, side effects of AED, controlling depression/anxiety, proactive coping, improved quality of life, and societal costs)?

Does the $\mathrm{MCl}$ have a clinical superiority over CAU in terms of a better adherence to AED?

\section{Economic evaluation}

What are the cost-effectiveness and the cost-utility of the $\mathrm{MCl}$ in comparison with $\mathrm{CAU}$ from a societal perspective?

\section{Process evaluation}

Has the $\mathrm{MCl}$ been delivered according to protocol? And if not, what are the reasons for protocol deviation? 
What are the experiences and opinions of patients, caregivers and professionals regarding the $\mathrm{MCl}$ ?

To what extent has the $\mathrm{MCl}$ impacted concordance among patients (i.e. do patients understand why it is relevant to take the $\mathrm{AED}$, and has the $\mathrm{MCl}$ impacted shared decision making by means of consensual agreement between patient and doctor regarding the medical regimen?) The control group will receive CAU as naturally as possible. The followup of patients in the intervention group will be 12 months and the follow-up of patients assigned to the control group will be 6 months, after which patients in the control group have the opportunity to receive the $\mathrm{MCl}$ outside the study (Fig. 2.1). The study has been approved by the Ethics Committee of University Hospital Maastricht, the Netherlands.

\section{Study population}

One hundred eligible epilepsy patients will be included in the study. Eligible patients are adults aged 18 or over, living at home, diagnosed with epilepsy and using AED, who understand the Dutch language, and are willing and able to use e-Health devices belonging to the $\mathrm{MCl}$. Patients will be excluded if they are not able or willing to function in group activities or when it is expected, on the basis of clinical judgment, that patients are not able to comprehend topics discussed within the $\mathrm{MCl}$ (i.e. patients with cognitive deficits).

\section{Setting \& recruitment}

This study is a collaboration between Maastricht University and the epilepsy centre Kempenhaeghe $(\mathrm{KH})$. The study will be conducted at the outpatient clinics of $\mathrm{KH}$. The first $\mathrm{MCl}$ will be offered at the outpatient clinic at the main location of $\mathrm{KH}$ (Heeze), after which the $\mathrm{MCl}$ will be offered at other outpatient clinics (i.e. Nijmegen and Maastricht). From April 2014 onwards, neurologists and nurse practitioners (NP) will recruit possible candidates for participation during consultations at the outpatient clinics of $\mathrm{KH}$. Furthermore, a press release will be published in national epilepsy magazines and on social media, and patient information leaflets will be distributed to patients in the outpatient clinics of $\mathrm{KH}$. In the press release patients are invited to send their contact information to the researchers.

When patients show interest in participating, an information meeting will be scheduled with one of the researchers (LAML or BFMW), either by phone or face-to-face, in which patients will have the opportunity to ask questions and in which inclusion and exclusion criteria will be checked either by the researchers during the meeting or afterwards in consultation with clinicians. During the meeting, patients are informed about the study and that they will be randomly assigned either to the intervention or control group. After one week researchers will contact patients who have received information and want to take part in the study to plan a visit. During this visit an informed consent form will be signed by the patient and the researcher, and patients will be allocated randomly to either the intervention or control group. 


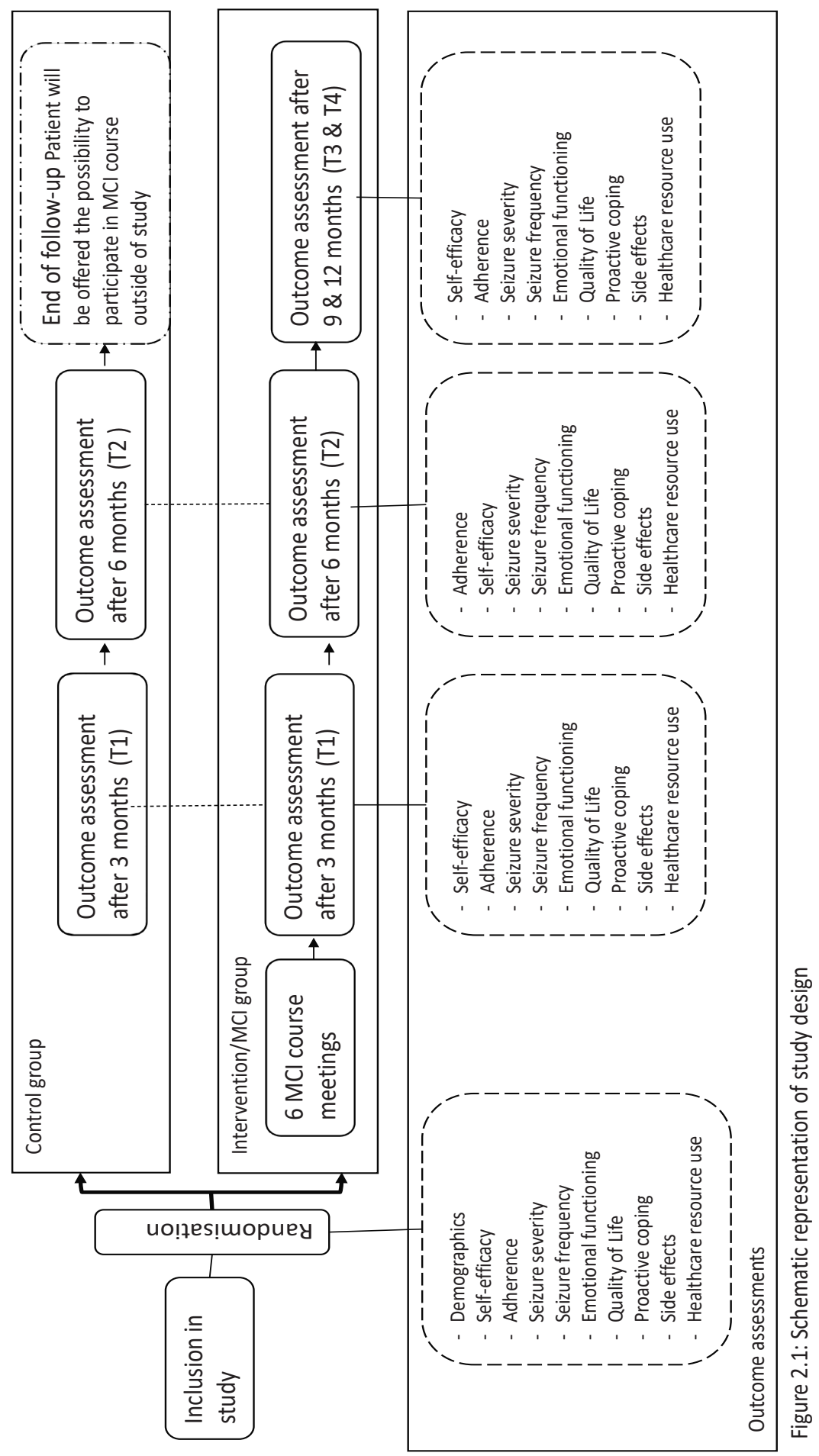




\section{Sample size}

The primary outcome variable of this study will be self-efficacy as measured on the Epilepsy Self-Efficacy Scale 33 (ESES). ${ }^{25}$ In a previous pilot study exploring the effects of a psychosocial self-management program for epilepsy patients, with self-efficacy as the primary outcome, the difference between groups was approximately 10 points, with a standard deviation of 7 points on the ESES ${ }^{19}$. Assuming that alpha $=0.05$ and power $p=$ 0.90, a minimally detectable difference of 5 points between the intervention and control groups, we will need to include 42 patients per group. Based on a dropout rate of $20 \%$ we intend to include 50 patients in each group.

\section{Randomization}

Patients will be randomized to the $\mathrm{MCl}$ (intervention group) or to the control group, in which patients will be given the opportunity to attend the $\mathrm{MCl}$ outside of the study after six months of follow-up. For parallel provision of both groups (intervention \& control), two blocks of five patients are needed at the moment of randomization. Patients will be assigned to the intervention group or the control group by means of block randomization, using blocks of 10 patients. An assistant who is not involved in the treatment nor in the trial will execute the procedure with a randomization program (www.randomization.com).

\section{Multi-component intervention (MCI)}

Self-management is a process in which patients take responsibility for changing their health behaviour by acquiring knowledge about their disease and treatment, and by managing symptoms as well as the physical and psychosocial consequences of the disease. ${ }^{26}$

This definition is a compilation of a broad range of definitions about self-management, combining physical functioning and outcomes with knowledge and the psychosocial consequences of disease. Our $\mathrm{MCl}$ is based on this idea. In order to manage symptoms or the physical and psychosocial consequences, we will try to provide patients with knowledge about self-monitoring (by use of e-health tools) and risk management. In addition, in order to change behaviour, emphasis will be put on proactive coping, concordance and goal-setting.

Patients will have the opportunity to bring one of their relatives/friends for social support, based on the fact that self-management is not only supported by healthcare providers, but also by the people surrounding persons with chronic diseases. ${ }^{26}$ The intervention is based on the self-management intervention offered in the Restore4Stroke study ${ }^{27}$ but was adjusted to make it suitable for epilepsy patients. The final version of the intervention was developed in agreement with experts in the field of self-management, coordinators of the Restore4Stroke study, clinicians, and representatives of the Epilepsy Association of the Netherlands (EVN, Epilepsie Vereniging Nederland). 
The intervention will be explained in a detailed protocol for the NP offering the intervention in the outpatient clinic and in a workbook for patients and one of their relatives/friends. In addition, NPs will receive training beforehand on motivational interviewing (MI), as a technique to empower patients to set their own sustainable goals and look into conflicting beliefs. $\mathrm{Ml}$ is defined as "a collaborative, person-centred form of guiding to elicit and strengthen motivation for change". ${ }^{28} \mathrm{Ml}$ focuses on exploring and resolving ambivalence and centres on motivational processes within the individual that facilitate change. ${ }^{29}$

\section{Group sessions}

The $\mathrm{MCl}$ is offered as a group treatment to groups of 5 patients and additional family members and/or friends. The $\mathrm{MCl}$ will last 9 weeks. During the first 5 weeks, group sessions will take place once a week, followed by a booster session 3 weeks later. The group sessions last 2-2.5 hours and are led by an NP with experience in working with epilepsy patient groups. The first session is aimed at providing information about the $\mathrm{MCl}$, including materials, and getting to know the other participants and therapists. During the next sessions, participants will practice with the five stages of proactive coping defined by Aspinwall \& Taylor. ${ }^{30}$ These five stages are (1) resource accumulation, (2) recognition of potential stressors, (3) initial appraisal, (4) preliminary coping efforts, and (5) elicitation and use of feedback concerning initial efforts.

The model will be applied by the patients to three fixed epilepsy-related themes. The first theme will be self-monitoring and self-monitoring (e-Health) tools. The other two themes will be risk-management and shared decision-making/concordance.

Each group session will have the same basic structure. The sessions will begin by looking back at goals set in the previous meeting and how things have worked in the last week. Next, the theme of the session will be introduced. Patients and caregivers will be invited to share their beliefs, emotions and experiences with regard to the theme. Subsequently, patients and caregivers will formulate their own action plan in order to attain a goal relevant to the theme. Patients will be instructed to keep their goals feasible, and group members will give feedback on the quality of the goals in terms of concreteness and attainability. They will help each other to recognize additional conditions and barriers which need to be addressed. After the feedback discussion, patients and caregivers will formulate their final plan.

\section{EHealth}

The e-Health tools used in the intervention consist of 3 elements: 1) the Medication Event Monitoring System (MEMS; Aardex Ltd., Switzerland), 2) a smartphone application, and 3) an internet accessible patient database. The MEMS caps are electronic caps that fit on standard pill bottles. They register the date and time every time the pill bottle is opened. In addition, the MEMS of the intervention group will include an LCD-screen which 
provides feedback on the number of times the bottle is opened on a particular day. These data can be downloaded from the MEMS cap by the researchers with a communication device and a computer. A computer program will then present the data in simple plots which can be used to provide feedback about behaviour, and to identify sub-optimal adherence patterns. ${ }^{31}$ Feedback will be provided during the $\mathrm{MCl}$ and during each follow-up visit. The smartphone application ("Eppy"; Epilepsy Foundation, the Netherlands) is designed to register seizure frequency and other facts for persons with epilepsy, in order to provide data which can influence the management of epilepsy. "Eppy" can be downloaded at no charge from the App store (Apple Inc., USA) or from the Google Play store (Google Inc., USA). The application provides patients with the opportunity, among others, to keep a seizure diary, to set reminders for the intake of medicines and an alarm mode in which a text will be shown for bystanders in case of a seizure. Data gathered with "Eppy" can be synchronized to an internet accessible database, which gives an overview of all clinical events registered by the patient. This internet website is owned by the patient, who can allow healthcare professionals to access it for clinical and research purposes.

\section{Booster session}

During the booster session the NP will rehearse goal setting themes and other themes discussed in the group sessions. Patients and caregivers will have the opportunity to discuss their experiences related to their goals and other aspects of the intervention.

\section{Control group}

The control condition will be a control condition with unrestricted access to CAU. Care will not be intensified after enrolment. As this will be a pragmatic trial, CAU will not follow a standardized protocol. However, medical support will be documented in the electronic patient file of each patient. Medical support provided in the control group might be variable but is expected to be in agreement with the standard epilepsy guidelines (i.e. preference for mono-drug therapy, a monitoring visit every 4 months by neurologist or epilepsy nurse and change or addition of medication if the first AED is ineffective). ${ }^{32}$ The control group will receive MEMS but without feedback about their behaviour. The MEMS will be read only at the end of the follow-up as a way to measure adherence and will not include an LCD-screen.

\section{Clinical effectiveness}

To assess the (cost)-effectiveness of the $\mathrm{MCl}$, outcome assessments will be done at baseline and after 3, 6, 9, and 12 months (9 and 12 months applicable only for intervention group). A detailed overview regarding the outcome assessments can be found in Table 2.1. The following outcomes are defined: 
Table 2.1: Overview of measurements per time point

\begin{tabular}{|c|c|c|c|c|c|c|c|}
\hline Outcomes Patients & Instrument & Short term & TO & $\mathrm{T} 1$ & $\mathrm{~T} 2$ & $\mathrm{~T} 3 * *$ & $\mathrm{~T} 4 * *$ \\
\hline $\begin{array}{l}\text { Demographic and clinical } \\
\text { characteristics }\end{array}$ & & - & $x$ & - & - & - & - \\
\hline Self-efficacy & $\begin{array}{l}\text { Epilepsy Self-Efficacy Scale - } 33 \\
\text { items }\end{array}$ & ESES & $x$ & $x$ & $x$ & $x$ & $x$ \\
\hline General Self-efficacy & $\begin{array}{l}\text { Generic Self-Efficacy Scale - } 10 \\
\text { items }\end{array}$ & GSES & $x$ & $x$ & $x$ & $x$ & $x$ \\
\hline Adherence & $\begin{array}{l}\text { MEMS } \\
\text { Medication Adherence Scale }\end{array}$ & MARS 5 & $x$ & $x$ & $x$ & $x$ & $x$ \\
\hline Seizure frequency & Questionnaire seizure frequency & - & $x$ & $x$ & $x$ & $x$ & $x$ \\
\hline Seizure severity & $\begin{array}{l}\text { National Hospital Seizure Severity } \\
\text { Scale }\end{array}$ & NHS3 & $x$ & $x$ & $x$ & $x$ & $x$ \\
\hline Emotional functioning & $\begin{array}{l}\text { Hospital Anxiety and Depression } \\
\text { Scale }\end{array}$ & HADS & $x$ & $x$ & $x$ & $x$ & $x$ \\
\hline \multirow[t]{2}{*}{ Quality of life } & \multirow{2}{*}{$\begin{array}{l}\text { Quality Of Life in Epilepsy } \\
\text { Generic quality of life }\end{array}$} & \multirow{2}{*}{$\begin{array}{l}\text { QOLIE31P } \\
\text { EQ-5D-5L }\end{array}$} & $x$ & $x$ & $x$ & $x$ & $x$ \\
\hline & & & $x$ & $x$ & $x$ & $x$ & $x$ \\
\hline Proactive Coping & $\begin{array}{l}\text { Utrecht Proactive Coping } \\
\text { Competence }\end{array}$ & UPCC/PCI & $x$ & $x$ & $x$ & $x$ & $x$ \\
\hline Side Effect & Side effects of Anti-Epileptic Drugs & SIDAED & $x$ & $x$ & $x$ & $x$ & $x$ \\
\hline Healthcare resource use & $\begin{array}{l}\text { Medical Cost Questionnaire } \\
\text { Productivity Cost Questionnaire }\end{array}$ & $\begin{array}{l}\mathrm{MCQ} \\
\mathrm{PCQ}\end{array}$ & $x$ & $x$ & $x$ & $x$ & $x$ \\
\hline
\end{tabular}

*T0 = baseline outcome assessments; T1=Outcome assessments after 3 months; T2=Outcome assessments after 6 months; T3=Outcome assessments after 9 months; T4=Outcome assessments after 12 months; ** Outcome assessment applicable only for intervention group

\section{Primary outcomes}

Epilepsy Self-Efficacy Scale-33 items (ESES). The ESES is a 33-item scale that measures different aspects of efficacy within the self-management of epilepsy. The items represent three dimensions of self-management: medication management, seizure management, and general management including safety and health. ${ }^{25}$ Items are rated on an 11-point Likert rating scale, ranging from 0 , "not at all certain I can do", to 10, "very certain I can do". ${ }^{33}$ The total possible scores for the ESES range from 0 to 330. Higher scores correspond to higher levels of confidence in the ability to manage epilepsy.

\section{Secondary outcomes}

Adherence, used as a proxy for concordance, which will be determined using: 1) MEMS. Electronic monitoring, such as MEMS has been proposed as a possible "gold standard" for medication adherence measurement ${ }^{15,34}$, and 2) the Medication Adherence Rating Scale (MARS-5), which measures self-reported adherence. The MARS-5 contains 10 items, each of which has to be answered with yes or no. Hence, the final score on the MARS-5 ranges from 0 to 10, in which a higher score represents better adherence. 
General self-efficacy, which will be determined using the Dutch adaptation of the general Self-Efficacy Scale (GSES). The GSES consists of 10 items assessed on a 4-point scale, ranging from 'totally wrong' to 'totally true'. The scale was designed to assess self-efficacy, i.e., the belief that one's actions are responsible for successful outcomes. The scale scores for each question ranges from 1 to 4 resulting in an overall score between 10 and 40. Higher scores indicate patient's stronger belief in self-efficacy. ${ }^{35,36}$

Seizure frequency will be determined using a short questionnaire regarding seizure frequency covering the past 4 weeks. The questions focus mainly on the number of seizures, whether the person documents his/her seizures and in what way the person documents his/her seizures.

Seizure severity, which will be determined using the National Hospital Seizure Severity Scale (NHS3). The NHS3 lists seven seizure-related factors and generates a score from 1 to 27 , in which a higher score represents a more severe seizure. ${ }^{37}$

Adverse events of AED, which will be determined using the SIDe-effect of the AntiEpileptic Drugs questionnaire (SIDAED). The SIDAED consists of 46 items regarding possible AED-related complaints. These items form 10 categories: general CNS, behaviour (increased irritability), depressive symptoms, cognitive function, motor problems and coordination, visual complaints, headache, cosmetic and dermatological complaints, gastrointestinal complaints, and sexuality and menses. ${ }^{38}$ For each item the patient rates the severity of the complaint on a four- point Likert scale (no problem, mild, moderate, or serious problem). In addition, the duration of the complaints is scored (a few weeks, months or half a year or longer). The SIDAED ranges from 0 to 138, in which a higher score indicates more severe/frequent side-effects. ${ }^{38}$

Depression/Anxiety, which will be determined using the Hospital Anxiety and Depression Scale (HADS). The HADS has a total of 14 items, each scored on a scale of 0-3, with 3 indicating higher symptom frequencies. Scores for each subscale (anxiety and depression) can range from 0-21 and scores for the entire scale (emotional distress) range from $0-42$, with higher scores indicating more distress. ${ }^{39}$

Self-rated proactive coping, which will be determined using the Utrecht Proactive Coping Competence Scale (UPPC). A total of 21 items are assessed on a 4-point scale ranging from 'not competent at all' to 'very competent'. Total scores are calculated by averaging the 99 individual item scores. Higher scores on the UPCC indicate higher levels of perceived proactive coping competencies. ${ }^{40}$

Disease-specific quality of life will be measured with the Quality Of Life in Epilepsypatient-weighted (QOLIE-31-P). The QOLIE-31-P consists of 38 items assessing 7 domains of epilepsy: Seizure Worry, Overall QOL, Emotional well-being, Energy-Fatigue, Cognitive Functioning, Medication Effects, Social Functioning and an Overall Score. In addition, for each domain, questions regarding how much distress a person feels about problems and worries related to epilepsy are included. ${ }^{41}$ Each domain is scored on a scale ranging from 0 to 100 . Afterwards a final score can be calculated using weights derived from the 
amount of distress related to each domain. The final score ranges between 0 to 100, in which higher values indicate a better Quality of Life. ${ }^{42}$

Generic Quality of Life will be assessed with the EuroQoL 5 dimensions 5 levels (EQ5D-5L). The EQ-5D-5L consists of five dimensions: mobility, self-care, usual activities, pain/discomfort, anxiety/depression, each of which can have one of five responses. ${ }^{43,44}$ Each health state will be valued using the Dutch tariffs, which will result in utilities on a scale from 0 to 1 . Utilities derived from the EQ-5D-5L will be used in calculating the quality adjusted life years (QALY) by multiplying the time spent in a health state by the utility assigned to that health state.

Societal costs will be measured retrospectively with the Medical Cost Questionnaire (MCQ), an adapted version of the Trimbos/iMTA questionnaire for costs associated with psychiatric illness ${ }^{45}$ and the Productivity Cost Questionnaire (PCQ), each covering 3 months.

\section{Economic evaluation}

The trial-based economic evaluation will be performed from a societal perspective and will consist of a cost-effectiveness analysis (CEA) and a cost-utility analysis (CUA). Outcomes of interest for the CEA and the CUA will be self-efficacy as assessed by the ESES, and generic quality of life as assessed by EQ-5D-5L. We distinguish four cost categories: intervention costs, healthcare sector costs, costs for the patient and family, and productivity costs. Intervention costs will be defined as all costs related to the $\mathrm{MCl}$ including travel costs, personal costs, material costs, costs of e-Health tools (i.e. MEMS) and housing costs. Healthcare and patient costs will be estimated using a questionnaire regarding healthcare resource utilization and productivity losses.

Resource use and outcomes are measured at the same time points mentioned in the effectiveness study: at baseline, 3 months, 6 months, 9 months, and 12 months in the intervention group and at 3 months and 6 months in the control group. A comparison between $\mathrm{MCl}$ and $\mathrm{CAU}$ will be made in terms of incremental costs and incremental effects. The time horizon will be 12 months (trial-based economic evaluation). To measure the use of health care resources, including all activities related to epilepsy, we gather data for each patient at baseline and at a follow-up of one year. Cost calculations will be based on the Dutch guidelines for cost calculations in healthcare. ${ }^{46}$

\section{Process evaluation}

Process evaluation will be performed to assess whether the $\mathrm{MCl}$ was delivered according to protocol, to examine the experiences and opinions of patients, caregivers and professionals regarding the $\mathrm{MCl}$, and to determine to what extent the $\mathrm{MCl}$ has impacted concordance among patients. The process evaluation will be performed according to the framework provided by Saunders et al. ${ }^{47}$ This framework consists of a stepwise approach in which important characteristics for the process-evaluation plan are identified along 
seven basic components, namely: fidelity (quality), dose delivered (completeness), dose received (exposure), dose received (satisfaction), reach (participation rate), recruitment and context.

We will use a mixed methods design in which both qualitative and quantitative data will be collected. The qualitative part will consist of observations during several group sessions over time. After each observation, a short interview will be held with the group leader(s) in which the group leader can reflect on his/her opinion regarding the session. During the last (sixth) group session of every $\mathrm{MCl}$-group, a short evaluation form will be handed out to patients in which they will be asked to rate different aspects and themes of the $\mathrm{MCl}$ on a 7-point Likert scale. Furthermore, at the end of the study, focus groups will be held consisting of patients included in the study. The selection of participants will be based on maximal variation to get as many perspectives as possible. Participants will be selected based on age, sex and severity of seizures and effectiveness of the intervention (to compare patients for which the intervention was successful versus unsuccessful). Focus groups will be conducted using a semi-structured questionnaire covering the topics identified in the framework provided by Saunders et al. ${ }^{47}$

\section{ANALYSIS}

\section{Clinical effectiveness}

Baseline characteristics will be described, and differences between groups at baseline will be studied using t-tests or chi-square tests where appropriate. All statistical procedures will be conducted based on both the intention-to-treat principle and on actual participation in treatment (i.e. effectiveness analyses) and will be performed using SPSS statistics 22.0 (SPSS, IBM, Corporation, Chicago, USA). Missing data will be handled using SPSS missing value analysis on item level. Completely missing measurements will be handled using multiple imputation. To evaluate outcomes, change scores will be calculated and compared between the groups after treatment. Multi-level analyses will be performed with measurements (T0, T1, T2, T3 and T4) within the subjects' factor and group and between subjects' factors to account for the nested structure of the data. Data from the control group will be extrapolated to 12 months. Post hoc analyses will be performed in case of significant effects. Baseline differences will be corrected by inclusion of covariates in the analyses. A 2-sided significance level of 0.05 will be used as a threshold to determine whether differences are statistically significant.

\section{Economic evaluation}

Costs calculation will be performed according to the bottom-up approach, based on a detailed inventory of all cost items. Standardized cost prices from the Dutch manual for costing will be used in the calculations or (if not available) calculated mean cost prices, 
according to providers, will be used. ${ }^{46}$ To determine the costs of drugs, the Dutch consumer reimbursement price for medication will be used. Productivity costs of the patients will be estimated with the friction cost method. Costs will be indexed for the year 2015 .

As cost data are normally skewed, parametric tests are mostly not suitable. Hence non-parametric bootstrapping (1000 times) will be used to test for statistical differences in costs between groups and to investigate the uncertainty around the costs. Bootstrapping will be done using Microsoft Excel (Excel, Microsoft Corporation, Washington, USA). Bootstrap replications will be used to calculate 95\% confidence intervals around the costs, based on the 2.5th and 97.5th percentiles. For the cost-effectiveness analysis, the corresponding incremental cost-effectiveness ratio (ICER) will be expressed as incremental costs per increased adherence and incremental costs per self-efficacy (ESES). In the cost-utility analysis, the ICER will be expressed as the incremental costs per QALY gained. QALYs will be calculated using the area under the curve method. All bootstrapped ICERs (5000 times) will be presented in a cost-effectiveness plane to determine the robustness of the ICER. To determine the probability that the $\mathrm{MCl}$ is cost-effective given a certain ceiling ratio, a cost-effectiveness acceptability curve will be constructed. In addition, oneway and multi-way sensitivity analysis will be performed on the most important cost parameters.

\section{Process evaluation}

Quantitative data will be analysed by descriptive statistics (i.e. frequencies, mean and median), Chi square tests, and ANOVA. Results from open-ended questions included in the questionnaires, focus group interviews and interviews will be categorized to identify relevant themes.

\section{DISCUSSION}

This study will determine the (cost-)effectiveness and feasibility of the $\mathrm{MCl}$ to improve the management of epilepsy in adult patients and increase self-efficacy and concordance regarding AED. The $\mathrm{MCl}$ is designed to stimulate self-management skills and the awareness of patients with epilepsy in combination with the use of e-Health interventions. In times of budget constraints, $\mathrm{MCl}$ could be a valuable addition to current healthcare provisions for epilepsy, as it is expected that higher concordance and self-efficacy will result in reduced healthcare resource use and an increased QOL.

Cooperation between research and practice is the key strength of this project, enabling the intervention to be studied in a natural environment; the project will facilitate further implementation of the multi-component program into the standard practice of $\mathrm{KH}$ and of other institutions. Both professionals and patients played important roles in 
the development of this program and will also be involved during the evaluation and implementation of the $\mathrm{MCl}$ program.

One of the limitations of our study design is that, in case the $\mathrm{MCl}$ is shown to be effective, it will be difficult to identify what components contribute to this effectiveness. For example, it could be that only the increased attention from nurses or the adherence monitoring, contribute to improvements in the patients' health status. However, we believe we have included a large variety of outcome assessments which, altogether, form a broad view of the (possible) effectiveness of $\mathrm{MCl}$.

To the best of our knowledge, there are currently no validated questionnaires available to examine concordance. However, as this study is striving to improve 'concordance' and not only 'adherence', it is assumed that any increase in adherence by the $\mathrm{MCl}$ is partly explained by increased concordance (i.e. due to the educational content in the $\mathrm{MCl}$ ). In addition, special attention will be paid to concordance during the process evaluation. Accordingly, this study is aimed at making patients their own provider of health care, thus shifting epilepsy management from professionals to self-care by patients equipped with appropriate skills and tools. 


\section{REFERENCES}

1. Fact sheet Epilepsy. In: World Health Organisation; http://www.who.int/mediacentre/factsheets/fs999/ en/index.html ; 2012.

2. Jacoby A, Baker GA. Quality-of-life trajectories in epilepsy: a review of the literature. Epilepsy Behav 2008; 12: 557-71.

3. Taylor RS, Sander JW, Taylor RJ, Baker GA. Predictors of health-related quality of life and costs in adults with epilepsy: a systematic review. Epilepsia 2011; 52: 2168-80.

4. Kotsopoulos IA, Evers SM, Ament AJ, de Krom MC. Estimating the costs of epilepsy: an international comparison of epilepsy cost studies. Epilepsia 2001; 42: 634-40.

5. Kotsopoulos IA, Evers SM, Ament AJ, Kessels FG, de Krom MC, Twellaar M, et al. The costs of epilepsy in three different populations of patients with epilepsy. Epilepsy Res 2003; 54: 131-40.

6. Elwes RD, Marshall J, Beattie A, Newman PK. Epilepsy and employment. A community based survey in an area of high unemployment. J Neurol Neurosurg Psychiatry 1991; 54: 200-3.

7. Hart YM, Shorvon SD. The nature of epilepsy in the general population. I. Characteristics of patients receiving medication for epilepsy. Epilepsy Res 1995; 21: 43-9.

8. Pato Pato A, Cebrian Perez E, Cimas Hernando I, Lorenzo Gonzalez JR, Rodriguez Constenla I, Gude Sampedro F. Analysis of direct, indirect, and intangible costs of epilepsy. Neurologia 2011; 26: 32-8.

9. Strzelczyk A, Reese JP, Dodel R, Hamer HM. Cost of epilepsy: a systematic review. Pharmacoeconomics 2008; 26: 463-76.

10. Loiseau J, Picot M, Loiseau P. Prognosis of epileptic syndromes: EPIGIR study. Prognosis of Epilepsies. Montrouge: John Libbey 2003: 29-38.

11. Kwan P, Brodie MJ. Early identification of refractory epilepsy. N Engl J Med 2000;342: 314-9.

12. Wallace $H$, Shorvon S, Tallis R. Age-specific incidence and prevalence rates of treated epilepsy in an unselected population of 2,052,922 and age-specific fertility rates of women with epilepsy. Lancet 1998; 352 : 1970-3.

13. Haynes RB, Ackloo E, Sahota N, McDonald HP, Yao X. Interventions for enhancing medication adherence. Cochrane Database Syst Rev 2008: CD000011.

14. Oberje EJ, de Kinderen RJ, Evers SM, van Woerkum CM, de Bruin M. Cost effectiveness of medication adherence-enhancing interventions: a systematic review of trial-based economic evaluations. Pharmacoeconomics 2013; 31: 1155-68.

15. Jones RM, Butler JA, Thomas VA, Peveler RC, Prevett M. Adherence to treatment in patients with epilepsy: associations with seizure control and illness beliefs. Seizure 2006; 15: 504-8.

16. Cramer JA, Roy A, Burrell A, Fairchild CJ, Fuldeore MJ, Ollendorf DA, et al. Medication compliance and persistence: terminology and definitions. Value Health 2008; 11: 44-7.

17. Amir M, Roziner I, Knoll A, Neufeld MY. Self-Efficacy and Social Support as Mediators in the Relation Between Disease Severity and Quality of Life in Patients with Epilepsy. Epilepsia $\quad$ 1999;40: 216-224.

18. Bodenheimer T, Lorig K, Holman H, Grumbach K. Patient self-management of chronic disease in primary care. JAMA 2002; 288: 2469-75.

19. Pramuka M, Hendrickson R, Zinski A, Van Cott AC. A psychosocial self-management program for epilepsy: a randomized pilot study in adults. Epilepsy Behav 2007; 11: 533-45.

20. Samsonsen C, Reimers A, Brathen G, Helde G, Brodtkorb E. Nonadherence to treatment causing acute hospitalizations in people with epilepsy: an observational, prospective study. Epilepsia 2014; 55: e125-8.

21. Lorig K, Chastain RL, Ung E, Shoor S, Holman HR. Development and evaluation of a scale to measure perceived self-efficacy in people with arthritis. Arthritis Rheum 1989; 32: 37-44.

22. Lorig K, Gonzalez VM, Ritter P. Community-based Spanish language arthritis education program: a randomized trial. Med Care 1999; 37: 957-63.

23. Lorig KR, Holman H. Self-management education: history, definition, outcomes, and mechanisms. Ann Behav Med 2003; 26: 1-7. 
24. Shegog R, Bamps YA, Patel A, Kakacek J, Escoffery C, Johnson EK, et al. Managing Epilepsy Well: Emerging e-Tools for epilepsy self-management. Epilepsy Behav 2013; 29: 133-40.

25. Dilorio C, Yeager K. The Epilepsy Self-Efficacy Scale. In: C SOD, editor. Measurement of nursing outcomes: Self care and coping, 2nd ed. New York: Springer Publishing Company; 2003, p. 40-51.

26. Ouwens M, van der Brug S, Faber M, van der Weijden T. Shared Decision Making \& Zelfmanagement. In. Nijmegen: Scientific Institute for Quality of Healthcare; 2012.

27. van Eeden M, van Heugten C, Evers SM. The economic impact of stroke in The Netherlands: the E-restore4stroke study. BMC Public Health 2012;12: 122.

28. Miller W, Rollnick S. Ten things that motivational interviewing is not. Behavioural and Cognitive Psychotherapy 2009; 37: 129.

29. Miller W, Rollnick S. Motivational interviewing: Preparing people for change: Guilford press; 2002.

30. Aspinwall LG, Taylor SE. A stitch in time: self-regulation and proactive coping. Psychol Bull 1997; 121: 41736.

31. Data collection In: MWV Aardex; http://www.mwvaardex.com/Products/Data Collection/index.htm; 2014.

32. Nederlandse Vereniging voor Neurologie Commissie Kwaliteitsbevordering. Richtlijnen diagnostiek en behandeling van Epilepsie. Herziene, tweede versie: januari 2006.

33. Dilorio C, Faherty B, Manteuffel B. The development and testing of an instrument to measure self-efficacy in individuals with epilepsy. J Neurosci Nurs 1992; 24: 9-13.

34. Bertholet N, Favrat B, Fallab-Stubi CL, Brunner HR, Burnier M. Why Objective Monitoring of Compliance is Important in the Management of Hypertension. J Clin Hypertens (Greenwich) 2000; 2: 258-262.

35. Sherer M, Maddux JE, Mercandante B, Prentice-Dunn S, Jacobs B, Rogers RW. The self-efficacy scale: Construction and validation. Psychological reports 1982; 51: 663-671.

36. Teeuw B, Schwarzer R, Jerusalem M. Dutch adaptation of the general self-efficacy scale. Berlin, Germany 1994.

37. O'Donoghue MF, Duncan JS, Sander JWAS. The National Hospital Seizure Severity Scale: A Further Development of the Chalfont Seizure Severity Scale. Epilepsia 1996; 37: 563-571.

38. Uijl SG, Uiterwaal CS, Aldenkamp AP, Carpay JA, Doelman JC, Keizer K, et al. A cross-sectional study of subjective complaints in patients with epilepsy who seem to be well-controlled with anti-epileptic drugs. Seizure 2006; 15: 242-8.

39. Zigmond AS, Snaith RP. The hospital anxiety and depression scale. Acta Psychiatr Scand 1983; 67: 361-70.

40. Tielemans NS, Visser-Meily JM, Schepers VP, Post MW, van Heugten CM. Proactive coping poststroke: psychometric properties of the Utrecht Proactive Coping Competence Scale. Arch Phys Med Rehabil 2014; 95: 670-5.

41. Devinsky O, Vickrey BG, Cramer J, Perrine K, Hermann B, Meador K, et al. Development of the quality of life in epilepsy inventory. Epilepsia 1995; 36: 1089-104.

42. QOLIE-31-PC: Cramer JA, Van Hammee, G., N132 Study Group. Maintenance of improvement in healthrelated quality of life during long-term treatment with levetiracetam. Epilepsy \& Behavior 2003; 4:118123.

43. Brooks R. EuroQol: the current state of play. Health Policy 1996; 37: 53-72.

44. Dolan P. Modeling valuations for EuroQol health states. Med Care 1997; 35: 1095-108.

45. Hakkaart-van Roijen L, Straten Av, Tiemens B, Donker M. Handleiding Trimbos/iMTA questionnaire for Costs associated with Psychiatric illness (TiC-P). In: Institute of Medical Technology Assessment (iMTA); 2002.

46. Hakkaart-van Roijen L, Tan S, Bouwmans C. Handleiding voor kostenonderzoek. Methoden en standaard kostprijzen voor economische evaluaties in de gezondheidszorg. Geactualiseerde versie 2010.

47. Saunders RP, Evans MH, Joshi P. Developing a process-evaluation plan for assessing health promotion program implementation: a how-to guide. Health Promot Pract 2005; 6: 134-47. 



\section{CHAPTER}

\section{Are People with Epilepsy using eHealth-tools?}

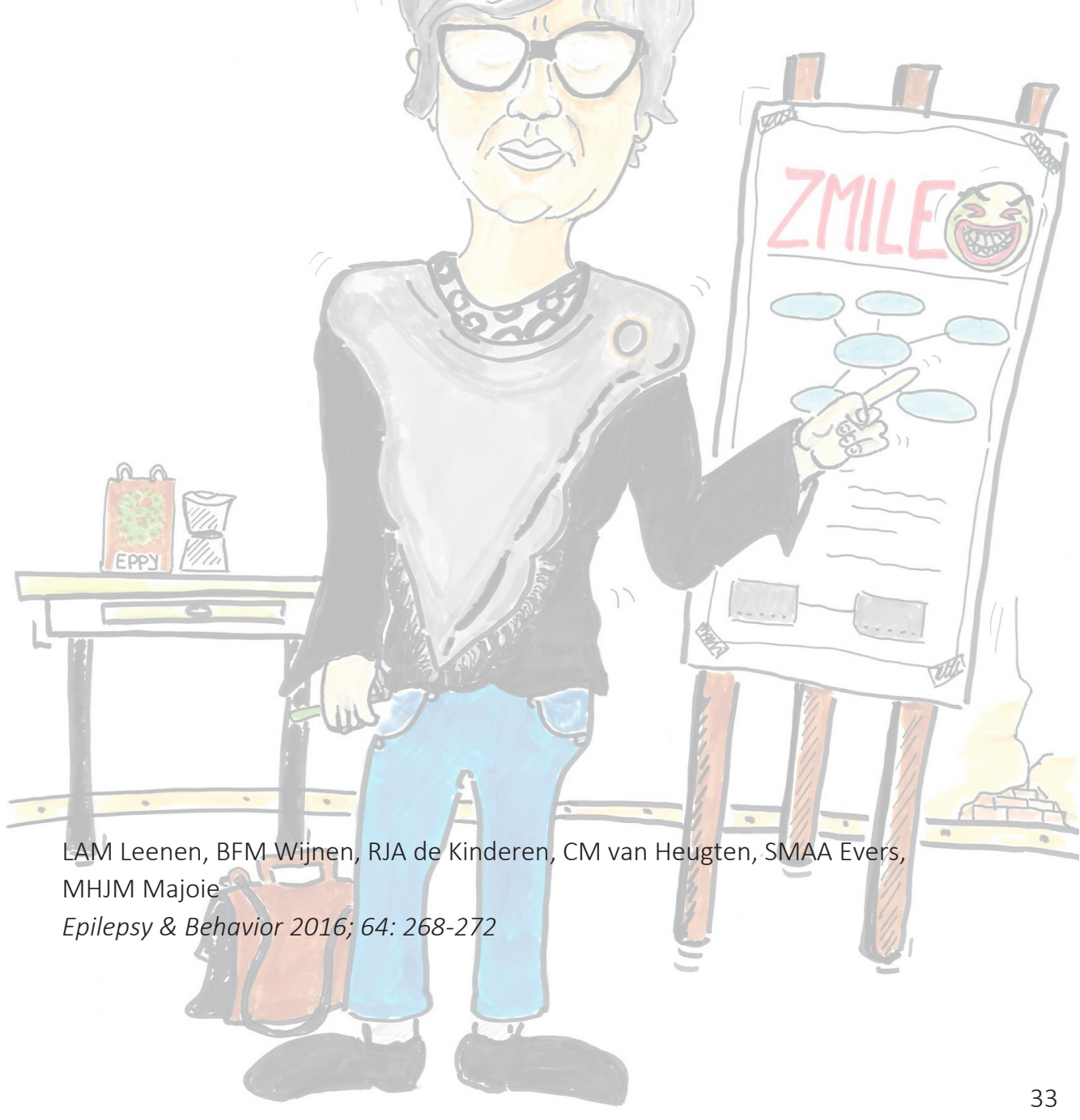




\section{ABSTRACT}

\section{Introduction}

Self-management for people with epilepsy (PWE) should lead to shared decision-making and thus to adherence to the treatment plan. EHealth is an important way of supporting PWE in their self-management.

\section{Method}

In this survey, we used a mixed method to explore: 1) which factors were monitored by PWE, and how (using pen and paper or eHealth-tools), 2) how many PWE own a computer or smartphone, and 3) how they perceive the use of eHealth. A consecutive series of 1000 PWE attending the outpatient clinic of a tertiary epilepsy centre were asked to fill in a questionnaire.

\section{Results}

In comparison with the general population, fewer PWE owned a computer or smartphone. They are, however, more likely to self-monitor their health than other patients suffering from a chronic condition. Although PWE do not use eHealth-tools often, they perceive it as a user-friendly tool, promoting health behaviour as well as adherence. On the other hand, problems with privacy and the perception that not everyone is able to use eHealth, are considered as disadvantages by PWE. Promoting self-care was perceived as both an advantage and a disadvantage. It was seen as an advantage when PWE mention the option of eHealth-tools in order to gain insight into one's epilepsy. At the same time, it was seen as a disadvantage because it confronts PWE with their disease, which causes emotional stress.

\section{Conclusion}

The high level of self-monitoring combined with a low usage of eHealth-tools seems to indicate that there is a need for a more tailored approach to stimulate the use of eHealthtools by PWE. Further research should focus on this aspect, e.g. what do PWE need in order to make more use of eHealth-tools in their self-care. 


\section{INTRODUCTION}

Epilepsy is a disorder of the brain, characterized by recurrent seizures. Seizures are the result of sudden electrical discharges in groups of brain cells. These discharges result in a variety of clinical manifestations, depending on where they occur in the brain. ${ }^{1}$

For the treatment of people with a chronic condition, such as epilepsy, self-management is believed to be an important factor in sustaining or increasing quality of life and quality of care. ${ }^{2}$ Self-management refers to the ability of patients to cope with their chronic condition, eventually aiming to maximize quality of life. ${ }^{3}$

In order to manage their life, people with epilepsy (PWE) have to become knowledgeable about their disease, and the factors influencing the disease and treatment. Furthermore, they have to adhere to treatment and lifestyle regimens and to cope with the psychosocial consequences of having this chronic condition. ${ }^{4,5}$

The tools to support self-management for people with a chronic condition range from individual and group training sessions to (smart-)phone applications (often regarded as "apps") and websites. ${ }^{6}$ During the past decade, the latter two forms of delivering information has become more popular as shown by the increasing amount of online information, online health services and online self-help programs, often regarded as eHealth. EHealth is defined as the use of information communication technologies (ICT) to improve or enable health and healthcare. ${ }^{6}$ It often incorporates wireless, mobile electronic tools (eHealth-tools). ${ }^{6}$

In order to support individuals with a chronic condition in their self-management, eHealth-tools need to be more than merely a source of information. ${ }^{7,8}$ Some examples of tools that offer more are the e-medication feedback tool ${ }^{9}$ and e-diaries. ${ }^{10}$ Both applications have been shown to improve adherence.

EHealth is an easily accessible and convenient way of reaching a large target group and, more importantly, potentially leading to improved health outcomes. ${ }^{11,12}$ For this reason, the Dutch government has incorporated the development and application of eHealth in their objectives to establish quality of health care. ${ }^{13}$ In addition, the Dutch government is trying to use eHealth as a way of controlling health costs in an aging society. ${ }^{2}$ In 2015 , the government stated that within five years, $80 \%$ of the patients with a chronic condition should have access to their medical records, preferably using mobile- or internet-based applications. Seventy-five percent of these patients should have the option and be able to self-monitor their condition using eHealth. ${ }^{8}$ The proportion of people with a chronic condition already monitoring their disorder in 2015 was 40\%. Ten percent were using a computer and $3 \%$ a smartphone.

Currently, several eHealth-tools are available for PWE, such as "My seizure diary" (www. Myseizurediary.com) and "Seizure tracker" (www.seizuretracker). These eHealthtools are designed so that PWE can register seizure frequency and other epilepsy-related factors, in order to provide data that can support the management of epilepsy. ${ }^{14}$ Previous studies have shown that eHealth is able to improve health outcomes in other chronic 
conditions $^{15,16}$, including epilepsy ${ }^{17}$, this study showed an improvement in self-management and self-efficacy outcomes of an online self-management programme.

It has been shown, however, that not every group of patients with a chronic condition benefits equally from the growing body of eHealth-tools. ${ }^{18,19}$ This is due to several barriers to using eHealth-tools to their full potential, such as lack of access to hardware (e.g. personal computer or laptop, smartphone or tablet) and the lack of skills required to use eHealth-tools properly. ${ }^{20,21}$

In a recent study by the US Centre for Disease Control, the authors suggested that the use of easy-to-access resources and eHealth-tools might help PWE to optimize their selfmanagement and eventually improve their quality of life. ${ }^{22}$ There is, however, no body of evidence regarding the effectiveness of eHealth-tools for PWE.

The main purpose of this paper was, therefore, to explore whether PWE have access to hardware and if they perceive eHealth-tools as useable in their (self)-care.

This study focuses on three main topics:

1. Which events are self-monitored and which tools do PWE use?

2. In comparison to the general Dutch population, how many PWE possess hardware that can be used for eHealth?

3. What are the advantages and disadvantages of eHealth as perceived by PWE - both users and non-users of eHealth-tools?

\section{Methods}

\section{Population}

This study was conducted among patients diagnosed with epilepsy, treated at Kempenhaeghe, a tertiary epilepsy centre serving the southern part of The Netherlands.

A consecutive series of 1,000 patients who visited the Kempenhaeghe outpatient clinic in the first trimester of 2013 were contacted to participate in the study in order to obtain a representative sample. Respondents were either adults with epilepsy or parents/caregivers serving as proxy for their child with epilepsy and/or for people with cognitive deficits due to their epilepsy. In the case of the latter respondents, the cover letter was addressed to the parents or carers.

\section{Procedure and survey}

The survey was conducted using a self-completion 14-item questionnaire (Appendix 3.1), sent with a cover letter explaining the reasons for the study and asking the respondent or parent(s) by proxy to fill it in. After 8 weeks, a reminder was sent. Respondents consented to participate by returning the questionnaire. The local medical ethics review board of the Centre for Epilepsy Kempenhaeghe approved this study.

The survey covers the following topics: 
- Demographic information; including age, gender and who completed the questionnaire.

- Use and content of self-monitoring tool; which events were self-monitored, the reason for self-monitoring these events, and whether respondents used eHealth-tools or a more traditional way (e.g. using pencil and paper) of collecting data.

- Possession of hardware; whether or not respondents owned hardware such as a computer, smartphone or tablet.

- Perceived advantages and disadvantages of eHealth: two open-ended questions.

\section{ANALYSIS}

All analyses were conducted in SPSS Version 21. Descriptive statistics were used to summarize important variables. Pearson Chi square test was used to determine statistical differences between binary variables (e.g. gender), and logistic regression to determine the effect of categorical variables (i.e. 4 age groups: $12-24 y ; 25-44 y ; 45-64 y$ and $65-100 y$ ) on binary outcome measures (i.e. possession of; smartphone $y / n$, computer $y / n$ and tablet $y / n)$. Age groups were defined according to the categorization of the Statistics Netherlands. ${ }^{23}$

Analyses of the open-ended questions were carried out using a qualitative method. The answers to these open-ended questions were labelled according to the description used in the systematic review of Interactive Health applications for people with chronic conditions by Murray et al. ${ }^{7}$ The following categories were defined: 1) relaying information; 2) enabling shared decision-making; 3) promoting health behaviours e.g. using medication reminders or alarms; 4) promoting peer information exchange and support; 5) promoting self-care e.g. using a seizure diary and scoring seizure-provoking factors; 7) managing demands for health services; 8 ) equity (equity is the accessibility by means of eHealth to services, health care and health outcome); 9) quality criteria such as privacy etc.; 10) other (this category was added to accommodate the responses that could not be assigned to one of the above categories). In addition to this framework, user-friendliness was added as an extra category (11), because it was frequently mentioned by the respondents.

\section{RESULTS}

A total of 571 respondents (57\%) completed the questionnaire, 284 of whom were female (50.7\%). In the case of 11 respondents, gender was not recorded.

The mean age was 38.3 years (SD $=18.5$ years), The survey was completed by PWE ( $\mathrm{n}$ = 259), in 8 cases by the partners and in 175 instances by parents/caregivers; for 129 surveys, it was not known who completed the questionnaire. 


\section{Use and content of self-monitoring tools}

Almost two-thirds (62.2\%) of the respondents monitor their seizures in some way, and approximately half of the respondents (52.7\%) used a kind of memory aid for taking antiepileptic drugs, such as a medication box (18\%) or alarm on their phone (14\%).

Eleven percent of the respondents have downloaded a health app (on a mobile device) in order to facilitate their self-monitoring.

As presented in Table 3.1, more than half of the respondents $(n=272)$ monitor other factors as well. Change of medication (66\%), side-effects (43\%), and the use of (36\%) emergency medication were the most mentioned medication related factors. Several other factors were also monitored, for instance stress factors (38\%), diurnal rhythm (19\%) and menstrual cycle (17\%).

Table 3.1: Overview of events self-monitored by respondents

\begin{tabular}{ll}
\hline Events self-monitored N (\%) & \\
\hline Seizure frequency & $354(62 \%)$ \\
Medication intake & $297(52 \%)$ \\
Other events monitored & $272(48 \%)$ \\
Medication-related events & $179(66 \%)$ \\
\hline Medication change & $118(43 \%)$ \\
Side-effects & $100(36 \%)$ \\
Frequency of using emergency medication & $65(24 \%)$ \\
Medication used for emergency reasons & $81(31 \%)$ \\
Forgotten AED intake & $10(4 \%)$ \\
Intake of contraceptives & $102(38 \%)$ \\
Distribution of other monitored events & $53(19 \%)$ \\
\hline Stress factors & $45(17 \%)$ \\
Diurnal rhythm & $16(6 \%)$ \\
Menstrual cycle &
\end{tabular}

Total $>100 \%$, because respondents could monitor more than one event.

The three most important reasons respondents indicated for self-monitoring were because: 1 ) it provides insight into their seizure pattern (91.3\%); 2) it helps to find factors influencing their seizures (87.6\%), and 3 ) they had been being asked by a neurologist (87.3\%).

Table 3.2 lists the tools used to self-monitor seizure frequency; i.e. this is usually a paper diary $(43 \%)$ rather than an eHealth-tool, such as a digital diary (16\%). With regard to an aid to assist medication intake, the traditional tools, such as a medication box (52\%) are used more often compared to eHealth-tools (14\%).

Possession of hardware.

Of the respondents, $82 \%$ owns a computer and 39\% a smartphone. No significant 
differences were found between males and females regarding access to any of these eHealth-tools.

Patients aged 12-24 did not significantly differ from patients aged 25-44 regarding the use of a personal computer (PC) or laptop, smartphone or tablet. However, patients aged 45-64 and patients aged over 65 used a pc or laptop $(p=.006 ; p=.000)$; smartphone $(p=$ $.000 ; p=.000)$ or a tablet $(p=.012 ; p=.006)$ significantly less often.

Table 3.2: Use of tools for self-monitoring and taking medication by people with epilepsy

\begin{tabular}{|c|c|c|c|c|}
\hline \multicolumn{2}{|c|}{ Tools to self-monitor seizure frequency } & \multirow[t]{2}{*}{$\mathrm{N}(\%)$} & \multicolumn{2}{|l|}{$\underline{\text { Tools to assist in taking medication }}$} \\
\hline \multicolumn{2}{|l|}{ eHealth-tool } & & \multicolumn{2}{|l|}{ eHealth-tool } \\
\hline Digital diary & $93(16 \%)$ & & Alert on phone & $79(14 \%)$ \\
\hline \multicolumn{2}{|l|}{ Other tools } & & \multicolumn{2}{|l|}{ Other aids } \\
\hline \multirow[t]{3}{*}{ Paper diary } & \multirow[t]{3}{*}{$247(43 \%)$} & & Medication box & $103(18 \%)$ \\
\hline & & & Drug dosage package & $45(8 \%)$ \\
\hline & & & Notification by family or carers & $55(10 \%)$ \\
\hline Other & $16(3 \%)$ & & Other & $21(5 \%)$ \\
\hline Missing & $24(5 \%)$ & & Missing & $24(4 \%)$ \\
\hline None & $194(33 \%)$ & & None & $244(43 \%)$ \\
\hline \multicolumn{3}{|c|}{$\begin{array}{l}\text { Other stands for: family or carers }(n=9) \text {; } \\
\text { camera }(n=2) \text { and memory }(n=5)\end{array}$} & \multicolumn{2}{|c|}{$\begin{array}{l}\text { Other stands for: alarm watches/clocks }(n=7) \text {, daily } \\
\text { routine }(n=12) \text {, visual aid }(n=2)\end{array}$} \\
\hline
\end{tabular}

\section{Advantages and/or disadvantages of using eHealth-tools}

One hundred sixty-six respondents mentioned one or two advantages; disadvantages were mentioned by 138 respondents. Some factors were mentioned as an advantage by part of the group whilst others regarded this as a disadvantage; for instance, less stress is mentioned as an advantage and more stress as a disadvantage of using eHealth.

User-friendliness $(n=64)$, promoting self-care $(n=61)$ and promoting health-behaviour $(n=53)$ were the three largest categories of advantages (A). For disadvantages (D), the largest categories were: equity $(n=48)$, promoting self-care e.g. using a seizure diary and scoring seizure-provoking factors $(n=38)$ and quality criteria, e.g. privacy, user-friendliness $(n=34)$. An overview of all categories is shown in Figure 3.1. 


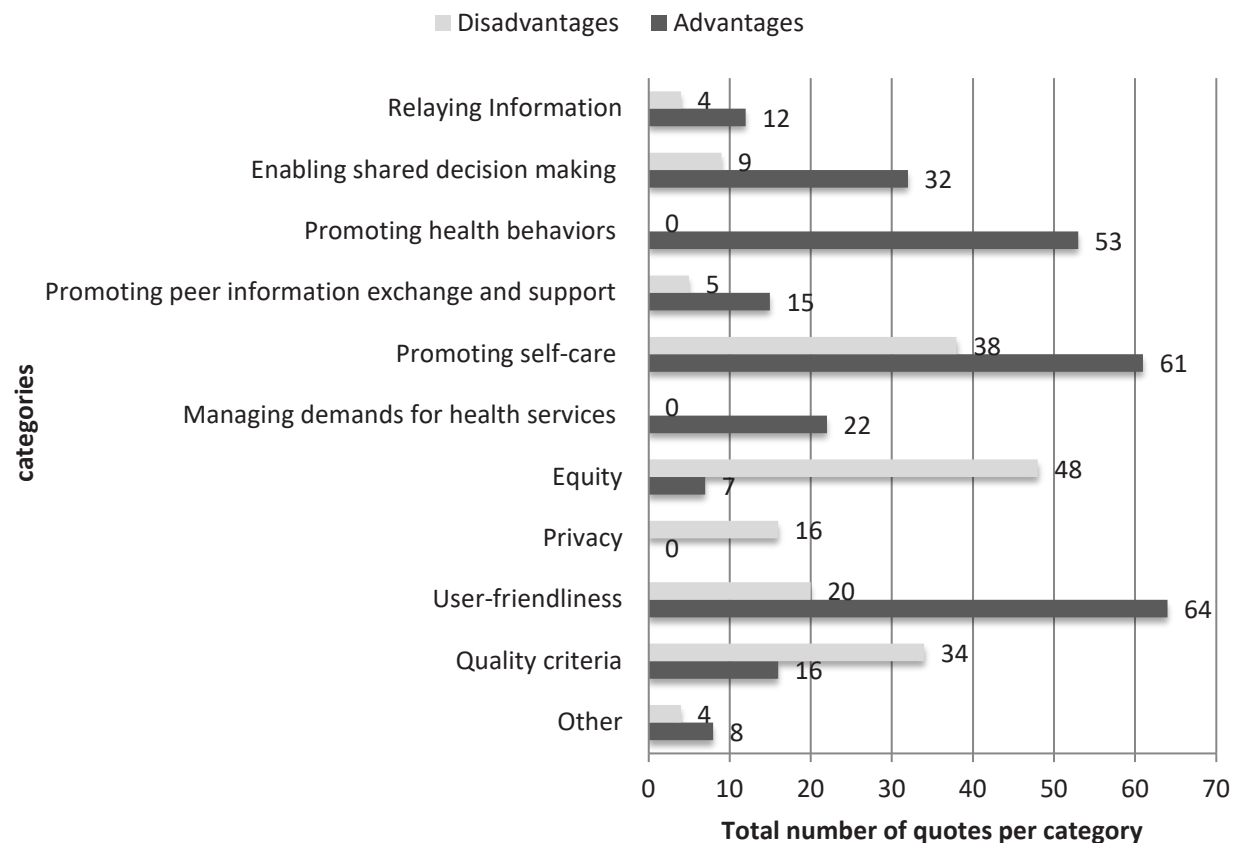

Figure 3.1: Perceived (Dis)-advantages

To give an impression of the quotes in these most-mentioned categories, examples are enumerated per category:

Quotes in the category user-friendliness were 'easy to use' (A), 'always available in contrast to the paper diary' (A), 'very accessible' (A), 'it needs an extra activity' (D) and 'to register ...takes more time than writing it down' (D).

In the category promoting self-care quotes such as 'understanding my seizures and factors influencing having these seizures' (A), 'less stress' (A), 'more independent' (A), 'more stress' (D) and 'medication alarms don't really help, they only confront you with your disease' (D) were used. Quotes as reminders- '...to take medicines for epilepsy' (A) or to 'ask for a refill in time' (A) were labelled in the category promoting health behaviour.

Quotes in the category Equity were, for example, 'you have to go along' (A), 'can everyone work with digital tools?' (D) and 'not fit for elderly people...' (D).

Quality criteria were quoted as 'all kinds of input (seizure, medication, adverse effect) can be combined' (A), 'better presentation of the registered seizures' (A), 'if the battery is low, you can't...' (D) and 'when my phone is lost...' (D).

In addition, the category Privacy consisted of quotes such as 'you are putting personal information on a phone!' (D) and 'is eHealth sufficiently secure?' (D) 
Other categories were less frequently mentioned, an example being the category Demands for health services ( $n=22)$ : quotes were 'quicker diagnoses' (A) 'no more cables needed for an EEG' (A).

In the category Enabling shared decision-making, quotes included 'Allowing me to share information about seizure frequencies with my neurologist even by e-mail' (A), 'digital exchange of data makes treatment easier' (A), 'it can't replace face-toface contact' (D) and 'less contact with neurologist' (D).

The category Promoting peer information exchange and support uses quotes such as: 'information technology could set off an alarm so bystanders know what is happening or what is starting to happen (a seizure)' (A), '...contact always possible by phone' (A). A parent of a child with a mental disability wrote 'you know they have the correct information available when $\mathrm{x}$ is going to sleep somewhere else at the weekend' and 'in a health institution with many healthcare employees, everyone gets the same information in an easy way' $(A)$,

\section{DISCUSSION}

The aim of this study was to explore whether PWE had access to eHealth-tools and whether they perceived eHealth as a useable aid in their self-care. This study focused on three topics; each of these will be discussed separately.

Finally, Internet penetration in Netherlands in the study period (2013) was 94\% (www.internetlivestats.com/internet-users/netherlands/), and hence unlikely to have affected the accuracy of the results.

\section{Use and content of self-monitoring tools}

A majority of the PWE self-monitor their condition by keeping track of their seizures (62\%) or using a medication reminder (53\%). In addition, 48 percent of the respondents monitor on average two to three other events. Most monitoring is not, however, performed with eHealth-tools. People with other chronic conditions monitor their condition less frequently (40\%) and the percentage of eHealth-tool users in this group is smaller (10\% use a computer and $3 \%$ use a smartphone $)^{8}$ than in the group of PWE.

Reasons for the differences between the two groups could be (1) that the respondents in our study were younger (mean age 38.3 y) than those with other chronic conditions (mean age $>60 y$ ), or (2) that epilepsy is a chronic condition often with onset early in life, and so PWE learn earlier to keep track of events influencing their condition (e.g. selfmonitoring).

In spite of evidence that eHealth-tools such as e-medication feedback ${ }^{9}$ and e-diaries ${ }^{10}$ improve adherence, our study shows that most respondents did not use these eHealth- 
tools and, therefore, could not benefit from them. A reason for not using eHealth-tools might be that the above-mentioned tools "seizure tracker" and "My seizure diary" are not available in Dutch.

\section{Possession of hardware.}

We compared our data with data gathered in the same year (2013) of the general population.

It seems that a lower percentage of PWE own a computer or smartphone than the general population (i.e. computer $82 \%$ vs $95 \%$ and smartphone $37 \%$ vs $72 \%$ ). ${ }^{23}$ However, no differences were found between males and females in this regard, in line with findings in the Dutch population. ${ }^{23}$

In our study population, the use of a computer in the four age groups and the use of a smartphone or tablet showed trends similar to the general Dutch population. E.g. younger people are more likely to own a computer, smartphone or tablet, than older people. $^{23}$

However, it has been shown that in the general population, it is mainly the oldest age group who do not own a computer or smartphone ${ }^{23}$, whereas in the present study, the lower percentage is observed in a younger age-group: $45-64$ years.

\section{Advantages and/or disadvantages of using eHealth-tools}

Promoting self-care was the most mentioned disadvantage and the second most mentioned advantage. Those who called this an advantage said in most cases that it could give them insight into their seizure frequency and related events in a way that prepared them for the consultation with the neurologist. This supports the idea that eHealth-tools can be an advantage in the process of shared decision-making ${ }^{3,13}$; this was also one of the largest categories in our study. In contrast, those who called promoting self-care a disadvantage focused more on emotions, such as confrontation with their disease, emotional stress or defining them as patients. The fact that equity was seen as an important disadvantage suggests that people are concerned that not everyone is able to work with eHealth-tools.

EHealth-tools can be helpful for PWE in self-management, including monitoring and managing symptoms (self-care and adherence to treatment and lifestyle regimens (health behaviour)). ${ }^{3-5}$ On the one hand, respondents seem to agree with this statement by referring to the promotion of self-care and health behaviour as an advantage of eHealth. ${ }^{15-17}$ On the other hand, the disadvantages mentioned (equity, perceived lack of privacy) seem to indicate that PWE perceive problems in their ability to use eHealth-tools, and also that they perceive a negative effect on their psychosocial wellbeing. It is, however, possible that PWE have no clear picture of what eHealth is or what eHealth-tools can do for them. This phenomenon was also observed in the annual eHealth-monitor report by the Dutch government. Respondents in this research study gave contradictory 
answers: the same respondent answered 'yes' when asked if they accessed their Personal Medical Record digitally and 'no' to having access to the internet. ${ }^{8}$

\section{Limitations of this study}

This study is prone to several limitations. Firstly, the population is relatively heterogeneous, being comprised of respondents with different types of epilepsy. Hence, certain factors, such as severity of disease or cognitive deficits, may have influenced the use of and intention to use eHealth. Secondly, although we achieved a respectable response rate of $57.1 \%$, it is possible that there is a selection-bias within our sample, as reasons for not responding are not known. Thirdly, the questionnaire was relatively short and lacked some possible important factors, as income and educational level, which have been proven relevant in other studies. ${ }^{18,24}$ Fourthly, as this was a cross-sectional design, we are unable to view trends over time.

\section{CONCLUSION}

In this study, the proportion of PWE who own a computer or smartphone is lower than in the Dutch population. PWE monitor their condition and several events influencing it, but they tend to use pen and paper most frequently, rather than eHealth-tools. Issues which PWE perceive as being (dis)advantages of using eHealth or eHealth-tools are diverse and sometimes contradictory.

The advantages mentioned - supporting PWE in their self-care or enabling them to make shared decisions - are both important factors in stimulating self-management. Selfmanagement is a key component in focusing on quality of life and care for those with chronic conditions such as epilepsy. It is, therefore, important to focus on methods to implement eHealth tools for these patients.

In light of the disadvantages mentioned, we suggest a patient-tailored approach in order to solve the perceived problems. This could improve the implementation of eHealth-tools for self-monitoring by PWE.

Knowing more about the perception of eHealth by PWE makes it easier to develop strategies to support them in using eHealth-tools in their self-care. Further structured research is, therefore, necessary and should involve PWE in developing these eHealthtools. 


\section{REFERENCES}

1. Epilepsy Fact sheet N*999. In: World Health Organisation; 2015.

2. Raad voor de Volksgezondheid (RVZ). Gezondheid 2.0. In; 2010.

3. Ouwens M, van der Burg S, Faber M, van der Weijden T. Shared Decision Making \& Zelfmanagement. In: Scientific Institute for Quality of Healthcare; 2012.

4. Groenewegen A, Tofighy A, Ryvlin P, Steinhoff BJ, Dedeken P. Measures for improving treatment outcomes for patients with epilepsy: results from a large multinational patient-physician survey. Epilepsy Behav 2014; 34: 58-67.

5. Barlow J, Wright C, Sheasby J, Turner A, Hainsworth J. Self-management approaches for people with chronic conditions: a review. Patient Education and Counseling 2002; 48: 177-187.

6. Krijgsman J, Klein Woltering G. Whitepaper Ordening in de wereld van eHealth. In: Nictiz; 2012.

7. Murray E, Burns J, See TS, Lai R, Nazareth I. Interactive Health Communication Applications for people with chronic disease. Cochrane Database Syst Rev 2005: CD004274.

8. Krijgsman J, Peeters J, Burghouts A, de Jong J, Heijmans M, Beenkens F, et al. 'Dan kan ik er misschien zelf iets aan doen': tussenrapportage eHealth-monitor 2015.

9. Demonceau J, Ruppar T, Kristanto P, Hughes DA, Fargher E, Kardas P, et al. Identification and Assessment of Adherence-Enhancing Interventions in Studies Assessing Medication Adherence Through Electronically Compiled Drug Dosing Histories: A Systematic Literature Review and Meta-Analysis. Drugs 2013;73: 545562.

10. Fisher RS, Blum DE, DiVentura B, Vannest J, Hixson JD, Moss R, et al. Seizure diaries for clinical research and practice: limitations and future prospects. Epilepsy Behav 2012; 24: 304-10.

11. Eland-de Kok P, van Os-Medendorp H, Vergouwe-Meijer A, Bruijnzeel-Koomen C, Ros W. A systematic review of the effects of e-health on chronically ill patients. J Clin Nurs 2011; 20: 2997-3010.

12. Notenboom A, Blankers I, Goudriaan R, Groot W. E-health en zelfmanagement: een panacee voor arbeidstekorten en kostenoverschrijdingen in de zorg? In: American Chamber of Commerce (opdrachtgever); 2012.

13. Raad voor de Volksgezondheid (RVZ). De participerende patiënt In: Raad voor de Volgsgezondheid en Zorg; 2013.

14. Shegog R, Bamps YA, Patel A, Kakacek J, Escoffery C, Johnson EK, et al. Managing Epilepsy Well: Emerging e-Tools for epilepsy self-management. Epilepsy Behav 2013; 29: 133-40.

15. Timmer S. eHealth-interventies. In: eHealth in de praktijk: Bohn Stafleu van Loghum; 2011, p. 26-73.

16. Ursum J, Rijken M, Heijmans M, Cardol M, Schellevis F. Zorg voor mensen met een chronische ziekte: organisatie van zorg, zelfmanagement, zelfredzaamheid en participatie. Overzichtstudies. In. Utrecht: NIVEL; 2011. p. 116.

17. Dilorio C, Bamps Y, Walker ER, Escoffery C. Results of a research study evaluating WebEase, an online epilepsy self-management program. Epilepsy Behav 2011; 22: 469-74.

18. Newman L, Biedrzycki K, Baum F. Digital technology use among disadvantaged Australians: implications for equitable consumer participation in digitally-mediated communication and information exchange with health services. Aust Health Rev 2012; 36: 125-9.

19. Pramuka M, Hendrickson R, Van Cott AC. Survey results of Internet and computer usage in veterans with epilepsy. Epilepsy Behav 2010; 17: 366-8.

20. Norman C. eHealth literacy 2.0: problems and opportunities with an evolving concept. J Med Internet Res 2011; 13: e125.

21. van Deursen AJ, van Dijk JA. Internet skills performance tests: are people ready for eHealth? J Med Internet Res 2011; 13: e35.

22. Us Centers For Disease C, Prevention Epilepsy P. Internet use and looking up information online in adults with epilepsy varies by epilepsy status--2013 National Health Interview Survey. Epilepsy Behav 2016; 54: 47-9. 
23. Centraal Bureau voor de Statistiek In: http://statline.cbs.nl/StatWeb/publication/?VW=T \&DM=SLnI\&PA=71098ned\&la=nl. ICT gebruik van personen naar persoonskenmerken 2005-2013.

24. Berner J, Rennemark M, Jogreus C, Anderberg P, Skoldunger A, Wahlberg M, et al. Factors influencing Internet usage in older adults (65 years and above) living in rural and urban Sweden. Health Informatics J 2015; 21: 237-49. 


\section{APPENDIX 3.1}

\section{Questionnaire}

By filling in this survey you contribute in gaining insight into the use of IT technology and apps in patients with epilepsy.

1. General data

a. Gender:

b. Date of birth:

c. I am: person with epilepsy or partner; or parent; or carer of person with epilepsy

2. Do you own a personal computer or laptop? $\mathrm{Y} / \mathrm{N}$

3. Do you own a smartphone? $\mathrm{Y} / \mathrm{N}$

4. Do you own a tablet computer (Android or iPad)? Y/N

5. Do you have any medical apps on your tablet or smartphone? $\mathrm{Y} / \mathrm{N}$

Which one ...

Choose one answer for questions 6-7

6. Do you use a specific aid to help you remember to take your medication?

Which aid do you use?

i Medication box; ii Alarm on your phone; iii Special medication packaging; iv A family member or carer reminds me; v Other....

7. Do you register your seizures?

a. Which tool do you use?

i Paper calendar or diary; ii (personal) computer; iii An app on my tablet or smartphone; iv Other....

b. When did you start registering with this tool?

i Last year; ii Between 1 and 5 years ago iii Over 5 years ago

c. Who usually registers your seizures?

i Myself; ii My partner; iii A family member or caretaker; iv Other....

d. When are the seizures usually registered?

i Directly after; ii Daily; iii Weekly; iv Monthly; v Other...

Multiple answers possible

8. Do you register any other situations or physical factors? Which one

i Frequency of emergency medication usage; ii Intake of medication for emergency reasons iii Forgotten AED intake; iv Change in medication; v Side-effects of medications; vi Diurnal rhythm; vii Stress factors; ix Menstrual cycle; x Intake of contraceptives; xi Other

Choose one answer

9. What is the main reason for registering these situations or factors?

i My neurologist asked me to; ii To find factors influencing my seizures; iii To gain insight into a seizure pattern; iv Because I already have a tool; v Other.... 
Answer on a 4-point Likert scale (1-4): very useful - useful - not useful - not useful at all 10. Is it useful for you to...

i Register seizures; ii Have an intake schedule for medication; iii Use an alarm; iv Have a reminder to take my medication; v Register side-effects; vi Register other situations and factors

Answer on a 4-point Likert scale (1-4) very easy- easy - not easy- not easy at all

11. Is it easy for you to...

i Register seizures; ii Use an intake schedule for medication; iii Use an alarm; iv Remember to take medication; $v$ Register side-effects; vi Register other events and factors

Answer on a 4-point Likert scale (1-4): very important- important - not important- not important at all

12. How important are the following statements for registering seizures?

i It helps me gain insight into my seizure pattern; ii It helps me prepare for the appointment with my healthcare provider; iii It helps me become more involved; iv It helps me gain insight into factors that might influence my seizures; v It helps me make conscious decisions; vi It helps me have more influence on my seizures; vii It helps me have more influence on my treatment plan

13. Can you name two advantages to developing a digital tool or app for epilepsy patients (to register their seizures)?

14. Can you name two disadvantages to developing a digital tool or app for epilepsy patients (to register their seizures)?

Thank you for your help 



\section{CHAPTER}

\section{4}

Process Evaluation of a Multi-Component Self-Management Intervention for Adults

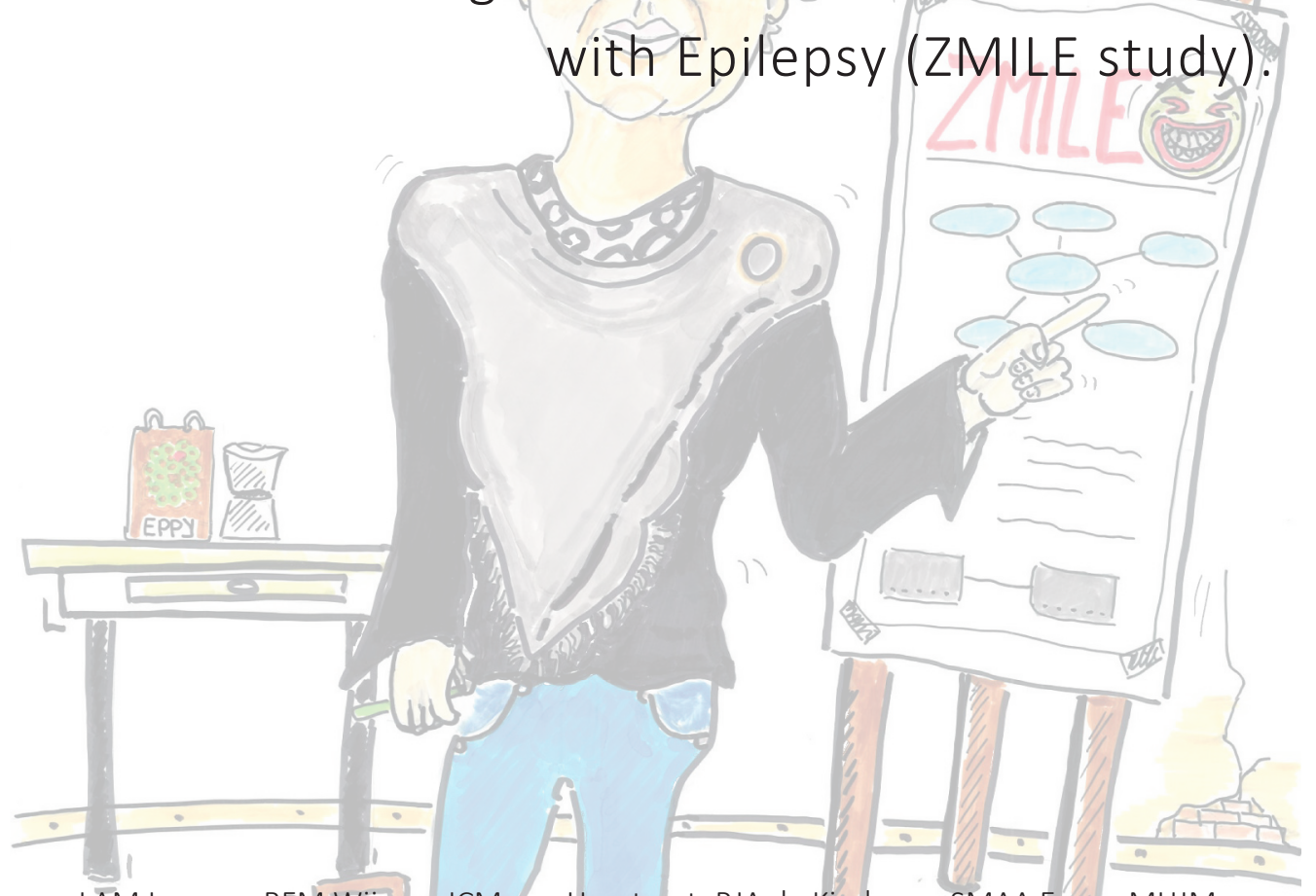

LAM Leenen, BFM Wijnen, JCM van Haastregt, RJA de Kinderen, SMAA Evers, MHJM Majoie. CM van Heugten

Epilepsy \& Behavior 2017; 73: 64-70 


\section{ABSTRACT}

\section{Background}

People with epilepsy need to monitor and manage their symptoms. They, as well as their relatives, have to deal with the psychological burden, reflected in a reduced quality of life. Support in self-management can be of importance. We have developed a multi-component self-management intervention for patients and their relatives $(\mathrm{MCl})$. This eightweek group intervention is conducted by nurse practitioners and consists of six two-hour sessions. The main components are: 1) providing self-management education, 2) stimulating proactive coping and goal-setting and 3) facilitating peer and social support.

This study is a process evaluation to establish the feasibility, fidelity and acceptability of the intervention by assessing performance according to protocol, attendance and adherence, and the opinion of patients, relatives and facilitators about the intervention.

\section{Method}

Study population consists of 52 patients with epilepsy living in the community (e.g. at home), 37 relatives and six facilitators. In this prospective mixed methods study, data were gathered using questionnaires for patients and relatives, registration forms for facilitators and by carrying out semi-structured group interviews with patients, relatives and facilitators.

\section{Results}

Patients and relatives attended a mean of 5.2 sessions. Forty-seven (90\%) patients and $32(86.5 \%)$ relatives attended at least five sessions. The mean group size was 8.1 (SD= 1.3; range 6-10). All elements of the intervention were offered to participants, except for one e-Health tool which was only available at the start of the study. Overall, the sessions were considered useful by patients, their relatives and facilitators. The participation of a relative (social support) and sharing ideas and feelings about having epilepsy with peers (peer support) were rated as important aspects.

\section{Conclusion}

This process evaluation revealed that the $\mathrm{MCl}$ was largely performed according to protocol, attendance rate was high, and participants and facilitators had, on the whole, a favourable opinion about the $\mathrm{MCl}$, and would recommend it to others with epilepsy and their relatives. Overall, the adherence of patients and relatives was high. The $\mathrm{MCl}$ is considered feasible according to patients, relatives and facilitators. Implementation is recommended if the intervention proves to be effective. 


\section{INTRODUCTION}

Epilepsy has considerable effects on the everyday lives of people with epilepsy and their relatives. The seizures, which are the result of sudden electrical discharges in groups of brain cells, manifest clinically in a variety of ways, depending on the area in the brain where the discharges occur. ${ }^{1}$ The psychological burden of having epilepsy is substantial, and is reflected in a reduced quality of life. ${ }^{2,3}$ Furthermore, the societal costs are considerable. ${ }^{4,5}$ Unemployment rates, for instance, are twice as high among people with epilepsy compared to the general population. 6,7 One way to control health care costs is to stimulate self-management and the use of eHealth. ${ }^{8,9}$

People with a chronic condition, such as epilepsy, need to manage their symptoms, by acquiring disease-specific knowledge, adhering to treatment and lifestyle regimens, and coping with the psychosocial consequences of having a chronic condition. ${ }^{10-12}$

In order to change health behaviour, knowledge and skills are required but one should also focus on self-management education Here the emphasis is placed on how patients and relatives can be actively involved in the treatment plan and how skills can be integrated into their everyday lives, e.g. by performing self-monitoring. According to Faber ${ }^{13}$, self-management education should be accompanied by peer support, goal-setting skills, involvement of patients in their treatment plan and self-monitoring. Furthermore, there should be collaborative decision-making, leading to a consensus about the treatment plan (i.e. concordance). ${ }^{14}$ These factors are associated with self-efficacy, defined as the confidence a person has to perform tasks to attain a desired goal. ${ }^{13,15}$

Bradley and colleagues ${ }^{16}$ performed a systematic review to assess the effectiveness of self-management interventions for adults with epilepsy. The main focus of the included studies was either to improve knowledge about epilepsy or to provide strategies to improve compliance. As explained above, however, this is not sufficient to change behaviour.

We have developed a multi-component self-management intervention ( $\mathrm{MCl}$ ) based on the idea that, in order to manage symptoms and physical and psychosocial consequences of epilepsy, patients must also be taught about self-monitoring (by means of eHealth tools) and risk management. Also, in order to alter behaviour, there should be an emphasis on proactive coping, shared decision-making, resulting in concordance, and goal-setting. ${ }^{17}$ The $\mathrm{MCl}$ is based on a self-management intervention specifically designed for stroke patients. ${ }^{18}$ Both interventions had the same structure of group sessions followed by a booster session. Participants were patients with a relative which were both asked to formulate a goal using the framework of a 'proactive action plan' in both interventions. In both interventions theme related information was given and discussed with participants. The themes (topics) were adjusted from stroke to epilepsy in agreement with, among others, representatives of the Epilepsy Association of the Netherlands (EVN, Epilepsie Vereniging Nederland) to make them suitable for people with epilepsy. 
There are some minor differences between both interventions; number of group sessions is one less in the $\mathrm{MCl}$. In the $\mathrm{MCl}$ we focussed more on how to use the frame work in formulating goals instead of formulating a goal on every theme, as was done in the stroke intervention.

The clinical and cost-effectiveness of the $\mathrm{MCl}$ is currently being evaluated in a randomized controlled trial (RCT). ${ }^{19}$ At the same time, a detailed process evaluation has been performed to assess the feasibility of the $\mathrm{MCl}$ and with the aim of analysing the extent to which it was performed according to protocol, the attendance and adherence of patients and their relatives, and the opinion of patients, their relatives and care professionals (i.e. facilitators) about the $\mathrm{MCl}$. The results of this study can be used to optimise the intervention, to facilitate a correct interpretation of the results of the effect evaluation, and eventually facilitate implementation in the regular care of patients with epilepsy.

\section{METHODS}

The $\mathrm{MCl}$ was conducted by six facilitators between May 2014 and January 2016, at three locations in the southern part of The Netherlands (Heeze, Nijmegen and Maastricht). Its observational prospective design combines qualitative and quantitative research methods (mixed methods). The process evaluation is conducted according to the framework of Saunders et $\mathrm{al}^{20}$, addressing performance as described in the protocol (dose delivered), attendance and adherence (reach, dose received exposure and recruitment), and the patients', relatives' and care professionals' opinion about the $\mathrm{MCl}$ (dose received satisfaction).

\section{Multi Component Intervention}

The intervention focuses on increasing participants' understanding of the medical regimen, thus enhancing skills and providing tools to strengthen self-management and communication between patient, relative and health care professionals, and eventually increasing concordance. The $\mathrm{MCl}$ consists of five weekly group sessions of two hours each, followed by a two-hour booster session after three weeks.

The $\mathrm{MCl}$ groups are composed of three to six patients and a number of relatives. Each patient can invite one of his/her relatives to participate in the groups. Since not every patient brings a relative, the intended group size varies between 4 and 12 participants. Structure and content of the $\mathrm{MCl}$ are shown in tables 4.1 and 4.2. The sessions consist of two components: education and practicing goal-setting skills. The educational component includes the topics: self-monitoring; risk evaluation; and shared decision-making/concordance. The aim of this educational part of the sessions was to enhance selfefficacy by discussing and sharing strategies about the topics mentioned above. Two eHealth tools were used for self-monitoring: The Medication Event Monitoring System 
(MEMS; Aardex Ltd., Switzerland) and the smartphone application 'Eppy' (Epilepsy Foundation, The Netherlands). The MEMS are electronic caps which fit on standard pill bottles and register date and time every time the bottle is opened. The data from the electronic caps can be downloaded and used by the care professional to provide feedback to the patient, in order to improve concordance

Table 4.1: Characteristics of the $\mathrm{MCl}$

\begin{tabular}{|c|c|c|}
\hline Participants & \multicolumn{2}{|c|}{ People with epilepsy and their relatives } \\
\hline \multirow[t]{2}{*}{ Facilitators } & \multicolumn{2}{|c|}{ Nurse practitioners, experienced in working with groups of epilepsy patients. } \\
\hline & \multicolumn{2}{|c|}{ Two facilitators per group } \\
\hline \multirow[t]{3}{*}{ Intervention } & Duration & $\begin{array}{l}\text { Eight weeks, with five weekly sessions of two-hours and a two-hour } \\
\text { booster session in week } 8 .\end{array}$ \\
\hline & Group size & $\begin{array}{l}\text { 4- } 6 \text { epilepsy patients with the possibility to bring a relative. Total: } 4-12 \\
\text { participants }\end{array}$ \\
\hline & Setting & Three locations in the Netherlands (Heeze, Maastricht and Nijmegen) \\
\hline \multirow[t]{3}{*}{ Materials } & \multicolumn{2}{|c|}{ A protocol and PowerPoint presentation for the facilitators } \\
\hline & \multicolumn{2}{|c|}{ eHealth-tools for participants: MEMS and Eppy } \\
\hline & \multicolumn{2}{|c|}{ A workbook for the participants } \\
\hline
\end{tabular}

In this process evaluation, MEMS was evaluated as a self-monitor tool that gave patients insight in their pattern of medication intake, by using the electronic gathered data as feedback for patients. Thus, patients were asked to fill the MEMS with either one of their anti-epileptic drugs, or to put the MEMS next to their pill-minder and open the MEMS during their normal routine. Feedback was given by the facilitators during the intervention (session 4-6) and by clinicians every 3 months until the end of the follow-up period. Furthermore, the LCD screen on top of the cap provides the patient with information whether medication was taken or not.

The Eppy is an application, which can be downloaded to smartphone or tablet. It enables patients to keep a seizure diary, to set reminders for taking medication and an alarm mode in case of a seizure. Data gathered in this way can be synchronized to a website owned by the patient.

The goal-setting component is based on the five stages of proactive coping defined by Aspinwall \& Taylor $^{17}:$ 1) resource accumulation, 2) recognition of potential stressors, 3) initial appraisal, 4) preliminary coping efforts, and 5) elicitation and use of feedback concerning initial efforts. Participants formulate proactive action plans structured according to these five stages. ${ }^{21}$ 
Table 4.2: Content of the $\mathrm{MCl}$-sessions

Session Topics

1 Introduction of the $\mathrm{MCl}$, participants and facilitators

Sharing what it means to have epilepsy or having a relative with epilepsy

Sharing expectations about the $\mathrm{MCl}$

2 Introduction of the framework of the 'proactive action plan', which is a 5-step approach for goalsetting.

Participants answering questions which represent the first two steps of the framework

"What do I want to work on?"

"What do I want to achieve?"

Sharing believes, strategies used, emotions and experiences about the topics.

Topic: Self-monitoring

Introduction to self-monitoring. Discussing whether participants are using self-monitoring tools and what kind of tools are used?

Introduction of eHealth-tools (MEMS and 'Eppy').

Participants focus on the third step of the framework on:

"What are the barriers, which prevent me from achieving a goal?" and

"What are possible solutions for the earlier established barriers?"

Sharing believes, strategies used, emotions and experiences about the topics.

4 Topic: Risk- evaluation

What are the perceived risks of having epilepsy, according to the participants? How to deal with perceived risks in daily life?

Participants focus on the fourth step of the framework on:

"What am I actually going to do?"

Sharing believes, strategies used, emotions and experiences about the topics.

5 Topic: Communication with a health care provider

The need to communicate with a health care provider in order to become involved in one's treatment plan.

Participants focus on the last step in the framework which evaluates the previous steps:

"How did it go?"

Sharing believes, strategies used, emotions and experiences about the topics.

Booster Recapitulating the content of the intervention

Evaluating and sharing experiences on participants' action plans

Sharing believes, emotions and experiences about the $\mathrm{MCl}$.

After a two-hour training by the author (LL) to explain the aim and method, the $\mathrm{MCl}$ is conducted by two facilitators (nurse practitioners). They had also followed a two-day course on motivational interviewing techniques, given by a licensed trainer, in which they were taught to ask the participants questions rather than providing a solution to a problem in order to increase self-management skills. Motivational interviewing is a technique to empower participants to set their own sustainable goals and analyse conflicting beliefs. ${ }^{22,} 23$ To support the facilitators, throughout the intervention period, there was a weekly interaction with the researcher (LL). 


\section{Participants}

\section{Patients}

The process evaluation was conducted among the 52 patients who were allocated to the intervention group of the ZMILE-study (RCT) ${ }^{19}$. The patients, included between March 2014 and December 2015, were recruited in the Academic Centre for Epileptology Kempenhaeghe, The Netherlands, by press releases in national epilepsy magazines (Epilepsie, Transmissie) and via social media (Facebook). Inclusion criteria were: aged 18 years or over, living at home, diagnosed with epilepsy and using anti-epileptic drugs, being able to speak and understand Dutch, willing and able to use eHealth devices like a smartphone, and providing written informed consent. Excluded were patients who, based on clinical judgement, were not able or willing to function in group activities and patients who were not able to comprehend topics discussed in the $\mathrm{MCl}$.

\section{Relatives}

Thirty-seven of the 52 patients in the intervention group had a relative who agreed to participate in the intervention. Relative was defined as partner, spouse or other family member closely related to the patient. There were no additional in- or exclusion criteria for the group of relatives.

\section{Facilitators}

Five nurse practitioners (NP) and one nurse were the facilitators of the $\mathrm{MCl}$. They all had experience of working in groups and with epilepsy patients.

\section{Data Collection}

Table 4.3 presents an overview of the measurement instruments used for the process evaluation. 
Table 4.3. Measurements Instruments and Outcome Measures for the process evaluation of the $\mathrm{MCl}$

\begin{tabular}{|c|c|c|c|c|}
\hline & $\begin{array}{l}\text { Evaluation } \\
\text { questionnaire } \\
\text { Participants }\end{array}$ & $\begin{array}{l}\text { Registration } \\
\text { forms }\end{array}$ & $\begin{array}{l}\text { Group Interview } \\
\text { Participants }\end{array}$ & $\begin{array}{l}\text { Group Interview } \\
\text { Facilitators }\end{array}$ \\
\hline \multicolumn{5}{|l|}{ Performance according to protocol } \\
\hline $\begin{array}{l}\text { Were all components of the } \mathrm{MCl} \\
\text { delivered to participants? }\end{array}$ & $x$ & $x$ & & \\
\hline Were e-Health tools used? & & $x$ & $x$ & $x$ \\
\hline Was a proactive action plan formulated? & & $x$ & & \\
\hline \multicolumn{5}{|l|}{ Attendance and Adherence } \\
\hline Attendance rate & & $x$ & & \\
\hline $\begin{array}{l}\text { Number of patients that dropped out of } \\
\text { the intervention }\end{array}$ & & $x$ & & \\
\hline $\begin{array}{l}\text { Number, frequency and duration of the } \\
\text { sessions }\end{array}$ & & $x$ & $x$ & $x$ \\
\hline Recruitment & & & $x$ & $x$ \\
\hline \multicolumn{5}{|l|}{ Opinion on the intervention } \\
\hline Opinion Participants & $x$ & & $x$ & \\
\hline Opinion Relatives & $x$ & & $x$ & \\
\hline Opinion facilitators & & & $x$ & $x$ \\
\hline Barriers & $x$ & $x$ & $x$ & $x$ \\
\hline
\end{tabular}

\section{Patients and relatives}

Patients and relatives filled in a short evaluation questionnaire after completion of the intervention. This questionnaire assessed: 1 ) the perceived usefulness of the six meetings of the $\mathrm{MCl}$ and the eHealth tools on a 7-point Likert scale, (1 = not at all useful - 7= very useful); 2) their opinion on seven statements about the purpose and content of the intervention ( $1=$ totally disagree -7 = totally agree); 3 ) an open question in which participants could express their ideas about the $\mathrm{MCl}$. The questionnaire was either given at the end of the booster session or sent by e-mail if a participant was not able to come to this last session.

All patients and their relatives, who attended at least one session, were invited to give their opinion during one of three group interviews, planned at two locations (Heeze and Maastricht). The interviews were conducted by one researcher (BW) and notes made by another (OG), neither involved in the intervention process. The topics discussed were: elements of the $\mathrm{MCl}$ (presence; usefulness); materials and working methods; opinion about facilitators; location, duration, frequency of $\mathrm{MCl}$; eligibility of the $\mathrm{MCl}$ for epilepsy patients and their relatives.

\section{Facilitators}

The facilitators kept a registration form and were invited to join a group interview. The registration form recorded attendance rate and violations of the intervention protocol (e.g. were any items not discussed? Reasons for not discussing these items). Additionally, 
to this registration form the flipcharts from all sessions, were checked by the researcher (LL) to assess if all topics were discussed.

The group interview was a semi-structured interview, performed by one researcher (BW) and notes made by another (OG); neither had been involved in the intervention process. During this interview the facilitators were invited to evaluate the intervention. The topics dealt with were: training prior to the $\mathrm{MCl}$; their role as a facilitator; recruitment of and eligibility of participants; perceived benefit to the participants; location, duration, frequency of $\mathrm{MCl}$, content of the $\mathrm{MCl}$ and material; and implementation tips.

\section{Data Analysis}

All quantitative data gathered from evaluation questionnaires and registration forms were analysed with descriptive statistics using IBM SPSS statistics Version 21 for MacOs. Results from open-ended questions included in the evaluation questionnaire and group interviews were categorized, based on the content of the answers, by the main author (LL).

\section{Ethical consideration}

The study was approved by the Medical Ethics Committee of Maastricht University Medical Centre/Maastricht University. The study is registered in a public trial register (NTR4484). All participants gave informed consent.

\section{RESULTS}

\section{Response}

Of the 52 patients who embarked on the $\mathrm{MCl}$, five patients, accompanied by three relatives, dropped out of the study. An additional two relatives withdrew from the $\mathrm{MCl}$. The reasons for dropping out are addressed in section 3.4 'Attendance and adherence'. The remaining 47 patients (90\%) and 32 relatives (86.5\%) received and returned the evaluation questionnaire. The group interviews were attended by 15 (28.8\%) patients and five $(13.5 \%)$ relatives. In total, 35 of the 52 patients replied to the request to attend a group interview. Seventeen patients replied that they could not attend any of the group interviews; five patients did not show up. The attendees of the group interviews represented eight of the 13 intervention groups. Five of the six facilitators attended the semi-structured group interview. 


\section{Characteristics of the participants}

Twenty-eight of the 52 patients who started in the $\mathrm{MCl}$-group were male (53.8\%), mean age of the patients was $40.5 \pm 13.5$ years (range $19-66$ years). The majority of patients lived with a relative $(n=36,69.2 \%)$ and were in employment or studying $(n=31,59.6 \%)$.

Of the 37 relatives, 16 were male (43.2\%). They were partners $(n=22,59.5 \%)$; parents $(n=13,35.1 \%)$; other family member $(n=1,2.7 \%)$ and friends $(n=1,2.7 \%)$.

The six facilitators were five female nurse practitioners, and one male nurse. All were nurses experienced in the field of epilepsy

\section{Performance according to protocol (dose delivered)}

Thirteen groups were held at three locations in The Netherlands (Heeze, Nijmegen and Maastricht). Twelve groups were led by two facilitators; one group by one facilitator. The planned second facilitator could not be present. Every intervention group received the planned six sessions, which lasted for two hours each. The mean group size for the 13 groups was 8.1 (SD=1.3; range 6-10). The mean group size for patients was 4.7 (SD= .5; range 4-5) and the mean group size for relatives 3.4 (SD=1.2; range 1-5).

A year after attending the two-day motivational interviewing course, four nurse practitioners started the $\mathrm{MCl}$. Four months later, for logistical reasons, two additional facilitators were needed, and so a nurse practitioner and an experienced nurse were added to the group. They had not attended the motivational interviewing course, which was not available at that time. All six facilitators received the two-hour explanation about the protocol and aim before embarking on their first $\mathrm{MCl}$. The two additional facilitators were given extra training about the purpose of motivational interviewing and ways to perform this technique.

All 47 patients who participated in the $\mathrm{MCl}$, received the eHealth tool MEMS to assist them in self-monitoring. The Eppy eHealth tool could only be used in the first four groups because the application was removed from the appstores (i.e. Apple App Store and Google Play store) and was no longer available.

The participants in the group interviews (patients, relatives and facilitators) reported that all elements of the $\mathrm{MCl}$ were delivered according to protocol. One patient, however, stated that the items: risk-evaluation, self-monitoring and communication with health care providers, were only mentioned briefly, and that the focus was on the proactive action plan.

\section{Attendance and adherence}

Patients attended a mean number of 5.3 sessions (range 1 to 6). Five of the 52 patients withdrew from the $\mathrm{MCl}$ after 1 or 2 session(s). Reasons for discontinuing were: personal health problems ( $n=2), \mathrm{MCl}$ was not what they expected $(n=2)$, and unknown $(n=1)$. 
Relatives attended a mean number of 5 sessions (range 1 to 6 ). Five of the 37 relatives who started the intervention stopped, reasons being: the patient stopped $(n=3)$, health problems ( $n=1)$, and unknown $(n=1)$.

Forty of the 47 patients who received the MEMS as an eHealth tool, reported using this after the $\mathrm{MCl}$ throughout the study; four patients stopped using the tool because: patients were used to their own system, and could not cope with another system ( $n=2)$, stopped with medication ( $n=1)$, no longer interested in using the MEMS $(n=1)$ and unknown ( $n=3)$; 15 patients wanted to continue using the MEMS after completion of the study.

Forty-six of the 47 patients and 30 of the 32 relatives who finished the $\mathrm{MCl}$, formulated a proactive action plan at the end of session five. Examples of such plans were: "taking my medication more regularly" or "improving my sleep time, by not playing on the computer till late at night" and "feeling less responsible for my child/partner with epilepsy". Participants described formulating a proactive action plan with the help of the five-step approach as useful but difficult. This was especially the case for the third step, in which future barriers and solutions for these barriers in establishing a goal, were explored. In the group interviews, most of the patients and relatives stated that they were still using elements of the $\mathrm{MCl}$ (e.g. risk evaluation; talking with a health care provider; using systems to remember medication intake; and using the framework for setting new goals).

\section{Opinion about the intervention}

\section{Patients and relatives}

Table 4.4 shows that overall most patients and relatives had a favourable opinion about the $\mathrm{MCl}$.

Table 4.4: Participants' opinion of the $\mathrm{MCl}(n=79)^{1}$

\begin{tabular}{lll}
\hline & Patients & Relatives \\
\hline Agreement on Statements & $\mathrm{n}(\%)$ & $\mathrm{n}(\%)$ \\
Course is well organised & $42(89 \%)$ & $27(84 \%)$ \\
Purpose of the course is clear & $35(74 \%)$ & $23(72 \%)$ \\
The course was easy to understand & $41(87 \%)$ & $27(84 \%)$ \\
I have learned skills which help me in managing epilepsy & $35(74 \%)$ & $25(78 \%)$ \\
The link between the different parts of the course are clear & $38(81 \%)$ & $30(94 \%)$ \\
The eHealth-tools used, are of additional value in managing epilepsy & $31(66 \%)$ & $22(69 \%)^{2}$ \\
I recommend this course to other people with epilepsy & $39(83 \%)$ & $27(84 \%)$ \\
\hline
\end{tabular}

${ }^{1}$ Total number of patients $n=47$ and relatives $n=32$ that completed the $\mathrm{MCl} .{ }^{2}$ Relatives responded often with NA

In the group interviews, participants, i.e. patients and relatives, reported that the number (6) and duration (2 hours) of the sessions was good. Some participants, however, would 
have preferred to have a booster session after six to twelve weeks, instead of after three weeks.

Participants indicated the optimal group size to be 10 to 12 participants. The material (workbook, sheets) was considered to be sufficient, but perhaps the workbook could have been made available beforehand or be web-based.

According to the evaluation questionnaires, a majority of the participants perceived the sessions to be useful (>74\%). The usefulness of eHealth tools was rated lower, especially by the patients (range 43\%-72\%) (for further details see Supplementary Material 4.5). They stated in the group interviews that the MEMS could only be used for one kind of medication and that it was too fragile to be used by patients with seizures. After being dropped during a seizure, one of the patients' MEMS broke. Only a few of the patients of the group interview used 'Eppy', but they stated that it helped them with their selfmonitoring activities; therefore, a similar eHealth tool should be made available.

In the group interviews, half of the participants reported that they had expected the $\mathrm{MCl}$ to be more of an educational programme, e.g. more education about specific topics such as how and when to use rescue medication; types of seizures, etc.

Important aspects of the $\mathrm{MCl}$ reported by most participants during the group interviews or on the evaluation questionnaires were: the participation of a relative (social support) and sharing ideas and feelings about having epilepsy with peers (peer support).

According to patients and relatives, no additional in- or exclusion criteria are needed for patients or relatives if they want to attend the $\mathrm{MCl}$. Also, in their opinion, there is no need to format more homogeneous groups, for instance based on age or educational level.

\section{Facilitators}

In their group interview, the facilitators reported that the number (6) and duration (2 hours) of the sessions was good and the group size should optimally be between 10-12 participants. In order to follow up the action plan of the participants, facilitators preferred to have the booster session later, for instance after 3 months, or another kind of follow-up, such as a consultation with a nurse practitioner. All facilitators stated that being involved in the $\mathrm{MCl}$ required a considerable investment of time, because it was not embedded in their regular timetable. They also argued, however, that continuing with the $\mathrm{MCl}$ is a way of integrating self-management support into everyday practice, since it is part of their core competence framework. ${ }^{24}$ The facilitators who followed the motivational interviewing course indicated that they would have preferred it closer to the start of the intervention. The two-hour training before and the support during the $\mathrm{MCl}$ were valued as useful and satisfactory. Support was especially useful on the subject of eHealth. The facilitators reported that explaining eHealth to the participants was not always easy, because they themselves sometimes experienced a lack of knowledge. In their opinion, the workbook for the participants should be simplified and their own protocol minimized. 


\section{Barriers}

A major barrier was the fact that the eHealth application 'Eppy' could only be used in the first four groups. From group four on (December 2014) participants experienced problems downloading the application or using the app after updating their mobile devices, leading to the decision to discontinue its use. In 2015, the 'Eppy' was removed from the various stores.

Barriers mentioned by participants, which could influence recruitment were: the fact that some locations of the $\mathrm{MCl}$ were not within reach of public transportation, and the lack of information about the role of the relative in the intervention. One of the relatives stated: 'I thought I was only the chauffeur, didn't know I needed to work so hard, but it was well worth it'.

The main barrier mentioned by all facilitators was the fact that they could not followup the participants on their goal-setting tasks, and therefore could not support them further in self-management. They also mentioned that it was often not clear to the relatives that they should play an active role in the intervention.

\section{DISCUSSION}

This process evaluation revealed that the $\mathrm{MCl}$ was largely performed according to protocol.

The overall attendance and adherence rates were high. The participants and the facilitators had a favourable opinion about the $\mathrm{MCl}$ and would recommend the intervention to other people with epilepsy and their relatives. Most patients used the MEMS as an eHealth tool; however, comments were made on the fact that it was not applicable for multiple medications. Some patients also indicated that they stopped, or didn't start using the MEMS, because it caused confusion with their regular method, such as a pillminder. Almost every participant formulated at least one goal in their own proactive action plan.

Although most elements of the $\mathrm{MCl}$ were provided to the participants, there were some deviations from protocol: two facilitators did not receive the motivational interviewing course, one group had only one facilitator, and the 'Eppy', eHealth application, was no longer applicable for downloading for the last nine groups.

It is difficult to compare these results with other self-management interventions for people with epilepsy as process evaluations in this field are lacking. ${ }^{16}$ When compared with the 'Plan Ahead 'intervention, a comparable self-management intervention for stroke patients and their partners ${ }^{25}$, we find similarities and differences. Similarities, in addition to the high attendance rate, include the value patients and relatives placed on peer- and social support, especially in sharing and comparing ideas about how to deal with the consequences of having epilepsy or having a relative with epilepsy. A similar 
suggestion was to plan the booster session at a later time. Also, in both interventions, the facilitators reported the need to emphasize the active role of the relative, and the wish to receive their training closer to the start of the $\mathrm{MCl}$. The main difference with the 'Plan ahead' intervention was the fact that the proactive action plan in the $\mathrm{MCl}$ was executed according to protocol, and participants assessed this part of the $\mathrm{MCl}$ as being difficult, but useful.

The $\mathrm{MCl}$ aims to change behaviour of participants but changing health behaviour is a process which acquires maintenance, since most of the intended behavioural changes tend to diminish after the active intervention phase. ${ }^{26,27}$ How maintenance of health behaviour should be executed and by whom, requires further discussion. ${ }^{28}$ Both participants and facilitators mentioned the need for a follow-up or maintenance plan. This could be realised by, for example, a booster session after 3 months instead of the 3 weeks we used in the $\mathrm{MCl}$, or by a follow-up consultation by a nurse practitioner, who was involved in the $\mathrm{MCl}$ of these participants. ${ }^{28}$

This study also has some limitations. First, the attendance rate for the participant group interviews was rather low, which may have led to bias in the results. Second, performance according to protocol was measured quantitatively (dose delivered) using registration forms administered by the facilitators. However, the quality (fidelity) of the performance of the facilitators was not assessed; this should be done by observation or video-recording, something we considered to be too intrusive for the participants. ${ }^{29}$ Third, it is possible that participants and facilitators gave socially desirable answers. To limit this risk, all interviews were carried out by two independent interviewers, not involved in the $\mathrm{MCl}$, and the evaluation questionnaires, which were administered by the participants, were anonymous for the facilitators.

\section{CONCLUSSION}

In conclusion, according to patients, relatives and facilitators, the $\mathrm{MCl}$ is feasible, although improvement is still possible. Suggested improvements are: planning the booster session at a later time, managing expectations about the $\mathrm{MCl}$. Furthermore, eHealth and eHealth-tools should remain part of the intervention, although discussion and or further research is still needed to establish whether using MEMS is the best way to give patients insight in their medication intake pattern.

Based on this study, we recommend making some minor adjustments to the $\mathrm{MCl}$ and implementing the $\mathrm{MCl}$ in regular care when it proves to be (cost)-effective. 


\section{REFERENCES}

1. Epilepsy Fact sheet N*999. In: World Health Organisation; 2015.

2. Jacoby A, Baker GA. Quality-of-life trajectories in epilepsy: a review of the literature. Epilepsy Behav 2008; 12: 557-71.

3. Taylor RS, Sander JW, Taylor RJ, Baker GA. Predictors of health-related quality of life and costs in adults with epilepsy: a systematic review. Epilepsia 2011; 52: 2168-80.

4. Kotsopoulos IAW, Evers SMAA, Ament AJHA, De Krom MCTFM. Estimating the Costs of Epilepsy: An International Comparison of Epilepsy Cost Studies. Epilepsia 2001; 42: 634-640.

5. Kotsopoulos IA, Evers SM, Ament AJ, Kessels FG, de Krom MC, Twellaar M, et al. The costs of epilepsy in three different populations of patients with epilepsy. Epilepsy Res 2003; 54: 131-40.

6. Elwes RD, Marshall J, Beattie A, Newman PK. Epilepsy and employment. A community based survey in an area of high unemployment. J Neurol Neurosurg Psychiatry 1991; 54: 200-3.

7. Pato Pato A, Cebrián Pérez E, Cimas Hernando I, Lorenzo González JR, Rodríguez Constenla I, Gude Sampedro F. Analysis of direct, indirect, and intangible costs of epilepsy. Neurología (English Edition) 2011; 26: $32-38$

8. Ursum J, Rijken M, Heijmans M, Cardol M, Schellevis F. Zorg voor mensen met een chronische ziekte: organisatie van zorg, zelfmanagement, zelfredzaamheid en participatie. Overzichtstudies. In. Utrecht: NIVEL; 2011; p. 116.

9. Raad voor de Volksgezondheid (RVZ). De participerende patiënt In: Raad voor de Volgsgezondheid en Zorg; 2013.

10. Barlow J, Wright C, Sheasby J, Turner A, Hainsworth J. Self-management approaches for people with chronic conditions: a review. Patient Education and Counseling 2002; 48: 177-187.

11. Bodenheimer T, Lorig K, Holman H, Grumbach K. Patient self-management of chronic disease in primary care. JAMA 2002; 288: 2469-75.

12. Ouwens M, van der Burg S, Faber M, van der Weijden T. Shared Decision Making \& Zelfmanagement. In: Scientific Institute for Quality of Healthcare; 2012

13. Faber M, Harmsen M, van der Burg S, van der Weijden T. Gezamenlijke besluitvorming \& Zelfmanagement: Een literatuuronderzoek naar de effectiviteit ven en naar voorwaarden voor succes. In. Nijmegen: Scientific Institute for Quality of Healthcare (IQ healthcare); 2013.

14. Haynes RB, Ackloo E, Sahota N, McDonald HP, Yao X. Interventions for enhancing medication adherence. Cochrane Database Syst Rev 2008: CD000011.

15. Lemmens KM, Nieboer AP, van Schayck CP, Asin JD, Huijsman R. A model to evaluate quality and effectiveness of disease management. Qual Saf Health Care 2008; 17: 447-53.

16. Bradley PM, Lindsay B, Fleeman N. Care delivery and self management strategies for adults with epilepsy. Cochrane Database Syst Rev 2016; 2: CD006244.

17. Aspinwall LG, Taylor SE. A stitch in time: self-regulation and proactive coping. Psychol Bull 1997; 121: 41736.

18. Tielemans NS, Schepers VP, Visser-Meily JM, van Erp J, Eijkenaar M, van Heugten CM. The Restore4Stroke self-management intervention 'Plan ahead!': rationale and description of the treatment protocol based on proactive action planning. Clin Rehabil 2014; 28: 530-40.

19. Leenen LA, Wijnen BF, de Kinderen RJ, Majoie MH, van Heugten CM, Evers SM. (Cost)-effectiveness of a multi-component intervention for adults with epilepsy: study protocol of a Dutch randomized controlled trial (ZMILE study). BMC Neurol 2014; 14: 255.

20. Saunders RP, Evans MH, Joshi P. Developing a process-evaluation plan for assessing health promotion program implementation: a how-to guide. Health Promot Pract 2005; 6: 134-47.

21. Thoolen B, de Ridder D, Bensing J, Gorter K, Rutten G. Beyond Good Intentions: the development and evaluation of a proactive self-management course for patients recently diagnosed with type 2 diabetes. Health Educ Res 2008; 23: 53-61. 
22. Miller WR, Rollnick S. Ten things that motivational interviewing is not. Behav Cogn Psychother 2009; 37: 129-40.

23. Miller WR, Stephen R. Motivational interviewing : preparing people for change. 3th ed. New York: Guilford Press; 2012.

24. Fact sheet The Nurse Practitioner in the Netherlands. In; http://venvnvs.nl/wp-content/uploads/sites/164/2015/08/2015-10-30-Factsheet-Nurse-Practitioner-Netherlands-2015.pdf

25. Tielemans NS, Schepers VP, Visser-Melly JM, van Haastregt JC, van Veen WJ, van Stralen HE, et al. Process evaluation of the Restore4stroke Self-Management intervention 'Plan Ahead!': a stroke-specific self-management intervention. Clin Rehabil 2015; 30: 1175-1185.

26. Ryan P. Integrated Theory of Health Behavior Change: background and intervention development. Clin Nurse Spec 2009; 23: 161-70; quiz 171-2.

27. Ory MG, Lee Smith M, Mier N, Wernicke MM. The science of sustaining health behavior change: the health maintenance consortium. Am J Health Behav 2010; 34: 647-59.

28. Hughes SL, Seymour RB, Campbell RT, Desai P, Huber G, Chang HJ. Fit and Strong!: Bolstering Maintenance of Physical Activity Among Older Adults With Lower-extremity Osteoarthritis. American journal of health behavior 2010; 34: 750-763.

29. Linnan L, Steckler A. Process evaluation for public health interventions and research:An Overview. In: Process evaluation for public health interventions and research; 2002. 


\section{SUPPLEMENTARY MATERIAL 4.5}

\section{Usefullness of the $\mathrm{MCl}$}

Supplementary Table 4.5: Number of participants that regarded elements of the $\mathrm{MCl}$ useful $(\mathrm{n}=79)^{1}$

\begin{tabular}{|c|c|c|c|c|c|c|c|}
\hline \multirow[b]{2}{*}{$N(\%)$} & \multicolumn{2}{|l|}{ Useful } & \multicolumn{2}{|c|}{$\begin{array}{l}\text { Nor useful /nor not } \\
\text { useful }\end{array}$} & \multicolumn{2}{|c|}{ Not useful } & \multirow[t]{2}{*}{$\begin{array}{l}\text { Overall } \\
\text { Missing }^{2}\end{array}$} \\
\hline & Patients & Relatives & Patients & Relatives & Patient & Relatives & \\
\hline Session 1 & $35(74 \%)$ & $28(88 \%)$ & $7(15 \%)$ & $0(0 \%)$ & $3(6 \%)$ & $1(3 \%)$ & $5(6 \%)$ \\
\hline Session 2 & $39(83 \%)$ & $27(84 \%)$ & $4(9 \%)$ & $1(3 \%)$ & $4(9 \%)$ & $1(3 \%)$ & $3(4 \%)$ \\
\hline Session 3 & $37(78 \%)$ & $25(78 \%)$ & $4(9 \%)$ & $4(13 \%)$ & $5(11 \%)$ & $1(3 \%)$ & $3(4 \%)$ \\
\hline Session 4 & $40(85 \%)$ & $25(78 \%)$ & $4(9 \%)$ & $3(9 \%)$ & $3(6 \%)$ & $1(3 \%)$ & $3(4 \%)$ \\
\hline Session 5 & $36(77 \%)$ & $26(81 \%)$ & $3(6 \%)$ & $0(0 \%)$ & $3(6 \%)$ & $0(0 \%)$ & $11(14 \%)$ \\
\hline Booster session & $37(78 \%)$ & $26(81 \%)$ & $5(11 \%)$ & $2(6 \%)$ & $1(2 \%)$ & $0(0 \%)$ & $8(10 \%)$ \\
\hline eHealth-tools: MEMS & $33(70 \%)$ & $23(72 \%)$ & $7(15 \%)$ & $2(6 \%)$ & $6(13 \%)$ & $3(9 \%)$ & $5(6 \%)$ \\
\hline eHealth-tools: 'Eppy' & $20(43 \%)$ & $18(56 \%)$ & $6(13 \%)$ & $3(9 \%)$ & $12(26 \%$ & $2(6 \%)$ & $18(23 \%)$ \\
\hline
\end{tabular}

${ }^{1}$ Total number of patients $n=47$ and relatives $n=32$ that completed the $\mathrm{MCl}$. ${ }^{2}$ Total of missing is the combined number of missing values and questions answered with NA. Reasons for answering questions with NA were e.g. not attending sessions or using the eHealth-tools. 



\section{CHAPTER}

An Economic Evaluation of a Multicomponent Self-Management Intervention for Adults with

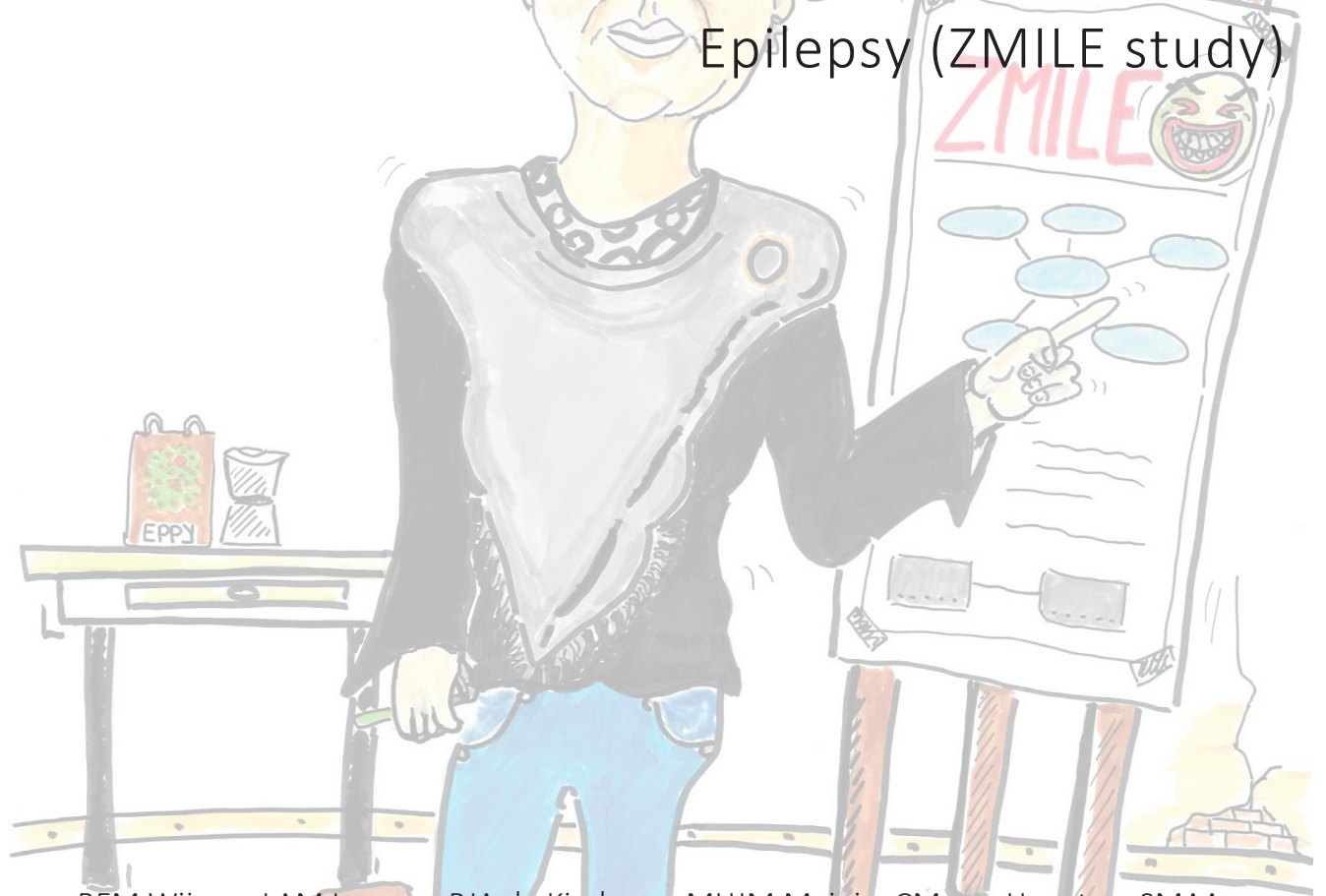

BFM Wijnen, LAM Leenen, RJA de Kinderen, MHJM Majoie, CM van Heugten, SMAA Evers

Epilepsia 2017; 58: 1398-1408

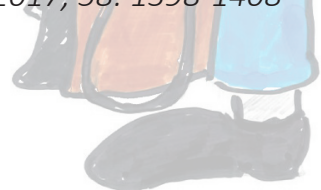




\section{ABSTRACT}

\section{Objective}

The objective of this (trial-based) economic evaluation was, from a societal perspective, to compare the cost-effectiveness of a multi component self-management intervention $(\mathrm{MCl})$ compared to care as usual (CAU) in adult patients with epilepsy with a follow-up over a 12-month period.

\section{Methods}

In a randomized-controlled trial, participants were randomized into intervention or CAU group. Adherence, self-efficacy (Epilepsy Self-Efficacy Scale [ESES]), quality-adjusted life years (QALYS), healthcare costs, production losses, and patient and family costs were assessed at baseline and during the 12-month study period. Incremental cost-effectiveness ratios (ICERs) (i.e., cost per increased adherence, self-efficacy, or QALY), and cost-effectiveness acceptability curves were calculated.

\section{Results}

In total 102 patients were included in the study, of whom 52 in the intervention group. Adherence rates over 6 months were $63.7 \%$ for the CAU group and $75.9 \%$ for the intervention group. Adherence, ESES and quality of life were not significantly different between groups. An ICER of $€ 54$ per point increase in ESES-score at 6 months and $€ 1105$ per point increase at 12 months follow-up was found. The intervention resulted in an ICER of $€ 88$ per percentage of adherence increase at 6 months. ICERs of $€ 8,272$ and $€ 15,144$ per QALY gained were found at 6- and 12-month follow-up, respectively.

\section{Discussion}

Although no statistically significant difference was found after baseline adjustments, costeffectiveness estimates for $\mathrm{MCl}$ appear promising. As rules of inference are arbitrary, it has been argued that decisions should be based only on the net benefits, irrespective of whether differences are statistically significant. Hence, the $\mathrm{MCl}$ may be a cost-effective addition to the current standard care for adults with epilepsy. 


\section{INTRODUCTION}

In Europe, the prevalence of epilepsy is estimated to be 5 per 1,000 inhabitants. ${ }^{1}$ Because life expectancy is rising, resulting in an aging population, the expectation is that the number of people with a chronic condition such as epilepsy will increase. ${ }^{2}$ In terms of economic impact on society, epilepsy is highly cost-intensive with respect to diagnosis, treatment, and hospitalizations due to seizures, as well as unemployment and household work. $^{3,4}$ Furthermore, uncontrolled seizures negatively impact the quality of life (QoL) of patients with epilepsy. ${ }^{1}$ Because the healthcare budget is insufficient to cover all healthcare expenses ${ }^{5}$, economic evaluations are becoming a common requirement for reimbursement decisions. ${ }^{6,7}$ Budget constraints challenge policymakers to make the most cost-effective and health producing decisions when allocating limited resources.

People with epilepsy are in many ways their own primary carers; well-controlled epilepsy often results in less productivity losses, hospitalizations, and other use of (healthcare) resources. ${ }^{8}$ Higher quality of self-care and reduced healthcare costs, provision of information, and adequate problem-solving skills are needed. Self-management education programs are designed to meet this need. ${ }^{9}$ Evidence from previous controlled clinical trials has indicated positive effects of self-management programs on improving clinical outcomes and their potential to reduce costs for chronic disorders. ${ }^{9,10}$ However, the Managing Epilepsy Well (MEW) network has revealed a lack of evidence-based programs for epilepsy self-management education. ${ }^{11}$ One of the mechanisms responsible for the improvements in health status, demonstrated by those attending self-management programs, is self-efficacy. ${ }^{9}$ Furthermore, the effectiveness of an antiepileptic drug (AED) treatment not only depends on the type of AED; the patients' attitude to it is also of great importance. ${ }^{12}$ Hence, adherence, or more precisely, concordance, with medical treatment is closely linked to the patients' ability to self-manage their disease, and is shown to be an important factor in determining QoL. ${ }^{13}$ Concordance reflects a consensual agreement about taking AEDs that has been established between patient and practitioner. ${ }^{14}$

In this study, a full economic evaluation was performed that examined the cost-effectiveness of a multicomponent intervention ( $\mathrm{MCl}$ ) aimed at increasing self-management skills for people with epilepsy. Hence, the aim of this study was to compare, from a societal perspective, the cost-effectiveness (i.e., cost per improved adherence or self-efficacy) and cost-utility (i.e., cost per quality of life years [QALY]gained) of the $\mathrm{MCl}$ with care as usual (CAU) in adult patients with epilepsy over a period of 6 months with an extended follow-up of 12 months for the $\mathrm{MCl}$ group. 


\section{METHODS}

\section{Design}

This trial-based economic evaluation was part of a randomized controlled trial (RCT) in which the clinical effectiveness of the $\mathrm{MCl}$ was assessed. Randomization was performed using sealed envelopes by an independent person. The study consisted of two parallel groups in which participants were randomly allocated to either the $\mathrm{MCl}$ group (a selfmanagement education program with e-Health interventions) or CAU. The control group received CAU. Participants in the intervention group were followed for 12 months, and the CAU group for 6 months. After 6 months, patients from the control group were also given the opportunity of receiving $\mathrm{MCl}$; this did not form part of the study. The protocol of this study has been described previously in Leenen and Wijnen et al. (2014). ${ }^{15}$ An overview of all relevant measurements for this economic evaluation is presented in Figure 5.1.

\section{Participants \& Setting}

Adult patients, aged 18 years and over who were diagnosed with epilepsy, who lived at home, used AEDs, understood the Dutch language, and were willing and able (based on neurologists' opinion) to use e-Health devices belonging to the $\mathrm{MCl}$, were eligible to participate in this study. Patients were excluded if they were not able or willing to function in group activities, or when it was expected, based on clinical judgment, they were not able to comprehend topics discussed within the $\mathrm{MCl}$ (e.g., patients with cognitive deficits). Patients were recruited in the outpatient clinics of Kempenhaeghe and by means of national epilepsy magazines and social media. The inclusion started in March 2014 and the last follow-up measurements were carried out in November 2016.

\section{Multi-component intervention}

The $\mathrm{MCl}$ consists of the following: (1) group sessions, (2) the Medication Event Monitoring System (MEMS; Aardex Ltd., Switzerland); (3) a smartphone application "Eppy" (Epilepsy Foundation, The Netherlands); and (4) an Internet accessible patient database. The group sessions consisted of groups of three to five patients and additionally family members and/or friends. During the first 5 weeks, group sessions took place once a week, followed by a booster session 4 weeks later. The group sessions lasted for $2 \mathrm{~h}$ and were led by two nurse practitioners, with experience in working in epilepsy patient groups. During the course, participants practiced with the five stages of proactive coping described by Aspinwall and Taylor ${ }^{16}$ and three fixed epilepsy- related themes.15 These five stages were (1) resource accumulation, (2) recognition of potential stressors, (3) initial appraisal, (4) preliminary coping efforts, and (5) elicitation and use of feedback concerning initial efforts. The three epilepsy-related themes were (1) self-monitoring and self- 
monitoring (e-Health) tools; (2) risk-management; and (3) shared decision-making/concordance. Patients were instructed to set goals and to proactively cope with possible pitfalls. The course was based on a self-management program for patients with type 2 diabetes, which was shown to significantly improve self-efficacy. ${ }^{17}$

The MEMS caps were electronic caps that fit on standard pill bottles. They registered the date and time every time the pill bottle was opened. In addition, the MEMS of the intervention group included an LCD screen that provided feedback on the number of times the bottle was opened on a particular day. Patients in the intervention group received feedback on medication adherence based on the MEMS reports during the group sessions and after 3, 6, 9, and 12 months of follow-up. Adherence was expressed as the percentage of days the MEMS bottle was opened according to the AED regimen, calculated as $100 \times$ (number of days with number of openings as prescribed/number of monitored days).

The smartphone application "Eppy" was designed to register seizure frequency and other facts for persons with epilepsy. The Internet-accessible patient database consisted of a web-based interface and overview of the information gathered in "Eppy".

\section{Care as usual}

Patients in the CAU group received no specific intervention; care was not intensified. The CAU group did not follow a standardized protocol. The CAU group also received the MEMS cap but without feedback about their behaviour and without an LCD-screen providing feedback regarding the number of times it was opened on a particular day.

\section{Outcomes}

Self-reported questionnaires were administered to all patients at baseline, 3 months, and 6 months. The intervention group also completed the self-reported questionnaires at 9 and 12 months. Furthermore, adherence, used as a proxy for concordance, was monitored using the MEMS. The questionnaires included in the study were Epilepsy Self-Efficacy Scale-33 item (ESES), the Quality of Life in Epilepsy-31Patient Weighted (QOLIE-31$P)$, the EuroQol 5 dimensions 5 levels (EQ-5D-5L), and a questionnaire regarding (healthcare) resource use over the last 3 months.

The ESES was used to assess the efficacy of self-management education program on epilepsy. Self-efficacy was defined as the confidence to carry out a behaviour necessary to reach a desired goal. ${ }^{18-20}$ The items represent three dimensions of self-management: medication management, seizure management, and general management including safety and health. The total possible scores for the ESES range from 0 to 330 . Higher scores correspond to higher levels of confidence in ability to manage epilepsy. ${ }^{21}$ 


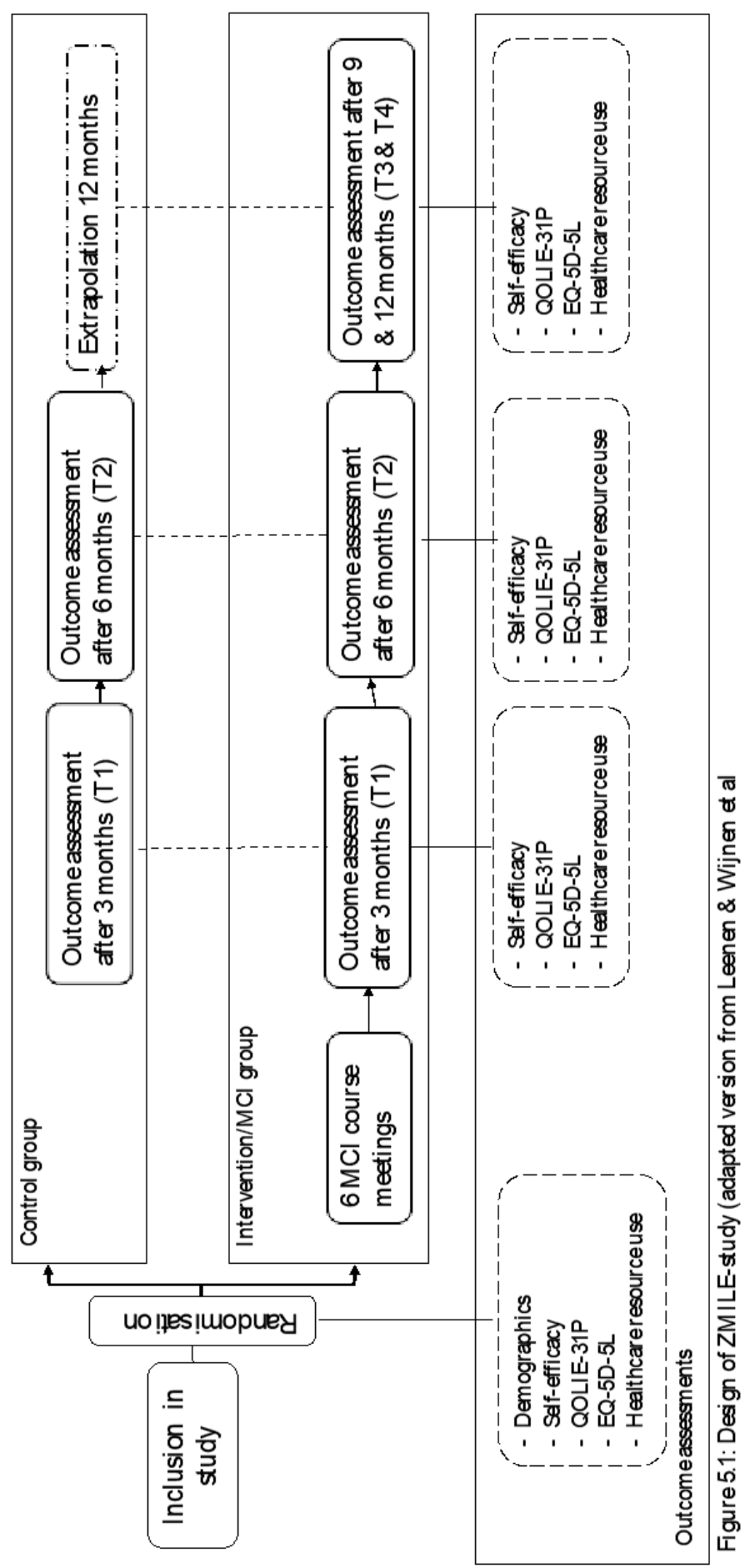


The EQ-5D-5L was used to assess generic QoL expressed in a utility with a value between 0 and 1 , where 1 means perfect health and 0 worst imaginable state of health. ${ }^{22}$ Utilities were derived using the Dutch tariffs. ${ }^{23}$

Disease-specific quality of life was measured with the QOLIE-31-P, which consists of 38 items assessing 7 domains of epilepsy: seizure worry, overall QoL, emotional well-being, energy-fatigue, cognitive functioning, medication effects, social functioning, and an overall score. ${ }^{24}$ The final score ranges from 0 to 100 , in which higher values indicate a better QoL. ${ }^{25}$ Healthcare resource use and patient and family costs were measured using the Medical Cost Questionnaire, which was an adapted version of the Trimbos/iMTA questionnaire for costs associated with psychiatric illness. Productivity costs were measured with the Productivity Cost Questionnaire, both covering 3 months.

A detailed description of the measurement instruments can be found in Leenen and Wijnen et al. ${ }^{15}$

\section{Costs}

This economic evaluation was performed according to the Dutch guidelines for economic evaluations ${ }^{26}$ and the Consolidated Health Economic Evaluation Reporting Standards. ${ }^{27} \mathrm{~A}$ cost-effectiveness (CEA) and cost-utility analysis(CUA) were performed from a societal perspective to estimate the cost per QALY gain.

Costs were divided into four cost categories: intervention costs, healthcare sector costs, costs for patient and family, and productivity costs. The calculation was based on a detailed inventory covering all cost items composed of standardized cost prices from the Dutch manual for costing, and the calculated mean cost prices from the providers. To determine the costs of drugs, the website of the Dutch healthcare institute for the cost of pharmaceuticals (www.medicijnkosten.nl) was used. Intervention costs included the costs of the MEMS and costs associated with the $\mathrm{MCl}$ such as overhead costs, costs for instructors, costs of feedback sessions, and time costs for patients and relatives or friends (if a relative or friend was brought to the group sessions by a patient). Healthcare costs consisted of consultations with healthcare professionals, the use of diagnostic methods, and the frequency of inpatient stay and outpatient treatment. Patient and family costs included the use of formal (paid) care, informal care, and medical devices. The costs for unpaid care were valued using the proxy good method, which values the time spent on informal care at the labour market price of a close market substitute. Productivity costs included productivity losses due to absence from work and were valued using the friction cost method as recommended in the Dutch guidelines. ${ }^{26}$ The friction cost method implies that long-term sick or absent employees will be replaced by employers after a specified friction period based on the average period an employer needs to replace a sick employee. In The Netherlands, a friction period of 85 days is recommended. All costs were indexed for the year 2015. Because the study follow-up was one year, no discounting was performed (for either costs or effects). 


\section{Analyses}

Baseline differences in costs were checked with nonparametric bootstrapping, based on 1,000 bootstrap replications, as the data did not comply with the underlying assumptions of parametric tests using Microsoft Excel 2010. ESES scores, QOLIE-31-P scores, utilities, and demographic variables were assessed for baseline differences using independent ttests (for continuous variables) or Pearson' s chi-square tests (for categorical variables) using IBM SPSS Statistics version 24. Adherence rates were compared using Wilcoxon rank test for repeated measurements. ESES, QOLIE-31-P, and utility follow-up scores were analysed using multi-level analysis with a random intercept for each subject to account for repeated measurements, to correct for potential confounders (i.e., demographics, baseline depression, and anxiety scores), and to account for baseline differences (i.e., by including baseline values as an independent variable). Missing values (i.e., EQ-5D-5L, ESES, QOLIE-31-P, and costs) at baseline were managed using mean imputation ( $n=3$ for CAU group and $n=1$ for intervention group) due to the low number of missing values and as no clinical information was available for these patients.

Missing data at follow-up measurements were dealt with using multiple imputation (5 times). Imputation was based on age, number of medications, employment, seizure frequency at baseline, seizure severity, self-efficacy score, randomization group, health care, patients and family, and productivity costs at each time point (for cost data only), EQ-5D-5L scores at each time point (for EQ-5D data only), ESES scores at each time point (for ESES data only), and QOLIE-31-P score at each time point (for QOLIE-31-P data only). To account for nonnormality of the cost data, predictive mean matching was used in which "real" observed values from similar cases were imputed instead of imputing regression estimates. ${ }^{28,29}$ QALYs were calculated by means of the "under the curve method," in which the time in a certain health state was multiplied by the utility of this health state. ${ }^{30}$ Disease-specific QALYs were calculated based on the QOLIE-31-P by dividing the total QOLIE-31-P score by 100 to obtain disease-specific utility-like scores.

An intention to treat (ITT) analysis was used, meaning that all patients initially included in the study were included in the analyses. For the analysis of the MEMS, this implied that all patients who initiated the MEMS were included in the analysis. Analyses were carried out at 6 months (end of comparative phase) and after 12 months of followup. The CAU group was followed for only 6 months, and the results were extrapolated to 12 months, assuming this period was sufficient to obtain a representative estimation of 12-month follow-up costs. Because adherence was measured using MEMS caps in both groups, which may be seen as an intervention in both groups, the incremental cost-effectiveness ratios (ICERs) for adherence rates was calculated for only the comparative phase (6 months).

ICERs and incremental cost-utility ratios (ICURs) were calculated by dividing the incremental costs by the incremental effects, or QALYS, resulting in the costs per increased adherence, self-efficacy, QALY, or disease-specific QALY of the $\mathrm{MCl}$ as opposed to CAU. 
To quantify the uncertainty around the ICER/ICUR, nonparametric bootstrapping was performed (5,000 times). To show the probability that the $\mathrm{MCl}$ is cost-effective, given different ceiling ratios, a cost-effectiveness acceptability curve (CEAC) was constructed.

In The Netherlands, the Council for Public Health and Health Care proposed an informal ceiling ratio for QALYs between $€ 20,000$ and $€ 80,000$ per QALY, depending on the burden of disease. ${ }^{31}$ Based on this proposition and the disability weight of (severe) epilepsy reported elsewhere ${ }^{32}$, one could assume that the maximum willingness to pay for one QALY is approximately $€ 50,000$.

A regression-based adjustment was performed to account for the difference in baseline costs, (disease-specific) utilities, and ESES scores between both groups. ${ }^{33,34}$

One-way sensitivity analyses were performed by the following: (1) using disease-specific QALYs based on the QOLIE-31-P; (2) applying a healthcare perspective, instead of a societal perspective, as some European countries tend to adopt this; (3) excluding the costs of the MEMS for the CAU group, as patients in the CAU group did not receive any feedback from the MEMS and simply used the device for research purposes; and (4) by using the UK tariff to value the EQ-5D-5L health states.

\section{RESULTS}

\section{Baseline characteristics}

A total of 102 patients were included in the study, 52 of whom were in the intervention group. A large part of the patients reported to have an unpaid job or to be unemployed (61.8\%). There was, however, a significant difference between the intervention group and the CAU group, indicating the intervention group had significantly more patients who had a paid job $(p=0.02)$. Furthermore, the intervention group had a significantly higher score on the ESES $(p=0.02)$. Apart from employment rate and ESES score, no other baseline differences were found (see Table 5.1).

At baseline, healthcare costs and patient and family costs were similar between both groups. Productivity costs were, however, higher in the intervention group, leading to higher costs overall in the intervention group compared to the CAU group (i.e., € 3,338 vs. $€ 2,030)$. Although the two (95\%) confidence intervals of total costs overlap marginally, a baseline correction was made. Unit prices are presented in Supplementary Material 5.1 
Table 5.1: (Baseline) characteristics of the population

\begin{tabular}{|c|c|c|c|}
\hline Characteristics & $\begin{array}{l}\text { Intervention group } \\
(n=52)\end{array}$ & $\begin{array}{l}\text { Control group } \\
(n=51)\end{array}$ & $\begin{array}{l}\text { Total } \\
(n=103)\end{array}$ \\
\hline \multicolumn{4}{|l|}{ Gender } \\
\hline Male & $28(53.9 \%)$ & $23(45.1 \%)$ & $51(49.5 \%)$ \\
\hline Female & $24(46.2 \%)$ & $28(54.9 \%)$ & $52(50.5 \%)$ \\
\hline \multicolumn{4}{|l|}{ Age in years } \\
\hline $18-24$ & $7(13.5 \%)$ & $7(13.7 \%)$ & $14(13.6 \%)$ \\
\hline $25-44$ & $23(44.2 \%)$ & $20(39.2 \%)$ & $43(41.7 \%)$ \\
\hline $45-64$ & $21(40.4 \%)$ & $19(37.3 \%)$ & $40(38.8 \%)$ \\
\hline$\geq 65$ & $1(1.9 \%)$ & $5(9.8 \%)$ & $6(5.8 \%)$ \\
\hline \multicolumn{4}{|l|}{ Marital status*, n (\%) } \\
\hline Married/Cohabiting & $24(46.1 \%)$ & $28(54.9 \%)$ & $52(50.5 \%)$ \\
\hline Living alone & $15(28.8 \%)$ & $12(23.5 \%)$ & $27(26.2 \%)$ \\
\hline Living with parents & $7(13.5 \%)$ & $8(15.7 \%)$ & $15(14.6 \%)$ \\
\hline Other & $3(5.8 \%)$ & $1(2.0 \%)$ & $4(3.9 \%)$ \\
\hline Missing values & 3 & 2 & $5(4.9 \%)$ \\
\hline \multicolumn{4}{|l|}{ Education, n (\%) } \\
\hline No education & $2(3.8 \%)$ & $4(7.8 \%)$ & $6(5.8 \%)$ \\
\hline Primary school & $1(1.9 \%)$ & $3(5.9 \%)$ & $4(3.9 \%)$ \\
\hline Pre-vocational secondary school & $29(55.8 \%)$ & $30(58.8 \%)$ & $59(57.3 \%)$ \\
\hline Secondary School ${ }^{a}$ & $6(11.5 \%)$ & $3(5.9 \%)$ & $9(8.7 \%)$ \\
\hline Higher education ${ }^{\mathrm{b}}$ & $10(19.2 \%)$ & $6(11.8 \%)$ & $16(15.5 \%)$ \\
\hline Other & $2(3.8 \%)$ & $3(5.9 \%)$ & $5(4.9 \%)$ \\
\hline Missing values & 2 & 2 & $4(3.9 \%)$ \\
\hline \multicolumn{4}{|l|}{ Daily life, n (\%) } \\
\hline Study & $6(11.5 \%)$ & $3(5.9 \%)$ & $9(8.7 \%)$ \\
\hline Work & $20(38.5 \%)$ & $11(21.6 \%)$ & $31(30.1 \%)$ \\
\hline Entrepreneur & $4(7.7 \%)$ & 0 & $4(3.9 \%)$ \\
\hline Housewife/houseman & $3(5.8 \%)$ & $5(9.8 \%)$ & $8(7.8 \%)$ \\
\hline Unemployed & $9(17.3 \%)$ & $7(13.7 \%)$ & $16(15.5 \%)$ \\
\hline Incapacitated & $5(9.6 \%)$ & $16(31.4 \%)$ & $21(20.4 \%)$ \\
\hline Retirement or early retirement & $2(3.8 \%)$ & $5(9.8 \%)$ & $7(6.8 \%)$ \\
\hline Other & $1(1.9 \%)$ & $2(3.9 \%)$ & $3(2.9 \%)$ \\
\hline Missing values & $1(1.9 \%)$ & $2(3.9 \%)$ & $3(2.9 \%)$ \\
\hline \multicolumn{4}{|l|}{ Employment $^{* * *}$} \\
\hline Unpaid job / unemployed & 27 (51.9\%) & $37(72.5 \%)$ & $64(62.1 \%)$ \\
\hline Employed & $24(46.2 \%)$ & $11(21.6 \%)$ & $35(34.0 \%)$ \\
\hline Missing & $1(1.9 \%)$ & $3(5.9 \%)$ & $4(3.9 \%)$ \\
\hline $\begin{array}{l}\text { Mean number of seizures } 4 \text { weeks prior baseline } \\
\text { baseline }\end{array}$ & 5.2 (SD: 13.2) & 4.5 (SD: 7.8) & \\
\hline Epilepsy Self-Efficacy Scale score ${ }^{c}$ & 246.5 (SD: 23.3) & 229.0 (SD: 32.3) & \\
\hline \multicolumn{4}{|l|}{ Hospital Anxiety and Depression Scale } \\
\hline Total anxiety score & 6.5 (SD: 3.4) & 7.43(SD: 3.40 & \\
\hline Total depression score & 4.8 (SD:3.2) & $5.24(S D: 3.0)$ & \\
\hline \multicolumn{4}{|l|}{$\begin{array}{l}\text { Medication Adherence monitoring system } \\
\text { (adherence rate) }\end{array}$} \\
\hline 6 months follow-up & $75.7 \%(S D: 32.5)$ & $63.7 \%(S D: 36.6)$ & \\
\hline 12 months follow-up & $71.0 \%$ (SD:36.6) & 12.81 & \\
\hline
\end{tabular}

ancludes: General vocational school, pre-university school and secondary vocational education; ' Includes Professional and academic higher education; ${ }^{\circ}$ Sig. difference between groups at $5 \%$ level. 


\section{Clinical effectiveness}

There was a significant difference at baseline regarding ESES scores ( $p=0.020)$. No significant differences between groups at 6 or 12 months of follow-up were found.

Mean adherence rates over 6 months, as measured with the MEMS, were $63.7 \%$ for the CAU group and $75.9 \%$ for the intervention group, which was not shown to be a significant difference. Adherence decreased slightly over time, resulting in an adherence rate of $71.05 \%$ at 12 months for the intervention group. In total, 18\% of the subjects in CAU group and $4 \%$ of the subjects in the intervention group did not initiate the MEMS cap and were excluded from the analyses (resulting in 94 patients, 44 from the CAU group and 49 from the intervention group). Patients who were lost to follow-up or who did not used the MEMS caps were considered nonadherent. Utility scores derived from the EQ$5 \mathrm{D}-5 \mathrm{~L}$ did not show significant differences at any of the follow-up measurements. Total QALYs at 6 months were 0.41 for both the intervention group and the control group using Dutch tariffs. Although baseline QOLIE-31-P scores differed, the difference was not significant between groups. At 3-month follow-up, the intervention group had higher QOLIE31-P scores compared to CAU, and total disease-specific QALYS, as derived from the QOLIE-31-P, were higher in the intervention group (0.34) compared to the CAU group (0.32) at 6-month follow-up and at 12-month follow-up, but when adjusting for baseline differences, no significant differences were found between groups. Table 5.2 presents a detailed overview of the clinical effectiveness data.

Table 5.2 Mean utility score and quality adjusted life years (QALYS) per group

\begin{tabular}{|c|c|c|c|c|c|c|c|c|}
\hline & \multicolumn{5}{|c|}{ Intervention group ( $\mathrm{N}=52)$} & \multicolumn{3}{|c|}{ Care as usual $(\mathrm{N}=50)$} \\
\hline & BS & FU3M & FU6M & FU12M & BS & $\mathrm{FU} 3 \mathrm{M}$ & FU 6M & FU12M ${ }^{\mathrm{e}}$ \\
\hline Epilepsy self-efficacy scald* & $243.82^{b}$ & 253.16 & 263.16 & 263.03 & $230.42^{b}$ & 244.67 & 252.32 & 252.32 \\
\hline Epilepsy self-efficacy scale_Adj & & & 258.94 & 257.99 & & & 256.63 & 257.46 \\
\hline \multicolumn{9}{|l|}{ Utilities / QOL } \\
\hline Utilities (NL-tariff) & 0.82 & 0.85 & 0.85 & 0.85 & 0.83 & 0.81 & 0.81 & 0.81 \\
\hline Total QALYs (NL-tariff) & & & 0.42 & 0.85 & & & 0.41 & 0.81 \\
\hline Total QALYs (NL-tariff)_Adj ${ }^{\mathrm{a}}$ & & & 0.42 & 0.85 & & & 0.41 & 0.81 \\
\hline Utilities (UK-tariff) & 0.87 & 0.88 & 0.88 & 0.89 & 0.87 & 0.85 & 0.85 & 0.85 \\
\hline Total QALYs (UK-tariff) ${ }^{\mathrm{a}}$ & & & 0.44 & 0.88 & & & 0.43 & 0.85 \\
\hline Total QALYs (UK-tariff)_Adj ${ }^{\mathrm{a}}$ & & & 0.44 & 0.88 & & & 0.43 & 0.85 \\
\hline Visual analogue scale & 75.84 & 78.45 & 79.00 & $80.52^{b}$ & 73.63 & 74.71 & 74.10 & 74.10 \\
\hline QOLIE-31p & 66.35 & $68.18^{b}$ & 68.63 & 69.99 & 63.21 & $63.40^{b}$ & 66.46 & 66.46 \\
\hline Total disease-specific QALYa & & & $0.34^{\mathrm{b}}$ & $0.69^{b}$ & & & $0.32^{b}$ & $0.65^{b}$ \\
\hline $\begin{array}{l}\text { Total disease-specific } \\
\text { QALY_Adj }{ }^{\mathrm{a}}\end{array}$ & & & 0.34 & 0.68 & & & 0.33 & 0.66 \\
\hline
\end{tabular}

\footnotetext{
a Total QALYs are calculated over 6 months (max QALY is 0.5); ${ }^{b}$ Sig. difference between groups at $5 \%$ level; ${ }^{c}$ Disease-specific QALY are calculated over 6 months, based on total QOLIE-31p scores divided by 100 (max QALY is 0.5); ${ }^{d}$ Significance testing based on mean change score at FU3M and FU6M due to baseline differences; ${ }^{\text {e }}$ Observations at 6 months carried forward; BS: baseline; FU: follow-up; 3M: 3 months; 6M: 6 months; QOL: Quality of life; QOLIE-31p: Quality of life in epilepsy 31 patient weighted
} 
Table 5.3 Mean costs and bootstrapped 95\% confidence intervals (CI) after 6 months (end of comparative phase, no baseline adjustment)

\begin{tabular}{|c|c|c|c|c|}
\hline \multirow[b]{2}{*}{$6 F U$} & \multicolumn{2}{|c|}{ Intervention group ( $\mathrm{N}=52)$} & \multicolumn{2}{|c|}{ Care as usual group $(\mathrm{N}=50)$} \\
\hline & & $12 \mathrm{FU}$ & $6 F U$ & $12 \mathrm{FU}$ \\
\hline Intervention costs & 422 & 648 & 95 & 95 \\
\hline \multicolumn{5}{|l|}{ Health care costs } \\
\hline GP visits & 82 & 166 & 66 & 132 \\
\hline Specialist visits & 139 & 494 & 324 & 649 \\
\hline Paramedic visits & 279 & 579 & 183 & 366 \\
\hline Psychologist visits & 62 & 175 & 99 & 198 \\
\hline Emergency department & 108 & 303 & 109 & 217 \\
\hline Hospital stay & 471 & 945 & 138 & 276 \\
\hline Epilepsy center & 43 & 271 & 17 & 33 \\
\hline Daycare & 0 & 19 & 121 & 242 \\
\hline Personal care & 24 & 142 & 0 & 0 \\
\hline Prescribed medication & 92 & 369 & 74 & 148 \\
\hline Total health care costs ${ }^{a}(\mathrm{Cl})$ & $1292(802-1918)$ & $\begin{array}{l}3109(2189- \\
4323)\end{array}$ & $1122(737-1670)$ & $2244(1442-3335)$ \\
\hline \multicolumn{5}{|l|}{ Patient \& family costs } \\
\hline Home care & 88 & 158 & 176 & 352 \\
\hline Medical aids or transport costs & 45 & 64 & 1 & 2 \\
\hline Total patient \& family costs ${ }^{a}(\mathrm{Cl})$ & $132(25-284)$ & $\begin{array}{l}181(46- \\
337)\end{array}$ & 169 & $338(83-639)$ \\
\hline \multicolumn{5}{|l|}{ Productivity } \\
\hline Absenteeism & 811 & 1485 & 182 & 364 \\
\hline Presentism & 1081 & 2219 & 631 & 1262 \\
\hline Unpaid work & 778 & 1713 & 1268 & 2535 \\
\hline Total production losses ${ }^{a}(\mathrm{Cl})$ & 2823 (1896-3877 & $\begin{array}{l}5378 \text { (4007- } \\
6970)\end{array}$ & $\begin{array}{l}2756(1615- \\
4100)\end{array}$ & $5512(3318-7877)$ \\
\hline Total costs ${ }^{*}(\mathrm{Cl})$ & $\begin{array}{l}4491 \text { (3395- } \\
5815)\end{array}$ & $\begin{array}{l}9314(7509- \\
11228)\end{array}$ & $\begin{array}{l}4142(2890- \\
5568)\end{array}$ & $\begin{array}{l}8189 \text { (5661- } \\
10750)\end{array}$ \\
\hline $\begin{array}{l}\text { Total costs including baseline correction } \\
\text { (Cl) }\end{array}$ & $\begin{array}{l}4467(3363- \\
5662)\end{array}$ & $\begin{array}{l}9014(7241- \\
11006)\end{array}$ & $\begin{array}{l}4345(3199- \\
5574)\end{array}$ & $\begin{array}{l}8495(6135- \\
10920)\end{array}$ \\
\hline
\end{tabular}

${ }^{a}$ For the intervention group extrapolated to $\mathrm{N}=52$ based and for $\mathrm{CAU}$ group to $\mathrm{N}=50$ based on multiple imputation

\section{Cost-effectiveness}

The intervention costs were $€ 422$ and $€ 648$ for the intervention group at 6 and 12 months of follow-up and $€ 95$ for the CAU group. The intervention costs for the control group were mainly protocol driven costs attributable to the MEMS bottle. At 6 months, total costs were higher for the intervention group $(€ 4,491)$ compared to the CAU group $(€ 4,142)$. At 12 months, total costs were $€ 9,314$ for the intervention group and $€ 8,189$ for the CAU group. Total costs at 12 months of follow-up adjusted for baseline differences were $€ 9,014$ for the intervention group and $€ 8,495$ for the CAU group (see Table 5.3). 
Looking at the ESES, the combination of effects and costs resulted in an ICER of $€ 54$ per point increase in ESES score at 6 months and $€ 1,105$ per point increase in ESES score at 12 months of follow-up. Concerning adherence, the intervention resulted in an ICER of $€ 88$ per percentage of adherence increase at 6 months. When looking at the QALYS (Dutch tariff), an ICUR of $€$ 8,272 per QALY gain was found at 6-month follow-up and an ICUR of $€ 15,144$ per QALY gained at 12-month follow-up. Results of the bootstrap replications of the ICERs/ICURs are presented in Figure 5.2, which presents the differences in costs and effects between the $\mathrm{MCl}$ and CAU for each bootstrap replication (1,000 times).

The probability that the $\mathrm{MCl}$ is cost-effective given different ceiling ratios, as presented in CEACs, is shown in Figure 5.3. At a willingness to pay $€ 2,000$ per point increase on the ESES score, the probabilities of the $\mathrm{MCl}$ being cost-effective is $69 \%$ and $52 \%$ at 6 and 12 months of follow-up, respectively. In terms of QALYs, if one would be willing to pay $€ 50,000$ per QALY gained, the probability that the $\mathrm{MCl}$ would be cost-effective is $74 \%$ at 6-month follow-up and 76\% at 12-month follow-up (see Fig.5. 3).

\section{Sensitivity analyses}

Disease-specific QALY: the ICURs at 6 and 12 months of follow-up, respectively, were $€$ 15,975 and $€ 36,824$ per disease-specific QALY gained. Applying a healthcare perspective instead of a societal perspective led to ICERs of $€ 214$ and $€ 2,034$ per score improvement on ESES score at 6 and 12 months, respectively. Looking at adherence, an ICER of $€ 65$ per percentage increase in adherence was found at 6 months. In terms of QALYs, the healthcare perspective led to an ICUR of $€ 31,502$ per QALY gained at 6 months of followup and $€ 27,850$ per QALY gained at 12 months of follow-up.

Excluding the costs of the MEMS for the CAU group resulted in an ICER of $€ 93$ per point increase in ESES score at 6 months, $€ 1,546$ per point increase in ESES score at 12month follow-up, and in ICER of $€ 100$ per percentage adherence increase. Excluding the cost of MEMS for the CAU resulted in an ICUR of $€$ 12,901 per QALY gained at 6 months of follow-up, and $€ 20,363$ per QALY gained at 12 months of follow-up.

Using the UK tariffs to value the EQ-5D-5L health states resulted in ICURs of $€$ 9,983 and $€ 16,658$ per QALY gained at 6 and 12 months of follow-up, respectively.

Finally, the analyses were performed without baseline adjustments. This resulted in an ICER of $€ 48$ per score improvement on ESES score at 6 months, an ICER of $€ 105$ per score improvement on ESES score at 12 months, and an ICER of $€ 147$ per percentage increase in adherence. In terms of QALYs, this led to an ICUR of $€ 30,989$ per QALY gained at 6 months of follow-up and $€ 36,585$ per QALY gained at 12 months of follow-up. 


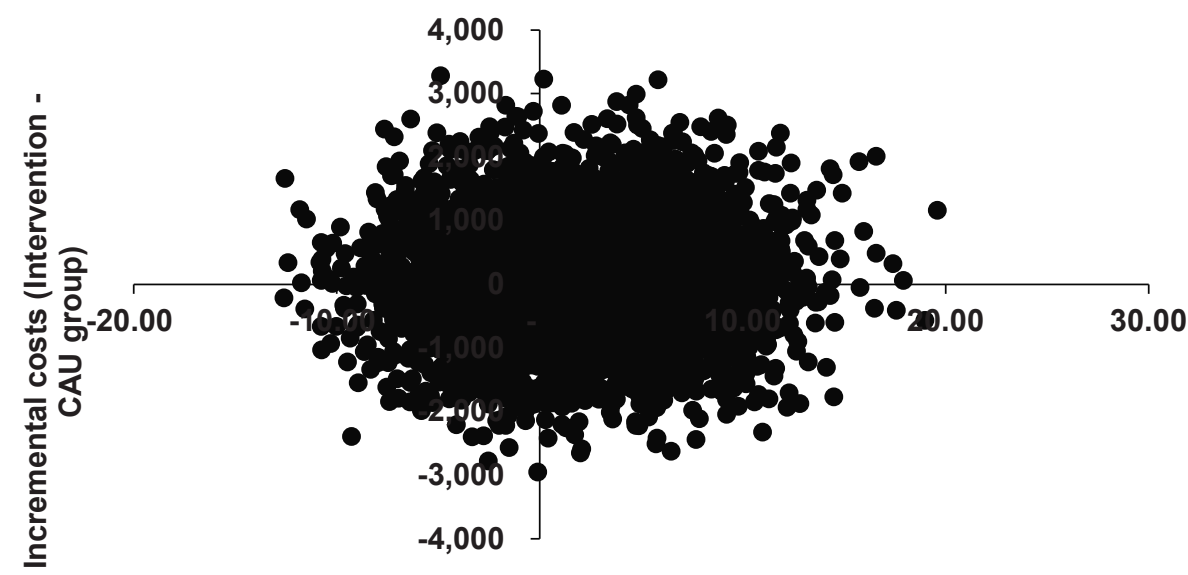

Incremental ESES score (Intervention - CAU group)

Figure 5.2a Cost-effectiveness plane of ESES scores at 6 months' follow-up (costs per point ESES score increase)

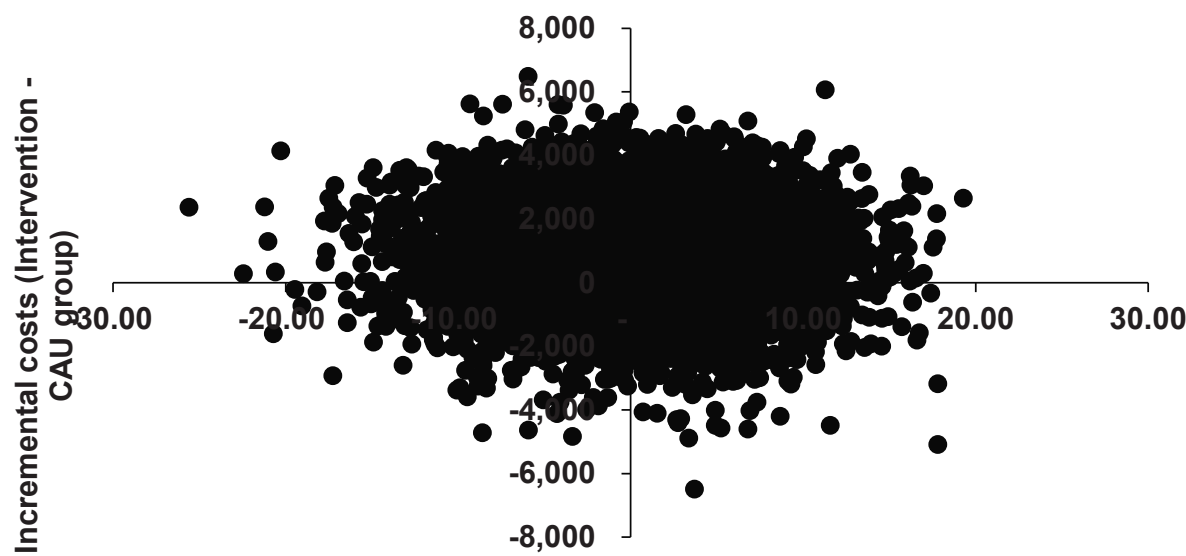

Incremental ESES score (Intervention - CAU group)

Figure 5.2b Cost-effectiveness plane of ESES scores at 12 months' follow-up (costs per point ESES score increase) 


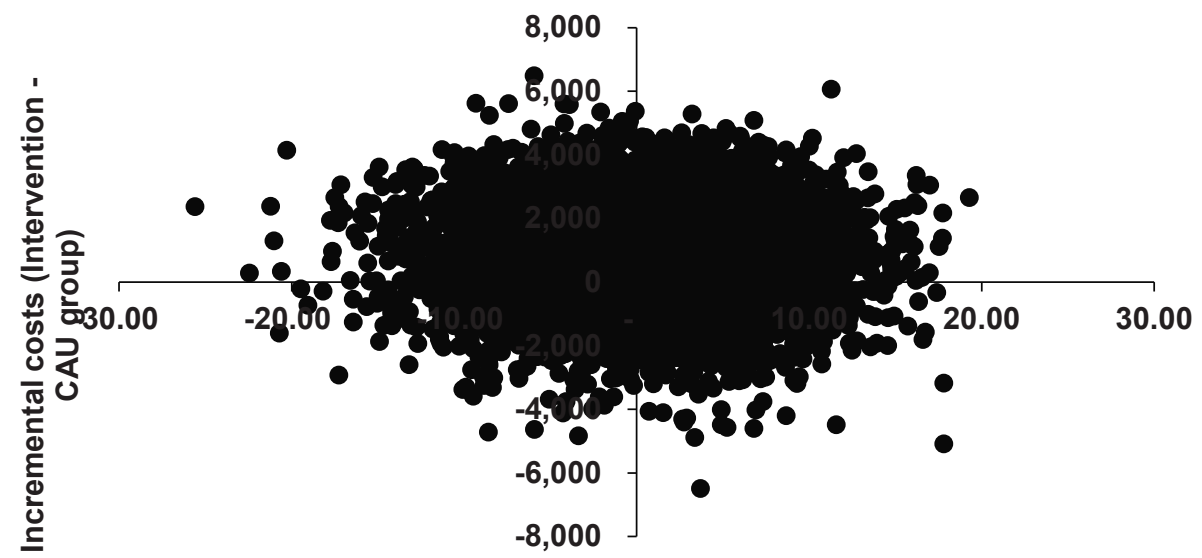

Incremental ESES score (Intervention - CAU group)

Figure 5.2c Cost-effectiveness plane of percentage adherence at 6 months' follow-up (costs per percentage increase in adherence increase)

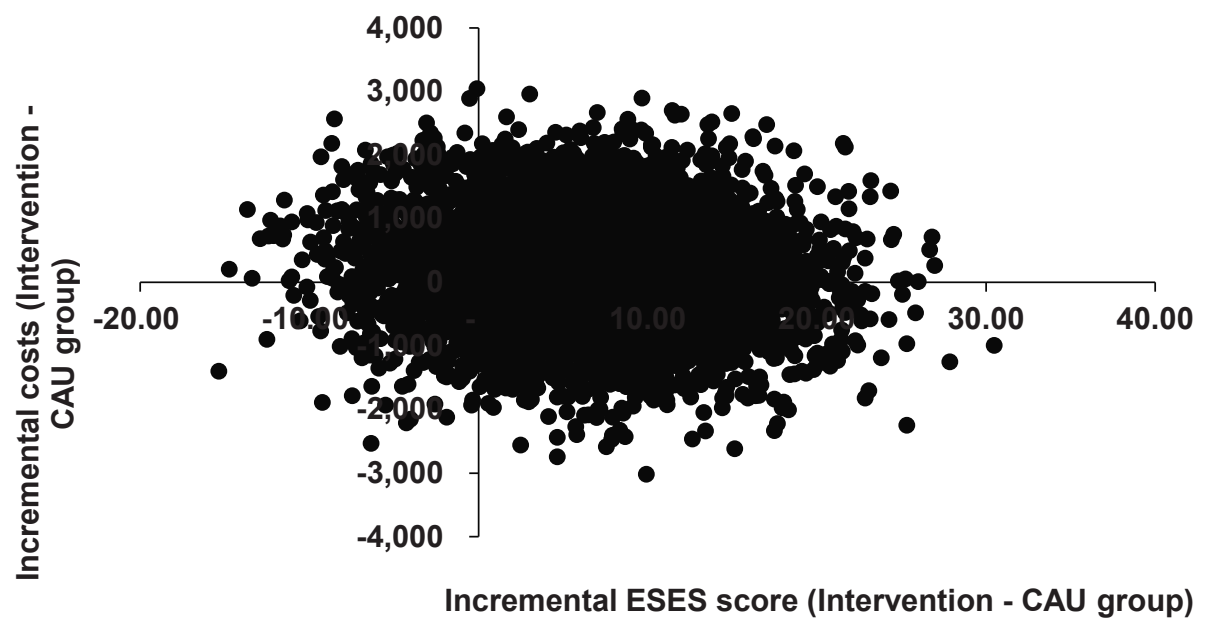

Figure 5.2d Cost-effectiveness plane of QALY scores at 6 months' follow-up (costs per QALY gained) 


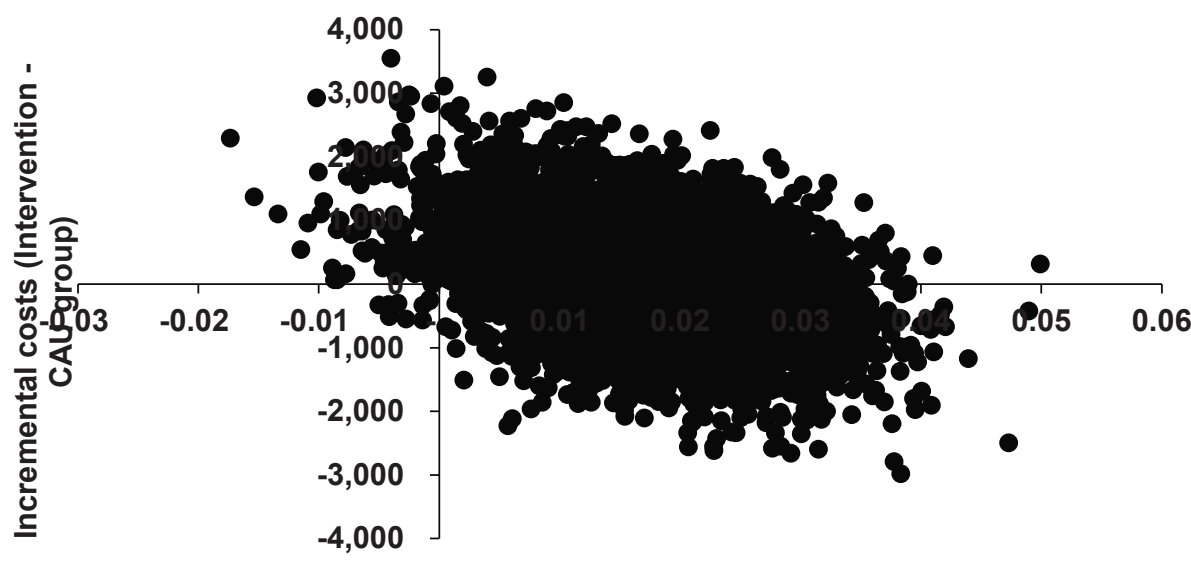

Incremental QALY (Intervention - CAU group)

Figure 5.2e Cost-effectiveness plane of QALY scores at 12 months' follow-up (costs per QALY gained)

A

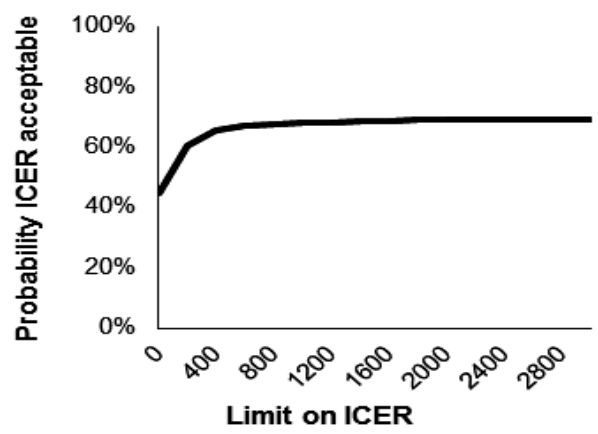

C

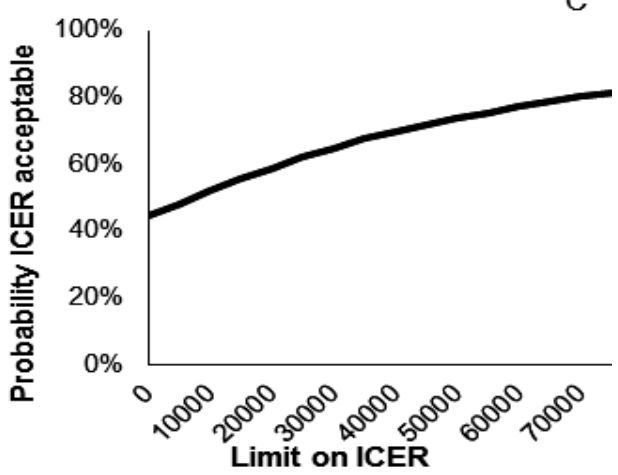

B

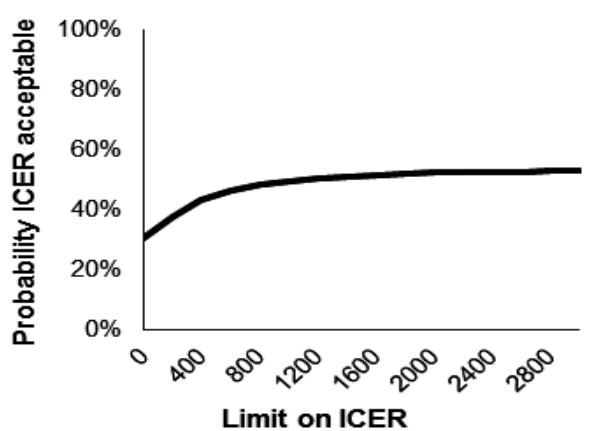

D

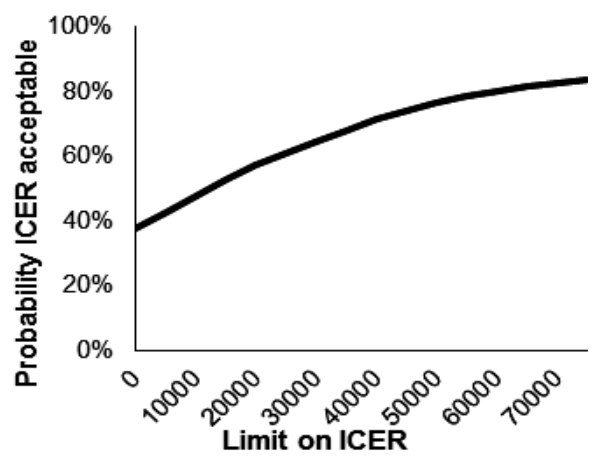




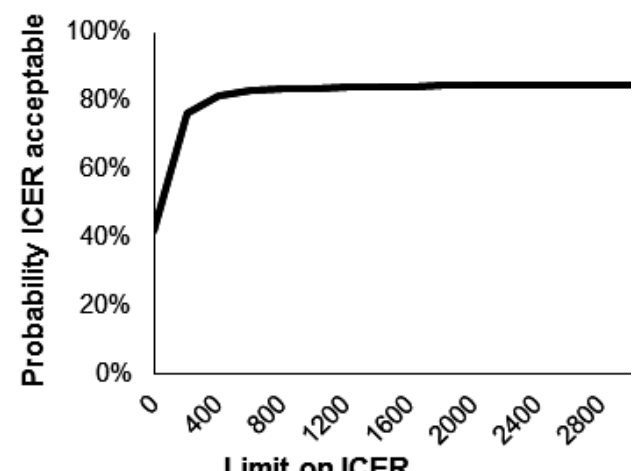

Figure 5.3 A Cost-effectiveness acceptability curve of costs per ESES score improved at 6 months; B cost-effectiveness acceptability curve of costs per ESES score improved at 12 months; C Cost-effectiveness acceptability curve of costs per QALY gained at 6 months; D cost-effectiveness acceptability curve of costs per QALY gained at 12 months; $E$ cost-effectiveness acceptability curve of costs per percentage increase in adherence at 6 months;

\section{DISCUSSION}

Cost-effectiveness estimates appeared to be promising. Unlike, QALYs, there is no willingness to pay threshold known for improvement on the ESES score, which makes it difficult to draw firm conclusions regarding cost-effectiveness. Regarding QALYs, considering a threshold of

$€ 50,000$ per QALY gained, the probability that the $\mathrm{MCl}$ was cost-effective was estimated to be $74 \%$ at 6 months of follow-up and $76 \%$ at 12 months of follow-up. The $\mathrm{MCl}$ did not lead to significantly higher levels of self-efficacy. Furthermore, EQ-5D-5L score did not differ between the two groups. Total costs were higher for the intervention group compared to the control group.

The use of baseline corrections impacted the results as demonstrated by the sensitivity analyses. However, the imbalances in important baseline characteristics made us consider adjusting for them. It has been argued that imbalances in baseline measures could in fact matter without being statistically significant ${ }^{35}$ and that baseline adjustments provide more precise estimates of a treatment effect. ${ }^{34}$ Furthermore, although in contrast to, for example, QALYs, baseline costs are not part of the total costs, one could still expect that patients' baseline costs will influence costs during the study. Hence, it is important to investigate this influence. ${ }^{33}$

The lack of effectiveness of the $\mathrm{MCl}$ on the ESES and EQ-5D-5L may be explained by several factors. Of course, it could be that the intervention is not effective. However, this may be too short-sighted. For example, it is also possible, given the nature of the intervention, that we simply did not capture the effect or that the instruments were not 
sufficiently responsive. The first argument applies mainly to the ESES, as self-efficacy has been shown to increase in other studies investigating self-management programs. ${ }^{36}$ The latter argument applies to the EQ-5D-5L, which has previously been reported not to be responsive in patients with epilepsy. ${ }^{4}$ However, it has been argued that rules of inference are arbitrary and irrelevant to the decisions that clinical and economic evaluations claim to inform. Claxton et al. ${ }^{37}$ argued that, if the objective is to maximize health gain for a given budget, decisions should be based only on the mean net benefits, irrespective of whether differences are statistically significant. This is because one of the mutually exclusive alternatives must be chosen and this decision cannot be postponed. By accepting the arbitrary rules of inference, one will impose costs that can be measured in terms of resources or lost health benefits. ${ }^{37}$

This study is not without its limitations. First, an $\mathrm{MCl}$ tailored to specific patient populations may be more effective. For example, it has been shown that adults with lower education and income may be poorer self-managers than those with higher income and/or educational levels. ${ }^{38}$ Second, because the interventions consist of multiple components, it is difficult to determine which aspect contributed to the effects found in this study. In addition, although the CAU group did not receive feedback on their use, both the CAU and the intervention group received the MEMS, which means CAU deviated from standard care. Third, patients were instructed to use the smartphone application "Eppy" (Epilepsy Foundation, The Netherlands). Because of financial constraints, Eppy was not maintained and updated, which meant that only a minority of the patients were able to use the app. Moreover, it was discontinued in early 2015 and removed from both the App store (Apple Inc., USA) and the Google Play store (Google Inc., USA). Fourth, the sample size of this study was based on a standard deviation for the ESES score of 7 points. However, the actual standard deviation found in this study was $>30$ points, which may have resulted in a decrease in study power. This may be a reason that we did not find statistically significant results. Finally, with regard to the 12-month sensitivity analyses, the CAU group was followed for only 6 months and the results extrapolated to 12 months. This implies that the 6-month follow-up of the CAU group was sufficient to obtain a representative estimation of 12-month follow-up costs.

In conclusion, although there was no statistically significant difference found after baseline adjustments, cost-effectiveness estimates appear to be promising. It has been argued that rules of inference are arbitrary and perhaps less relevant to the decisions that clinical and economic evaluations aim to inform. Further research should attempt to determine which factors of self-management contribute to an increased QoL in patients with epilepsy. 


\section{REFERENCES}

1. Strzelczyk A, Reese JP, Dodel R, Hamer HM. Cost of epilepsy: a systematic review. Pharmacoeconomics 2008; 26: 463-76.

2. (RIVM) HRvVeM. Een gezonder Nederland met meer chronisch zieken. In: RIVM; 2014; http://www.rivm.nl/en/Documents_and_publications/Common_and_Present

/Newsmessages/2014/RIVM_forecasting_study_a_healthier_Netherlands_with_more_people_living_wit h_a_chronic_disease

3. Allers K, Essue BM, Hackett ML, Muhunthan J, Anderson CS, Pickles K, et al. The economic impact of epilepsy: a systematic review. BMC Neurol 2015; 15: 245.

4. de Kinderen RJ, Lambrechts DA, Wijnen BF, Postulart D, Aldenkamp AP, Majoie MH, et al. An economic evaluation of the ketogenic diet versus care as usual in children and adolescents with intractable epilepsy: An interim analysis. Epilepsia 2016; 57: 41-50.

5. Annemans L. Gezondheidseconomie voor niet-economen: Een inleiding tot de begrippen, methoden en valkuilen van de gezondheidseconomische evaluatie: Academia Press; 2007.

6. Williams I, Mclver S, Moore D, Bryan S. The use of economic evaluations in NHS decision-making: a review and empirical investigation. Health Technol Assess 2008; 12: iii, ix-x, 1-175.

7. ZorginstituutNL. Economische evaluatie. In:

https://www.zorginstituutnederland.nl/pakket/werkwijze+pakketbeheer/beoordeling+geneesmiddelen/econo mische+evaluatie

8. Samsonsen C, Reimers A, Brathen G, Helde G, Brodtkorb E. Nonadherence to treatment causing acute hospitalizations in people with epilepsy: an observational, prospective study. Epilepsia 2014; 55: e125-8.

9. Bodenheimer T, Lorig K, Holman H, Grumbach K. Patient self-management of chronic disease in primary care. JAMA 2002; 288: 2469-75.

10. Bourbeau J, Collet JP, Schwartzman K, Ducruet T, Nault D, Bradley C. Economic benefits of selfmanagement education in COPD. Chest 2006; 130: 1704-11.

11. Shegog R, Bamps YA, Patel A, Kakacek J, Escoffery C, Johnson EK, et al. ManagingEpilepsy Well: Emerging e-Tools for epilepsy self-management. Epilepsy Behav 2013; 29: 133-40.

12. Cramer JA, Glassman M, Rienzi V. The relationship between poor medication compliance and seizures. Epilepsy \& Behavior 2002; 3: 338-342.

13. Amir M, Roziner I, Knoll A, Neufeld MY. Self-efficacy and social support as mediators in the relation between disease severity and quality of life in patients with epilepsy. Epilepsia 1999; 40: 216-24.

14. Haynes RB, Ackloo E, Sahota N, McDonald HP, Yao X. Interventions for enhancing medication adherence. Cochrane Database Syst Rev 2008: CD000011.

15. Leenen LA, Wijnen BF, de Kinderen RJ, Majoie MH, van Heugten CM, Evers SM. (Cost)-effectiveness of a multi-component intervention for adults with epilepsy: study protocol of a Dutch randomized controlled trial (ZMILE study). BMC Neurol 2014; 14: 255.

16. Aspinwall LG, Taylor SE. A stitch in time: self-regulation and proactive coping. Psychol Bull 1997; 121: 41736.

17. Thoolen B, de Ridder D, Bensing J, Gorter K, Rutten G. Beyond Good Intentions: the development and evaluation of a proactive self-management course for patients recently diagnosed with type 2 diabetes. Health Educ Res 2008; 23: 53-61.

18. Lorig K, Gonzalez VM, Ritter P. Community-based Spanish language arthritis education program: a randomized trial. Med Care 1999; 37: 957-63.

19. Lorig KR, Holman H. Self-management education: history, definition, outcomes, and mechanisms. Ann Behav Med 2003; 26: 1-7.

20. Lorig KR, Mazonson PD, Holman HR. Evidence suggesting that health education for self-management in patients with chronic arthritis has sustained health benefits while reducing health care costs. Arthritis Rheum 1993; 36: 439-46. 


\section{CHAPTER 5}

21. Dilorio C, Yeager K. The epilepsy self-efficacy scale. Teoksessa: Strickland OL \& Dilorio C (toim.) Measurement of nursing outcomes: Self care and coping, New York 2003: 40-51.

22. Herdman M, Gudex C, Lloyd A, Janssen M, Kind P, Parkin D, et al. Development and preliminary testing of the new five-level version of EQ-5D (EQ-5D-5L). Qual Life Res 2011; 20: 1727-36.

23. Versteegh MM, Vermeulen KM, Evers SM, de Wit GA, Prenger R, Stolk EA. Dutch Tariff for the Five-Level Version of EQ-5D. Value in health 2016.

24. Cramer JA, Van Hammee G, Group NS. Maintenance of improvement in health-related quality of life during long-term treatment with levetiracetam. Epilepsy Behav 2003; 4: 118-23.

25. Devinsky O, Vickrey BG, Cramer J, Perrine K, Hermann B, Meador K, et al. Development of the quality of life in epilepsy inventory. Epilepsia 1995; 36: 1089-104

26. Nederland Z. Richtlijn voor het uitvoeren van economische evaluaties in de gezondheidszorg. Diemen: Zorginstituut Nederland 2015

27. Husereau D, Drummond M, Petrou S, et al. Consolidated health economic evaluation reporting standards (CHEERS) statement. Cost Effand Resour Alloc 2013;11:6.

28. Grittner U, Gmel G, Ripatti S, Bloomfield K, Wicki M. Missing value imputation in longitudinal measures of alcohol consumption. Int J Methods Psychiatr Res 2011; 20: 50-61.

29. Horton NJ, Lipsitz SR. Multiple imputation in practice: comparison of software packages for regression models with missing variables. The American Statistician 2001; 55: 244-254.

30. Drummond MF, Sculpher MJ, Claxton K, Stoddart GL, Torrance GW. Methods for the economic evaluation of health care programmes: Oxford university press; 2015.

33. van Asselt AD, van Mastrigt GA, Dirksen CD, Arntz A, Severens JL, Kessels AG. How to deal with cost differences at baseline. Pharmacoeconomics 2009;27: 519-28.

34. Manca A, Hawkins N, Sculpher MJ. Estimating mean QALYs in trial-based cost-e ffectiveness analysis: the importance of controlling for baseline utility. Health Econ 2005; 14: 487-96.

35. Pocock SJ, Assmann SE, Enos LE, Kasten LE. Subgroup analysis, covariate adjustment and baseline comparisons in clinical trial reporting: current practice and problems. Stat Med 2002; 21: 2917-30.

36. Dilorio C, Bamps Y, Walker ER, Escoffery C. Results of a research study evaluating WebEase, an online epilepsy self-management program. Epilepsy Behav 2011; 22: 469-74.

37. Claxton K. The irrelevance of inference: a decision-making approach to the stochastic evaluation of health care technologies. J Health Econ 1999; 18: 341-64.

38. Escoffery C, Bamps Y, LaFrance WC, Jr., Stoll S, Shegog R, Buelow J, et al. Development of the Adult Epilepsy Self-Management Measurement Instrument (AESMMI). Epilepsy Behav 2015; 50: 172-83. 


\section{SUPPLEMENTARY MATERIAL 5.1:}

\section{Overview of the most important costs categories ZMILE-study}

Supplementary Material 5.1

\begin{tabular}{|c|c|c|}
\hline Care type & $\begin{array}{l}\text { Unit price (in euros; } \\
\text { 2015) }\end{array}$ & Source \\
\hline \multicolumn{3}{|l|}{ Intervention costs (12 months) } \\
\hline $\begin{array}{l}\text { Mean costs course (per } \\
\text { patient) }\end{array}$ & 91.38321668 & $\begin{array}{l}\text { (Calculated from) respective providers or professional } \\
\text { organizations. }\end{array}$ \\
\hline MEMS-cap with LCD & 85.5 & $\begin{array}{l}\text { (Calculated from) respective providers or professional } \\
\text { organizations. }\end{array}$ \\
\hline Feedback every 3 months & s 452.3659195 & $\begin{array}{l}\text { (Calculated from) respective providers or professional } \\
\text { organizations. }\end{array}$ \\
\hline Other & 18.45 & $\begin{array}{l}\text { (Calculated from) respective providers or professional } \\
\text { organizations. }\end{array}$ \\
\hline \multicolumn{3}{|l|}{ Costs CAU group (12 months) } \\
\hline MEMS-cap without LCD & 76.5 & $\begin{array}{l}\text { (Calculated from) respective providers or professional } \\
\text { organizations. }\end{array}$ \\
\hline Other & 18.45 & $\begin{array}{l}\text { (Calculated from) respective providers or professional } \\
\text { organizations. }\end{array}$ \\
\hline \multicolumn{3}{|l|}{ Healthcare costs } \\
\hline GP & 33 & Dutch guidelines for costing studies. \\
\hline Specialists visit & 91 & Dutch guidelines for costing studies. \\
\hline Social worker & 65 & Dutch guidelines for costing studies. \\
\hline Paramedic visits & 33 & Dutch guidelines for costing studies. \\
\hline Physiotherapist visit & 33 & Dutch guidelines for costing studies. \\
\hline Occupational therapist & 33 & Dutch guidelines for costing studies. \\
\hline Speech therapist visit & 30 & Dutch guidelines for costing studies. \\
\hline Dietician visit & 31 & $\begin{array}{l}\text { (Calculated from) respective providers or professional } \\
\text { organizations. }\end{array}$ \\
\hline Psychologist visits & 94 & Dutch guidelines for costing studies. \\
\hline Psychiatrist visit & 94 & Dutch guidelines for costing studies. \\
\hline Company doctor & 125 & $\begin{array}{l}\text { (Calculated from) respective providers or professional } \\
\text { organizations. }\end{array}$ \\
\hline Personal care (hour) & 23 & Dutch guidelines for costing studies. \\
\hline Home care (hour) & 73 & Dutch guidelines for costing studies. \\
\hline $\begin{array}{l}\text { Emergency department } \\
\text { visit }\end{array}$ & 259 & Dutch guidelines for costing studies. \\
\hline Ambulance transport & 515 & Dutch guidelines for costing studies. \\
\hline $\begin{array}{l}\text { Outpatient visit general } \\
\text { hospital }\end{array}$ & 91 & Dutch guidelines for costing studies. \\
\hline Outpatient visit epilepsy & 96.5 & Dutch guidelines for costing studies. \\
\hline $\begin{array}{l}\text { Hospital stay, average } \\
\text { (day) }\end{array}$ & 476 & Dutch guidelines for costing studies. \\
\hline
\end{tabular}




\begin{tabular}{|c|c|c|c|}
\hline & Epilepsy centre & 632 & $\begin{array}{l}\text { (Calculated from) respective providers or professional } \\
\text { organizations. }\end{array}$ \\
\hline & 24h EEG registration & 823.0714692 & $\begin{array}{l}\text { (Calculated from) respective providers or professional } \\
\text { organizations. }\end{array}$ \\
\hline & MRI & 207.3338513 & Dutch guidelines for costing studies. \\
\hline & CT-scan & 129.8352758 & Dutch guidelines for costing studies. \\
\hline & EEG scan & 236.4000697 & $\begin{array}{l}\text { (Calculated from) respective providers or professional } \\
\text { organizations. }\end{array}$ \\
\hline & Medication & Variable & $\underline{\text { Www.medicijnkosten.nl }}$ \\
\hline \multicolumn{4}{|c|}{ Patient and family costs } \\
\hline & Housekeeping (hour) & 23 & Dutch guidelines for costing studies. \\
\hline & Transport (per kilometer) & 0.19 & Dutch guidelines for costing studies. \\
\hline & Medical aids & Variable & $\begin{array}{l}\text { (Calculated from) respective providers or professional } \\
\text { organizations. }\end{array}$ \\
\hline \multicolumn{4}{|l|}{$\begin{array}{l}\text { Other } \\
\text { costs }\end{array}$} \\
\hline & Absenteeism & 34.98 & Dutch guidelines for costing studies. \\
\hline & Presentism & 34.98 & Dutch guidelines for costing studies. \\
\hline & Unpaid work & 14.09 & Dutch guidelines for costing studies. \\
\hline
\end{tabular}


CHAPTER

Effectiveness of a Multi-component Self-Management Intervention for Adults with Epilepsy (ZMILE study): A Randomized Controlled Trial

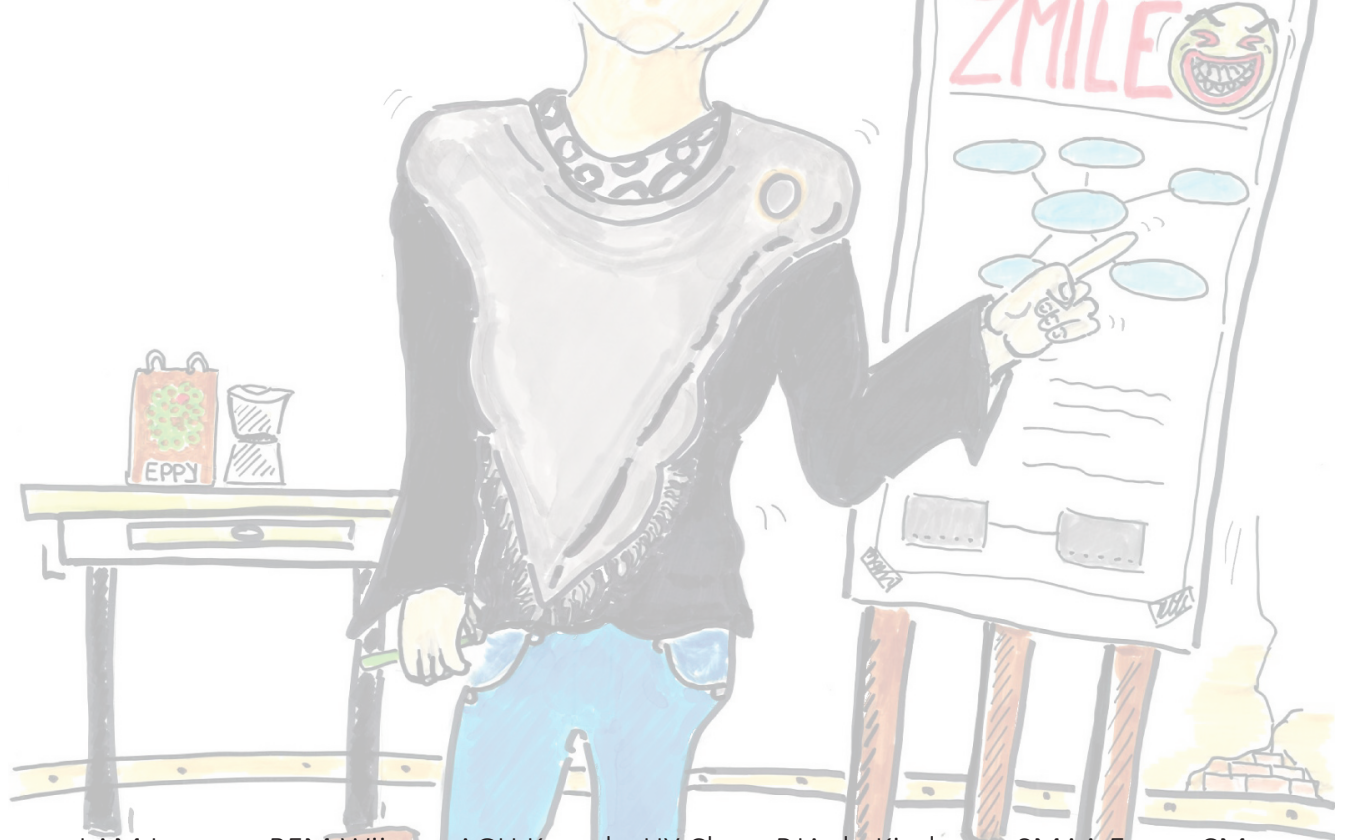

LAM Leenen, BFM Wijnen, AGH Kessels, HY Chan, RJA de Kinderen, SMAA Evers, CM van Heugten, MHJM Majoie

Epilepsy \& Behavior 2018; 80: 259-265 


\section{ABSTRACT}

\section{Background}

The objective of the ZMILE study was to compare the effectiveness of a multicomponent self-management intervention (MCl) with care as usual (CAU) in adult patients with epilepsy (PWE) over a six-month period.

\section{Methods}

Participants (PWE \& relative) were randomized into intervention or CAU groups.

Self-report questionnaires were used to measure disease-specific self-efficacy as the primary outcome measure and general self-efficacy, adherence, seizure severity, emotional functioning, quality of life, proactive coping and side-effects of drugs (AED) as secondary outcome measures. Instruments used at baseline and during a six-month followup period were: disease-specific self-efficacy (Epilepsy Self-Efficacy Scale [ESES]), general self-efficacy (General Self-efficacy [GSES]); adherence (Medication Adherence Scale [MARS] and Medication Event Monitoring System [MEMS]); seizure severity (National Hospital Seizure Severity Scale [NHS3]); emotional well-being (Hospital Anxiety and Depression Scale [HADS]); quality of life (Quality Of Life in Epilepsy [QOLIE-31P]); proactive coping (Utrecht Proactive Coping Competence [UPCC]) and side-effects of Anti-epileptic Drugs [SIDAED]. Multi-level analyses were performed and baseline differences were corrected by inclusion of covariates in the analyses.

\section{Results}

In total, 102 PWE were included in the study, 52 of whom in the intervention group. On the (SIDAED and on three of the quality of life subscales QOLIE-31P, a significant difference was found $(p<0.05)$ in the intervention group. Self-efficacy, however, showed no significant differences between the $\mathrm{MCl}$ and the CAU. None of the other outcome measures showed any significant difference between the two groups.

\section{Significance}

Although we found no statistically significant difference in the primary outcome measure, disease-specific self-efficacy, this $\mathrm{MCl}$ could prove promising, since we found improvement in some domains of quality of life in epilepsy scale and a decrease in AED sideeffects in the $\mathrm{MCl}$ group compared with the CAU group. 


\section{INTRODUCTION}

Having epilepsy is associated with psychological and emotional problems, such as depression and anxiety, which are strongly reflected in a reduced quality of life. ${ }^{1,2}$ Unpredictable seizures are likely to influence daily activities (e.g. employment) of PWE. ${ }^{2}$ Thus, as well as managing their symptoms, PWE and their relatives ${ }^{3}$ must acquire disease-specific knowledge, adhere to treatment and lifestyle regimes, and cope with the psychosocial consequences of the condition. ${ }^{4,5}$

Most PWE use anti-epileptic drugs (AED) and concordance is of great importance for achieving and maintaining positive seizure control. ${ }^{6}$ Concordance refers to the consensual agreement about taking AED that has been established between patient and practitioner. ${ }^{7}$ Poor concordance has been shown to be the most important cause of poorly controlled epilepsy ${ }^{6}$; many PWE seem to be unaware of missed drug intake. ${ }^{8}$ To improve concordance, some self-management programmes focus on the use of e-Health tools (e.g. digital pill dispensers) ${ }^{9}$, although this is not very common in PWE. ${ }^{10}$

One of the options for increasing concordance could be self-management support for PWE (and relatives) using an evidence-based self-management programme, which includes goal-setting, problem-solving, symptom management and shared decision-making. ${ }^{11-13}$

The aim of self-management support is to provide education and supportive interventions to increase skills and confidence in managing health-related problems. ${ }^{12}$ Self-efficacy (i.e. confidence to behave with the intention of reaching a desired goal) is one of the mechanisms responsible for improvement in health outcomes and quality of life, as demonstrated by those attending self-management programmes. ${ }^{4,14,15}$

Proactive coping is helpful in dealing with anticipated challenges in order to reach the desired goal. ${ }^{16}$ The concept of self-management is complex with many different definitions and conceptualizations and, therefore, many forms of support exist. ${ }^{17}$ There is little evidence to prove the effectiveness of self-management programmes within the care of PWE. ${ }^{18} \mathrm{We}$, therefore, developed a multi-component intervention $(\mathrm{MCl})$, consisting of a self-management education programme with e-Health interventions directed at improving self-efficacy, thus, improving the self-management skills of PWE.

The goal of this study (the ZMILE-study) was to evaluate the clinical effectiveness of the $\mathrm{MCl}$. Our primary expectation was that we would find a higher level of disease-specific self-efficacy in the intervention group compared with those who received CAU. Secondarily, we expected to find higher levels of general self-efficacy, adherence (as a proxy for concordance) and proactive coping. We also expected to find a positive change in seizure severity, emotional functioning, quality of life and experienced side-effects of AED, these outcome measurements are recommended outcomes in epilepsy research. ${ }^{19}$ 


\section{METHOD}

\section{Design}

The ZMILE-study was a randomized controlled trial with two parallel groups in which we evaluated the impact of the $\mathrm{MCl}$ in comparison with $\mathrm{CAU}$. The complete study protocol has already been published. ${ }^{20}$ In this paper, we report the clinical effectiveness. Outcome measurements were assessed at baseline (BS) and at three and six months' follow-up (FU3M and FU6M). The primary outcome measure of the study was disease-specific selfefficacy; secondary outcomes measures were: general self-efficacy, adherence, seizure severity, emotional functioning, quality of life, proactive coping and side-effects of AED. Instruments used to assess outcome measurements are shown in table 6.1. The costeffectiveness and the process evaluation of the ZMILE-study are reported elsewhere. ${ }^{21,22}$

Table 6.1: Overview of measurements per time point

\begin{tabular}{|c|c|c|c|c|c|}
\hline Outcomes & Instrument & Range & BS & FU3M & FU6M \\
\hline & & poor - g & & & \\
\hline \multicolumn{6}{|c|}{ Primary outcome measure } \\
\hline Self-efficacy & Epilepsy Self-efficacy Scale (ESES) ${ }^{34}$ & $33-330$ & $x$ & $x$ & $x$ \\
\hline \multicolumn{6}{|c|}{ Secondary outcome measures } \\
\hline General Self-efficacy & Generic Self-efficacy Scale (GSES) ${ }^{35}$ & $10-40$ & $x$ & $x$ & $x$ \\
\hline Adherence & MEMS ${ }^{6}$ & NA & $x$ & $x$ & $x$ \\
\hline Adherence & Medication Adherence Scale (MARS-5) ${ }^{36}$ & $5-25$ & $x$ & $x$ & $x$ \\
\hline Seizure frequency & Questionnaire seizure frequency & - & $x$ & $x$ & $x$ \\
\hline Seizure severity* & $\begin{array}{l}\text { National Hospital Seizure Severity Scale } \\
(\mathrm{NHS} 3)^{37}\end{array}$ & $27-1$ & $x$ & $x$ & $x$ \\
\hline \multirow[t]{3}{*}{ Emotional functioning } & $\begin{array}{l}\text { Hospital Anxiety and Depression Scale } \\
\text { (HADS) }^{4,39}\end{array}$ & $42-0$ & $x$ & $x$ & $x$ \\
\hline & Subscale anxiety & $21-0$ & & & \\
\hline & Subscale depression & $21-0$ & & & \\
\hline Quality of life & $\begin{array}{l}\text { Quality of Life in Epilepsy (QOLIE- } \\
31 P)^{23,38}\end{array}$ & $0-100$ & $x$ & $x$ & $x$ \\
\hline Proactive Coping & $\begin{array}{l}\text { Utrecht Proactive Coping Competence } \\
(\text { UPCC })^{39}\end{array}$ & $21-84$ & $x$ & $x$ & $x$ \\
\hline Side-Effect** & $\begin{array}{l}\text { Side-effects of Anti-Epileptic Drugs } \\
(\text { SIDAED })^{40}\end{array}$ & $138-0$ & $x$ & $x$ & $x$ \\
\hline
\end{tabular}

BS = baseline outcome assessments; FU3M \& FU6M = Follow-up outcome assessments at 3 \& 6 months; ${ }^{*}$ If no seizures had occurred in the past year, a score of 0 was allocated; ${ }^{* *}$ Only the severity of the side-effects was measured.

\section{Participants}

Eligible PWE for this study were adults a 18 or over, living at home, diagnosed with epilepsy and using AED, who the Dutch language and who were willing and able to use e- 
Health devices belonging to the $\mathrm{MCl} .^{20}$ Excluded were PWE who were not able or willing to function in group activities, or when, based on clinical, it was considered that they would not be able to comprehend topics discussed within the $\mathrm{MCl}$ (e.g. PWE with cognitive deficits).

\section{Procedure}

Between March 2014 and December 2015, the Academic Centre for Epileptology recruited PWE during regular attendances at the neurology clinic, via press releases in national epilepsy magazines (Epilepsie, Transmissie) and via social media (Facebook). All potential candidates were informed about the procedure at an initial meeting with one of the researchers. One week later, PWE who wanted to participate were invited for a second visit, asked to sign an informed consent form and were allocated randomly to either the intervention or CAU group.

Baseline measurements (BS) were conducted after randomization. All participants received at baseline the Medication Event Monitoring System (MEMS) and a set of questionnaires. Participants were asked to fill in the questionnaires at home and send them back in a pre-stamped envelope. Prior to the follow-up visits (FU3M, FU6M), the questionnaires were sent by post so that participants could complete them at home. They were collected during the follow-up visits and the MEMS was read (i.e. the number of times the container was opened). The procedure was approved by the Ethics Committee of Maastricht University/Hospital Maastricht, The Netherlands; an overview is presented in Figure 6.1.

\section{Randomization}

To ensure parallel provision of both groups (intervention \& CAU), two equal cohorts of PWEs were needed at the moment of randomization. PWE were assigned to the intervention group or the CAU group by means of block randomization. Instead of the intended blocks of 10 PWE, we also used blocks of six, eight or 10 PWE for practical reasons. An assistant, not involved in the treatment nor in the trial, executed the procedure using a randomization programme (www.randomization.com). The randomization scheme was distributed to the researcher in sealed envelopes during the first visit, prior to BS. 


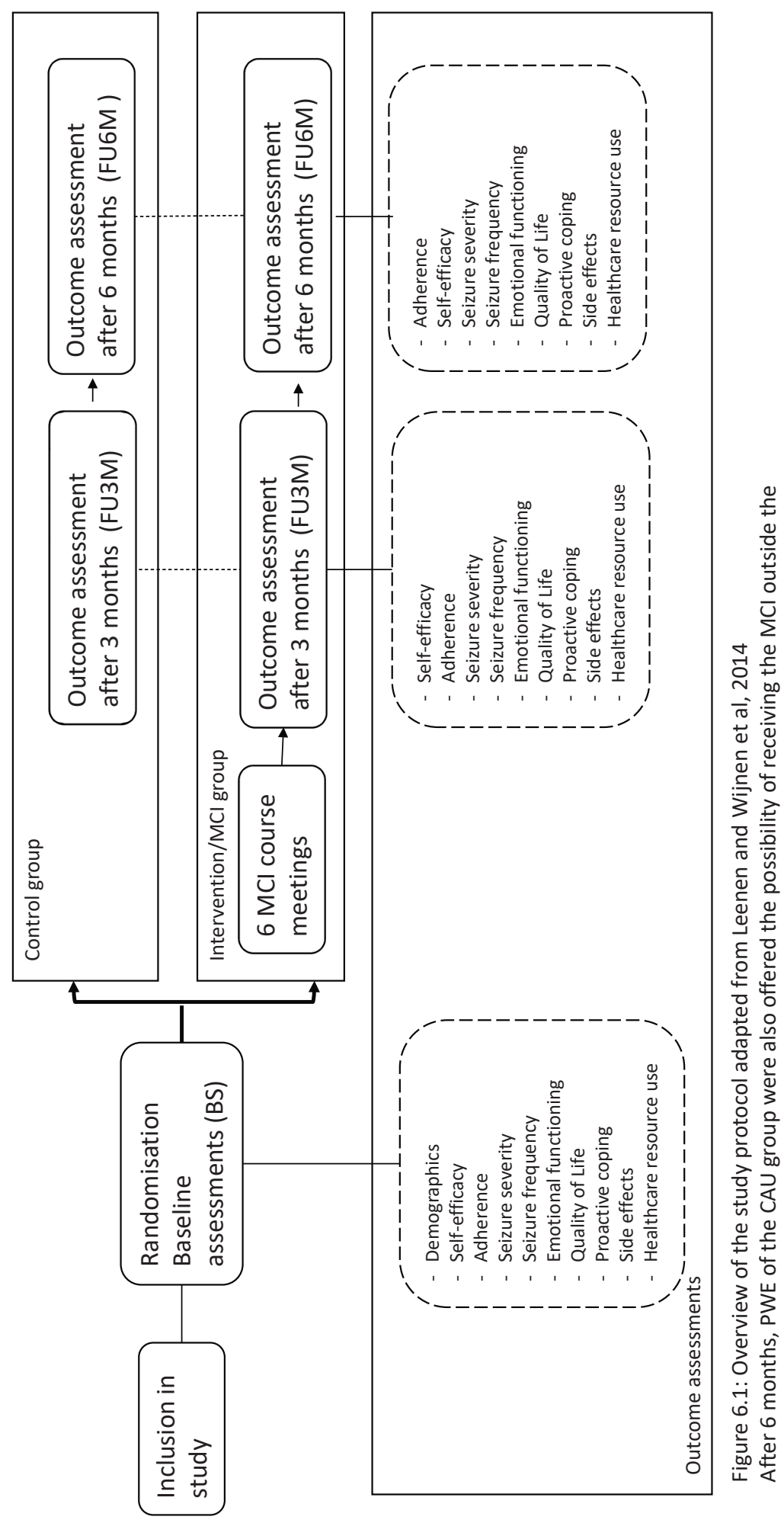




\section{Multi-component intervention}

The $\mathrm{MCl}$ consisted of five weekly group sessions of each, followed by a two-hour booster session after three weeks. The groups comprised three to five PWE with possibly a relative, if present and willing to participate. Sessions led by two nurse practitioners were conducted at several locations in the southern part of the Netherlands (Heeze, Maastricht and Nijmegen). All group sessions consisted of two components: education and practicing goal-setting skills. In the educational part, participants were sharing and discussing strategies about three topics: 1 ) self-monitoring and self-monitoring using (e-Health) tools; 2 ) risk-evaluation and management; and 3) shared decision-making/concordance. The goalsetting component of the intervention is based on Aspinwall and Taylors ${ }^{16}$ five stages of proactive coping, namely: resource accumulation; recognition of potential stressors; initial appraisal; preliminary coping efforts; and elicitation and use of feedback concerning initial efforts. The two eHealth tools used for self-monitoring were a Medication Event Monitoring System (MEMS, Aardex) and a smartphone application 'Eppy' (Epilepsy Foundation, the Netherlands). MEMS are electronic caps with an LCD screen, fitting standard pill bottles, that register the date and time the bottle was opened. These data were stored in an internet-based database end presented in simple plots. Additionally, the LCD-screen provides the user with direct feedback on how many times the bottle had been opened that day. Feedback on medication adherence, as collected by MEMS, was given to the intervention group during the group sessions and after three and six months' follow-up by a nurse practitioner. 'Eppy' is an application that could be downloaded free to a smartphone, giving PWE the opportunity to keep, for instance, a digital seizure diary or to set reminders for taking medication. Data gathered with the Eppy could be synchronized to a website. Access to this website could be granted to healthcare professionals, by the PWE.A more detailed description of the intervention has been published previously. ${ }^{20,22}$

\section{Care as usual}

The control group received unrestricted CAU. Since the ZMILE-study was a pragmatic trial, the CAU-group did not follow a standardized protocol, thus the content of CAU might be variable across PWE, but is expected to be in agreement with standard epilepsy guidelines (http://epilepsie.neurologie.nl). In order to measure adherence, the CAU group also received the MEMS, but without the LCD screen and without the feedback during the visits.

\section{Outcome measurements}

Table 6.1 presents the questionnaires included in the study. These self-report questionnaires were used to measure disease-specific self-efficacy as the primary outcome measure and general self-efficacy, adherence, seizure severity, emotional functioning, quality 
of life, proactive coping and side-effects of AED as secondary outcome measurements. ${ }^{23}$, At baseline, sociodemographic and a limited number of medical variables, such as year of first seizure, were recorded in a patient-reported questionnaire. More details about the administered measurement can be found in the study protocol. ${ }^{20}$

The EuroQol-5D-5L was part of the cost-effectiveness study and, therefore, not included in the present study.

\section{Sample size \& Analyses}

The required sample size for this study was estimated to be 100 persons (i.e. 50 interventions and $50 \mathrm{CAU}) .{ }^{20} \mathrm{All}$ statistical procedures were performed using IBM SPSS statistics version 24 for MacOS, and were based on the intention-to-treat principle. The baseline differences between the $\mathrm{MCI}$ group and CAU group were analysed using independent $\mathrm{t}$ tests or chi-square tests when appropriate. To compare adherence rates, Wilcoxon rank sum tests were used. The first step in handling missing data was to follow the scoring manual of the ESES and HADS questionnaire ${ }^{24,25}$, which prescribes using mean values for handling missing data at item level. SPSS multiple imputations (five times) were used to manage complete data missing from the ESES and HADS, as well as for data missing from other questionnaires. analyses were performed with outcome measurements (BS, FU3M, and FU6M) as within-subjects' factor and group (intervention or CAU) as between subjects' factors to account for the nested structure (e.g., repeated measures) of the data. Baseline differences were corrected by inclusion of covariates in the analyses. Random intercept was used to account for the difference between PWE. A 2-sided significance level of 0.05 was used.26 PWE were excluded if they ding not initiate their MEMS caps.

\section{RESULTS}

\section{Participants}

Figure 6.2 presents a flowchart showing the path taken by the participants. Overall, 102 PWE took part in the study, of whom 86 completed the trial. All assessments were performed within the schedule of 3 months ( \pm 2 weeks). Although depression is a common comorbidity associate with epilepsy, in this sample, average scores on the HADS did not exceed 6.9 for both anxiety and depression during follow-up. These scores are not above the frequently used cut-off point of 8 to define the presence of depression/anxiety. ${ }^{27} \mathrm{Ta}$ ble 6.2 shows the baseline characteristics of the study population. ${ }^{28} \mathrm{At}$ baseline, the intervention group had significantly more PWE who were employed (24/50) than the CAU group (11/48) $(p=.02)$. 


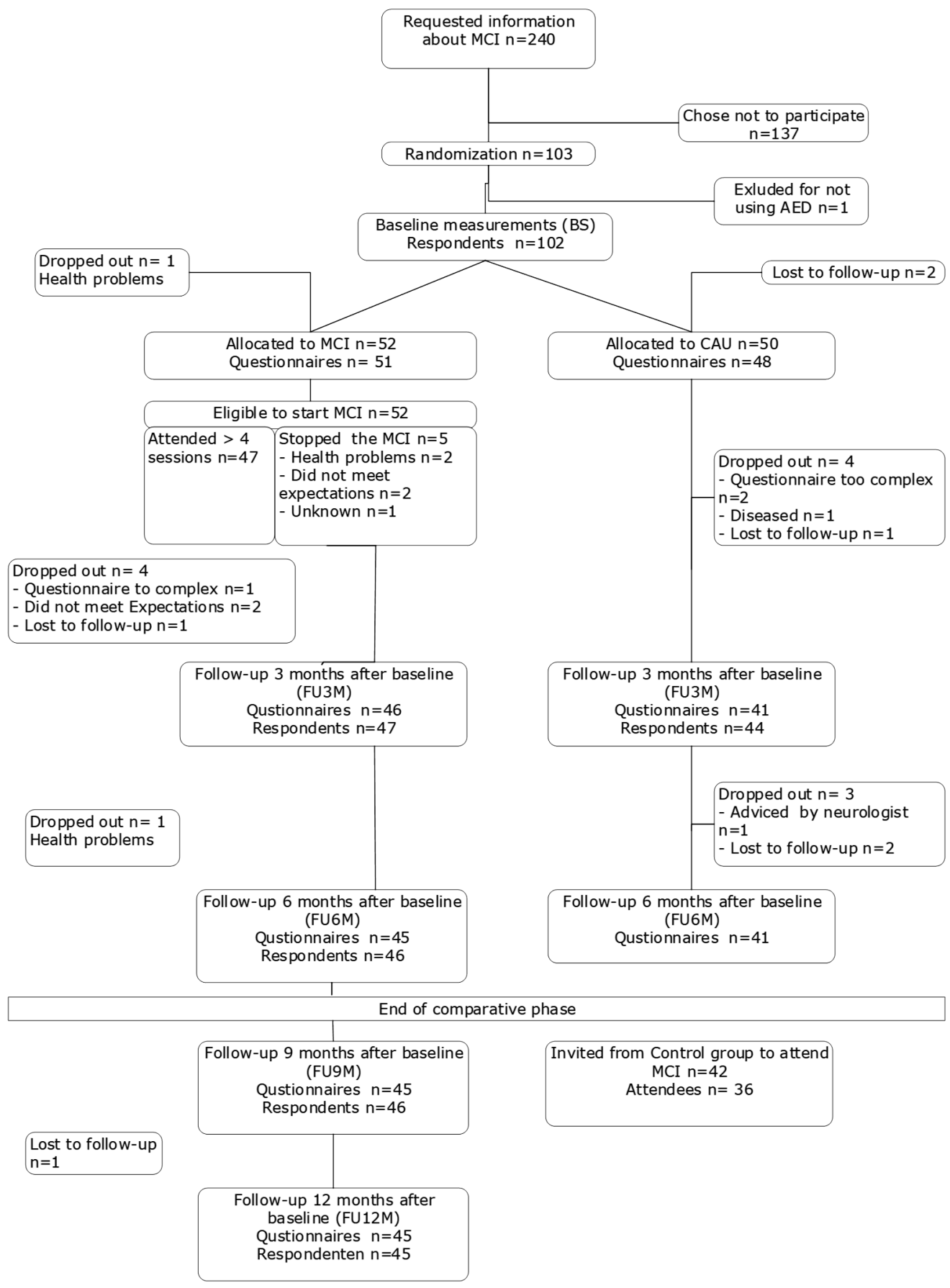

Figure 6.2: Flowchart of participants through the study 
Table 6.2 (Baseline) characteristics of the population

\begin{tabular}{|c|c|c|c|}
\hline Characteristics & $\begin{array}{l}\text { Intervention } \\
\text { group }(n=52)\end{array}$ & $\begin{array}{l}\text { CAU group } \\
(n=50)\end{array}$ & Total $(n=102)$ \\
\hline \multicolumn{4}{|l|}{ Gender, n (\%) } \\
\hline Male & $28(53.9 \%)$ & $22(44.0 \%)$ & $50(49.0 \%)$ \\
\hline Female & $24(46.2 \%)$ & $28(56.0 \%)$ & $52(51.0 \%)$ \\
\hline Age in years, mean (SD) & $40.0(13.1)$ & $43.5(15.4)$ & $41.7(14.7)$ \\
\hline \multicolumn{4}{|l|}{ Age group, n (\%) } \\
\hline $18-24$ & $7(13.5 \%)$ & $7(14.0 \%)$ & $14(13.7 \%)$ \\
\hline $25-44$ & $23(44.2 \%)$ & $20(40.0 \%)$ & $43(42.2 \%)$ \\
\hline $45-64$ & $21(40.4 \%)$ & $18(36.0 \%)$ & $39(38.2 \%)$ \\
\hline$\geq 65$ & $1(1.9 \%)$ & $5(10.0 \%)$ & $6(5.9 \%)$ \\
\hline \multicolumn{4}{|l|}{ Marital status, n (\%) } \\
\hline Married/ living with partner & $24(46.1 \%)$ & $28(56.0 \%)$ & $52(51.0 \%)$ \\
\hline Living alone & $15(28.8 \%)$ & $11(22.0 \%)$ & $26(25.5 \%)$ \\
\hline Living with parents & $7(13.5 \%)$ & $8(16.0 \%)$ & $15(14.7 \%)$ \\
\hline Other & $3(5.8 \%)$ & $1(2.0 \%)$ & $4(3.9 \%)$ \\
\hline Missing values & $3(5.8 \%)$ & $2(4.0 \%)$ & $5(4.9 \%)$ \\
\hline \multicolumn{4}{|l|}{ Highest level of education, $n(\%)$} \\
\hline No education & $2(3.8 \%)$ & $4(8.0 \%)$ & $6(5.9 \%)$ \\
\hline Primary school & $1(1.9 \%)$ & $3(6.0 \%)$ & $4(3.9 \%)$ \\
\hline Pre-vocational secondary school & $29(55.8 \%)$ & $30(60.0 \%)$ & $59(57.8 \%)$ \\
\hline Secondary School ${ }^{\mathrm{a}}$ & $6(11.5 \%)$ & $3(6.0 \%)$ & $9(8.8 \%)$ \\
\hline Higher education ${ }^{b}$ & $10(19.2 \%)$ & $5(10.0 \%)$ & 15 (14.7\%) \\
\hline Other & $2(3.8 \%)$ & $3(6.0 \%)$ & $5(4.9 \%)$ \\
\hline Missing values & $2(3.8 \%)$ & $2(4 \%)$ & $4(3.9 \%)$ \\
\hline \multicolumn{4}{|l|}{ Daily life, n (\%) } \\
\hline Study & $6(11.5 \%)$ & $3(6.0 \%)$ & $9(8.8 \%)$ \\
\hline Work & $20(38.5 \%)$ & $11(22.0 \%)$ & $31(30.4 \%)$ \\
\hline Entrepreneur & $4(7.7 \%)$ & 0 & $4(3.9 \%)$ \\
\hline Housewife/househusband & $3(5.8 \%)$ & $5(10.0 \%)$ & $8(7.8 \%)$ \\
\hline Unemployed & $9(17.3 \%)$ & $7(14.0 \%)$ & $16(15.7 \%)$ \\
\hline Incapacitated & $5(9.6 \%)$ & $16(32.0 \%)$ & $21(20.6 \%)$ \\
\hline Retirement or early retirement & $2(3.8 \%)$ & $4(8.0 \%)$ & $6(5.9 \%)$ \\
\hline Other & $2(3.8 \%)$ & $2(4.0 \%)$ & $4(3.9 \%)$ \\
\hline Missing values & $1(1.9 \%)$ & $2(4.0 \%)$ & $3(2.9 \%)$ \\
\hline \multicolumn{4}{|l|}{ Employment ${ }^{\mathrm{c}}$} \\
\hline Unpaid job / unemployed & 27 (51.9\%) & $36(72.0 \%)$ & $63(61.8 \%)$ \\
\hline Employed & $24(46.2 \%)$ & $11(22.0 \%)$ & $35(34.3 \%)$ \\
\hline Missing values & $1(1.9 \%)$ & $3(6.0 \%)$ & $4(3.9 \%)$ \\
\hline \multicolumn{4}{|l|}{ Medication at baseline, Mean (SD) } \\
\hline Total number of medications & $3.5(1.7)$ & $4.2(2.6)$ & $3.8(2.2)$ \\
\hline AED & $1.9(0.9)$ & $2.1(1.0)$ & $2.1(1.0)$ \\
\hline Missing & 3 & 3 & \\
\hline Seizure frequency at baseline Mean (SD) & $4.5(11.0)$ & $5.8(11.3)$ & \\
\hline Missing & 3 & 4 & \\
\hline Years since first seizure Mean (SD) & $20.3(14.2)$ & $19.9(15.8)$ & \\
\hline Missing & 2 & 7 & \\
\hline
\end{tabular}

${ }^{a}$ Includes: General vocational school, pre-university school and secondary vocational education; ${ }^{\mathrm{b}}$ Includes: Professional and academic higher education; ${ }^{c}$ Sig. difference between groups at $5 \%$ level 
Table 6.3: Overview of Clinical outcomes and significance testing

\begin{tabular}{|c|c|c|c|c|c|c|}
\hline \multirow[t]{2}{*}{ Measures } & \multicolumn{3}{|c|}{ Intervention group $\mathrm{N}=52$} & \multicolumn{3}{|c|}{ Care as usual $\mathrm{N}=50$ ) } \\
\hline & $\mathrm{BS}^{\mathrm{a}}$ & FU3M & FU6M & BS & FU 3M & FU 6M \\
\hline \multicolumn{7}{|l|}{ Primary, Mean (SD) } \\
\hline \multicolumn{7}{|l|}{ Self-efficacy } \\
\hline \multirow[t]{2}{*}{ Epilepsy self-efficacy scale } & $243.8^{a}$ & 253.2 & 263.2 & $230.4^{a}$ & 244.7 & 252.3 \\
\hline & $(23.3)$ & $(30.4)$ & $(26.3)$ & $(32.3)$ & $(32.1)$ & $(32.8)$ \\
\hline \multicolumn{7}{|l|}{ Secondary, Mean (SD) } \\
\hline \multicolumn{7}{|l|}{ Self-efficacy } \\
\hline \multirow[t]{2}{*}{ General Self-Efficacy Scale } & 29.4 & 31.5 & 31.7 & 27.9 & 29.3 & 30.0 \\
\hline & $(5.8)$ & $(5.2)$ & $(4.6)$ & $(5.6)$ & $(5.1)$ & $(5.4)$ \\
\hline \multicolumn{7}{|l|}{ Adherence } \\
\hline \multirow[t]{2}{*}{ Medication Event Monitoring System ${ }^{c}$ (MEMS) } & & & $75.9 \%$ & & & $63.7 \%$ \\
\hline & & & $(32.5)$ & & & $(36.6)$ \\
\hline \multirow[t]{2}{*}{ Medication Adherence Rating Scale (MARS) } & 23.4 & 23.6 & 23.9 & 23.8 & 23.9 & 23.7 \\
\hline & $(2.0)$ & $(1.4)$ & $(0.9)$ & $(1.1)$ & $(1.1)$ & $(1.3)$ \\
\hline \multicolumn{7}{|l|}{ Seizure Severity } \\
\hline \multirow[t]{2}{*}{ National Hospital Seizure Severity Scale (NHS3) } & 6.7 & 7.3 & 6.2 & 9.0 & 8.4 & 8.7 \\
\hline & $(7.5)$ & $(8.1)$ & $(7.3)$ & $(9.6)$ & $(9.3)$ & $(10.0)$ \\
\hline \multicolumn{7}{|l|}{ Depression/Anxiety } \\
\hline \multirow[t]{2}{*}{ Hospital Anxiety and Depression Scale (HADS)-Total } & 11.3 & 11.0 & 9.8 & 12.7 & 11.5 & 11.6 \\
\hline & $(6.1)$ & $(5.2)$ & $(5.0)$ & $(5.9)$ & $(6.2)$ & $(6.2)$ \\
\hline \multirow[t]{2}{*}{ HADS -Depression } & 5.3 & 5.7 & 5.2 & 5.7 & 5.5 & 5.5 \\
\hline & $(2.6)$ & $(2.7)$ & $(2.6)$ & $(2.3)$ & $(2.6)$ & $(2.8)$ \\
\hline \multirow[t]{2}{*}{ HADS-Anxiety } & 5.9 & 5.2 & 4.7 & 6.9 & 6.1 & 6.0 \\
\hline & $(4.3)$ & $(3.5)$ & $(3.5)$ & $(4.3)$ & $(4.2)$ & $(4.2)$ \\
\hline \multicolumn{7}{|l|}{ Quality of life } \\
\hline \multirow[t]{2}{*}{ Quality of life in epilepsy-31(QOLIE-31-P) -Total } & 66.44 & 69.0 & 69.5 & 62.9 & 62.4 & 65.4 \\
\hline & $(12.3)$ & $(11.3)$ & $(10.8)$ & $(12.4)$ & $(14.8)$ & $(14.4)$ \\
\hline \multicolumn{7}{|l|}{ Subscales } \\
\hline \multirow[t]{2}{*}{ QOLIE-31-P -Energy } & 41.4 & 46.3 & 47.8 & 37.8 & 42.1 & 40.2 \\
\hline & $(23.8)$ & $(23.4)$ & $(22.0)$ & $(22.1)$ & $(26.1)$ & $(23.7)$ \\
\hline \multirow[t]{2}{*}{ QOLIE-31-P -Mood ${ }^{\mathrm{b}}$} & 51.7 & 53.7 & 62.0 & 45.4 & 47.8 & 49.0 \\
\hline & $(23.8)$ & $(28.4)$ & $(23.1)$ & $(25.5)$ & $(29.5)$ & $(27.9)$ \\
\hline \multirow[t]{2}{*}{ QOLIE-31-P -Daily Activities ${ }^{\text {b }}$} & 39.1 & 49.1 & 55.5 & 36.4 & 40.2 & 39.7 \\
\hline & $(26.9)$ & $(27.4)$ & $(29.1)$ & $(25.1)$ & $(26.7)$ & (25.9) \\
\hline \multirow[t]{2}{*}{ QOLIE-31-P -Cognition } & 35.4 & 50.2 & 44.8 & 32.0 & 36.5 & 39.7 \\
\hline & $(23.9)$ & $(27.6)$ & $(23.6)$ & $(28.0)$ & $(30.2)$ & $(25.9)$ \\
\hline QOLIE-31-P -Medication Effects & 51.6 & 56.4 & 57.9 & 43.2 & 51.9 & 45.2 \\
\hline & $(30.6)$ & $(25.7)$ & $(27.6)$ & $(27.2)$ & $(29.0)$ & $(25.2)$ \\
\hline QOLIE-31-P -Seizure Worry & 47.5 & 54.0 & 53.9 & 39.3 & 43.9 & 42.8 \\
\hline & $(31.2)$ & $(28.3)$ & $(26.4)$ & $(26.8)$ & $(29.8)$ & $(32.7)$ \\
\hline QOLIE-31-P -Overall quality of life & 51.1 & 54.4 & 58.8 & 44.8 & 48.0 & 46.7 \\
\hline
\end{tabular}




\begin{tabular}{|c|c|c|c|c|c|c|}
\hline \multirow[t]{2}{*}{ Measures } & \multicolumn{3}{|c|}{ Intervention group $\mathrm{N}=52$} & \multicolumn{3}{|c|}{ Care as usual $\mathrm{N}=50$ ) } \\
\hline & $\mathrm{BS}^{\mathrm{a}}$ & FU3M & FU6M & $\mathrm{BS}$ & FU 3M & FU 6M \\
\hline & $(22.8)$ & $(24.3)$ & $(22.4)$ & $(23.2)$ & $(27.7)$ & $(27.2)$ \\
\hline \multirow[t]{2}{*}{ QOLIE-31-P -Distress ${ }^{b, c}$} & 462.8 & 510.4 & 534.6 & 423.7 & 457.1 & 442.9 \\
\hline & (128.9) & (129.9) & $(101.6)$ & $(142.2)$ & (148.9) & (156.0) \\
\hline \multicolumn{7}{|l|}{ Proactive coping } \\
\hline \multirow[t]{2}{*}{ Utrecht Proactive Coping Competence (UPCC) } & 62.6 & 62.5 & 64.0 & 59.7 & 60.3 & 61.1 \\
\hline & $(11.1)$ & $(8.5)$ & (9.0) & $(6.7)$ & (7.9) & $(7.5)$ \\
\hline \multicolumn{7}{|l|}{ Side-effects } \\
\hline \multirow[t]{2}{*}{ Side-effects of anti-epileptic drugs (SIDAED) ${ }^{b}$} & 26.9 & 23.1 & 19.1 & 27.1 & 26.2 & 25.5 \\
\hline & $(21.6)$ & $(18.7)$ & (15.3) & $(20.3)$ & $(17.6)$ & $(19.1)$ \\
\hline
\end{tabular}

${ }^{1}$ If no seizures had occurred in the past year, a score of 0 was allocated. ${ }^{2}$ Only the severity of the side-effects was measured; ${ }^{\mathrm{a} B S}=$ baseline outcome assessments; FU3M \& FU6M, = Follow-up outcome assessments at 3 and 6 months; ${ }^{b}$ Significant at $5 \%$ level; ${ }^{c}$ Distress scores are expressed on a scale from $0-100$ (percentages) for every subscale and are used to weight total (sub)scores.

\section{Clinical effect of the intervention}

Table 6.3 shows the effects of the intervention. At baseline, the ESES scores of the intervention group were significantly higher than that of the CAU group $(p=0.02)$.

The primary outcome disease-specific self-efficacy showed no significant differences between the $\mathrm{MCl}$ and $\mathrm{CAU}$ group ( $\mathrm{p}>0.05$ ).

In total, $49 / 52$ of the intervention and $44 / 50$ of the CAU were included in the analysis. PWE lost to follow-up or who did not use the cap at all, were considered non-adherent. Adherence rates of those included in the MEMS analysis over 6 months were $63.7 \%$ for the CAU group and $75.9 \%$ for the intervention group; the difference was not significant.

There was a significant difference on the side-effect scale SIDAED ( $p=0.04)$, and on three of the subscales of the QOLIE-31P. Subscales 'Emotional wellbeing' ( $p=0.01)$, 'Social functioning' ( $p=0.001)$ and 'Distress' $(p=0.01)$ had a significantly better result in the intervention group. None of the other outcomes, such as quality of life (QOLIE-31P), adherence (MARS), emotional well-being (HADS), seizure severity (NHS3), and proactive coping (UPCC) showed a significant difference between the two groups.

\section{DISCUSSION}

The aim of this study was to evaluate the clinical effectiveness of the $\mathrm{MCl}$ on diseasespecific self-efficacy (primary outcome) and on adherence, seizure severity, side-effects of AED, emotional functioning, proactive coping, and quality of life (secondary outcomes). We found higher levels of disease specific self-efficacy in both groups over time, but no significant differences in disease-specific self-efficacy between the intervention and CAU group. On the secondary outcomes, we found significant differences on side-effects and 
on some subscales of quality of life, but not on the other outcomes i.e. seizure severity, proactive coping and depression/anxiety.

Not finding a significant difference in (disease-specific) self-efficacy is in contrast to other studies on self-management interventions in epilepsy ${ }^{28}$ or other chronic conditions. ${ }^{4,11}$

In our study, participants showed a high level of disease-specific self-efficacy at baseline. Only 6/102 scored in the bottom half of the scale and 36/102 in the fourth quartile which could reflect a ceiling effect, leaving little room for improvement. ${ }^{29}$ The fact that our participants were volunteers, may have contributed to this ceiling effect. ${ }^{30}$

Risdale et al (2017) ${ }^{31}$ recently found that self-mastery, adherence, depression and anxiety are all associated with quality of life in PWE. It is possible that self-mastery did not improve in our study, but we did not measure this concept, although we used the same secondary outcomes; i.e. adherence, depression and anxiety and quality of life. ${ }^{31}$ Probably our understanding of how the different outcomes interact with each other is at this moment insufficient.

In addition, we did not assess changes in self-management behaviour, such as goalattainment, so it remains unclear which components contributed to the potential effect of the intervention; this is not uncommon in complex interventions. ${ }^{32}$ Furthermore, the CAU was not standardized and there were some additional attributions MEMS and visits). This may have diminished the contrast between the interventions and may, therefore, have influenced the study outcomes. ${ }^{33}$

Lastly, in our sample, scores on the HADS-A and HADS-D were relatively low (indicating 'no cases'). As shown in a recent overview by the Managing Epilepsy Well network, self-management may have an increased potential in patient who are diagnosed as having additional mental complaints such as depression. ${ }^{34}$

Strengths of our study were the low drop-out rate and the limited amount of missing data. Data were analysed using a mixed model, allowing us to impute missing data. Furthermore, the study population was evenly spread over all age groups.

One of the limitations of our study was the fact that the smartphone application 'Eppy' was not maintained and updated and eventually removed from the App Store (Apple Inc. USA and Google Play store, USA) early in 2015. Therefore, only a small group of the participants were able to use the application without experiencing problems with the system. Secondly, in order not to lose interested PWE during certain periods (e.g. holidays) when recruitment rate was low, we started block randomization at a minimum of six PWE; this was a deviation from the protocol.

Finally, our sample size was based on a standard deviation of 7 points on the ESES. In this study, we found a standard deviation of $>30$ points. The decrease in study power might have resulted in failure to detect statistically significant findings.

We acknowledge that further research is necessary to establish the overall effectiveness of the $\mathrm{MCl}$. This should take its place alongside process evaluation and economic evaluation of the intervention, both of which showed more favourable outcomes. ${ }^{21,22}$ The neurologists 
who recruited participants believed the intervention to be fruitful for both PWE and neurologist: the former showing more confidence and being prepared, and the latter experiencing a more effective consultation time; this was, however, not measured in this study.

A recently developed instrument for measuring the effectiveness of self-management interventions in epilepsy, the Adult Epilepsy Self-Management Measurement Instrument (AESMMI), may prove to be a great addition to future research. The AESMMI provides an overall effectiveness measure for self-management interventions in epilepsy ${ }^{35}$ and is a synthesis of 11 instruments, including the ESES and the QOLIE-31P.

We did not measure mastery, a concept that one can control options/events in life. ${ }^{36}$ Mastery experiences are the most effective way of becoming more self-efficacious ${ }^{14}$ and are associated with quality of life and emotional functioning. ${ }^{32}$ Hence, this would seem to be an important concept to measure in future research.

In conclusion, in spite of the lack of evidence that the $\mathrm{MCl}$ is effective looking at selfefficacy, we consider the $\mathrm{MCl}$ to be a promising intervention, the reduction of side-effects and on improving quality of life on subscales. Further research is needed, but we believe it should be carried out alongside implementation in order to reach a larger group of PWE. We recommend focusing on mastery and the domains of self-management as measured with the AESMMI, as they include most of the well-established outcome measures in epilepsy research (i.e. quality of life, adherence, side-effects, emotional functioning). 


\section{REFERENCES}

1. Taylor RS, Sander JW, Taylor RJ, Baker GA. Predictors of health-related quality of life and costs in adults with epilepsy: a systematic review. Epilepsia 2011; 52: 2168-80.

2. Baker GA. The psychosocial burden of epilepsy. Epilepsia 2002; 43 Suppl 6: 26-30.

3. Ryan P, Sawin KJ. The Individual and Family Self-Management Theory: background and perspectives on context, process, and outcomes. Nurs Outlook 2009; 57: 217-225 e6.

4. Bodenheimer T, Lorig K, Holman H, Grumbach K. Patient self-management of chronic disease in primary care. JAMA 2002; 288: 2469-75.

5. Faber M, Harmsen M, van der Burg S, van der Weijden T. Gezamenlijke besluitvorming \& Zelfmanagement: Een literatuuronderzoek naar de effectiviteit en naar voorwaarden voor succes. In. Nijmegen: Scientific Institute for Quality of Healthcare (IQ healthcare); 2013.

6. Jones RM, Butler JA, Thomas VA, Peveler RC, Prevett M. Adherence to treatment in patients with epilepsy: Associations with seizure control and illness beliefs. Seizure 2006; 15: 504-508.

7. Haynes RB, Ackloo E, Sahota N, McDonald HP, Yao X. Interventions for enhancing medication adherence. Cochrane Database Syst Rev 2008: CD000011.

8. Samsonsen C, Reimers A, Bråthen G, Helde G, Brodtkorb E. Nonadherence to treatment causing acute hospitalizations in people with epilepsy: An observational, prospective study. Epilepsia 2014; 55: e125e128.

9. Demonceau J, Ruppar T, Kristanto P, Hughes DA, Fargher E, Kardas P, et al. Identification and Assessment of Adherence-Enhancing Interventions in Studies Assessing Medication Adherence Through Electronically Compiled Drug Dosing Histories: A Systematic Literature Review and Meta-Analysis. Drugs 2013; 73: 545562.

10. Leenen LA, Wijnen BF, de Kinderen RJ, van Heugten CM, Evers SM, Majoie MH. Are people with epilepsy using eHealth-tools? Epilepsy Behav 2016; 64: 268-272.

11. Lorig KR, Ritter PL, Laurent DD, Plant K. Internet-based chronic disease self-management: a randomized trial. Med Care 2006; 44: 964-71.

12. England MJ, Liverman CT, Schultz AM, Strawbridge LM. Epilepsy across the spectrum: promoting health and understanding. A summary of the Institute of Medicine report. Epilepsy Behav 2012; 25: 266-76.

13. Amir M, Roziner I, Knoll A, Neufeld M. Self-Efficacy and social Support as Mediators in the Relation Between Disease Severity and Quality of Life in Patients with Epilepsy. Epilepsia 1998; 40: 216-224.

14. Benight CC, Bandura A. Social cognitive theory of posttraumatic recovery: the role of perceived self-efficacy. Behav Res Ther 2004; 42: 1129-48.

15. Pramuka M, Hendrickson R, Zinski A, Van Cott AC. A psychosocial self-management program for epilepsy: a randomized pilot study in adults. Epilepsy Behav 2007;11: 533-45.

16. Aspinwall LG, Taylor SE. A stitch in time: self-regulation and proactive coping. Psychol Bull 1997; 121: 41736.

17. de Silva D. Evidence: Helping people help themselves- a review of the evidence considering whether it is worthwhile to support self-management. In. Londen: Health Foundation; 2011.

18. Shegog R, Bamps YA, Patel A, Kakacek J, Escoffery C, Johnson EK, et al. Managing Epilepsy Well: Emerging e-Tools for epilepsy self-management. Epilepsy Behav 2013; 29: 133-40.

19. Ben-Menachem E, Biton V, Jatuzis D, Abou-Khalil B, Doty P, Rudd GD. Efficacy and safety of oral lacosamide as adjunctive therapy in adults with partial-onset seizures. Epilepsia 2007; 48: 1308-17.

20. Leenen LA, Wijnen BF, de Kinderen RJ, Majoie MH, van Heugten CM, Evers SM. (Cost)-effectiveness of a multi-component intervention for adults with epilepsy: study protocol of a Dutch randomized controlled trial (ZMILE study). BMC Neurol 2014; 14: 255.

21. Wijnen BFM, Leenen LAM, de Kinderen RJA, van Heugten CM, Majoie M, Evers S. An economic evaluation of a multicomponent self-management intervention for adults with epilepsy (ZMILE study). Epilepsia 2017; 58: 1398-1408. 
22. Leenen LAM, Wijnen BFM, van Haastregt JCM, de Kinderen RJA, Evers S, Majoie M, et al. Process evaluation of a multi-component self-management intervention for adults with epilepsy (ZMILE study). Epilepsy Behav 2017; 73: 64-70.

23. Thoolen B, de Ridder D, Bensing J, Gorter K, Rutten G. Beyond Good Intentions: the development and evaluation of a proactive self-management course for patients recently diagnosed with type 2 diabetes. Health Educ Res 2008; 23: 53-61.

24. Cramer JA, Van Hammee G, Group NS. Maintenance of improvement in health-related quality of life during long-term treatment with levetiracetam. Epilepsy Behav 2003; 4: 118-23.

25. Bell ML, Fairclough DL, Fiero MH, Butow PN. Handling missing items in the Hospital Anxiety and Depression Scale (HADS): a simulation study. BMC Res Notes 2016;9: 479.

26. Field A. Discovering Statistics Using SPSS: Sage Publications; 2009.

27. Bjelland I, Dahl AA, Haug TT, Neckelmann D. The validity of the Hospital Anxiety and Depression Scale. An updated literature review. J Psychosom Res 2002;52: 69-77.

28. Dilorio C, Escoffery C, McCarty F, Yeager KA, Henry TR, Koganti A, et al. Evaluation of WebEase: an epilepsy self-management Web site. Health Educ Res 2009; 24: 185-97.

29. Wang L, Zhang Z, McArdle JJ, Salthouse TA. Investigating Ceiling Effects in Longitudinal Data Analysis. Multivariate Behav Res 2009; 43: 476-496.

30. Tarquinio C, Kivits J, Minary L, Coste J, Alla F. Evaluating complex interventions: perspectives and issues for health behaviour change interventions. Psychol Health 2015; 30: 35-51.

31. Risdale L, Wojewodka G, Robinson E, Landau S, Noble A, Taylor S, et al. Characteristics associated with quality of life among people with drug-resistant epilepsy. J Neurol 2017; 264: 1174-1184.

32. Craig P, Dieppe P, Macintyre S, Michie S, Nazareth I, Petticrew M. Medical Research Council Guidance. Developing and evaluating complex interventions: the new Medical Research Council guidance. BMJ 2008; 337.

33. Ayling K, Brierley S, Johnson B, Heller S, Eiser C. How standard is standard care? Exploring control group outcomes in behaviour change interventions for young people with type 1 diabetes. Psychol Health 2015; 30: 85-103.

34. Sajatovic M, Jobst BC, Shegog R, Bamps YA, Begley CE, Fraser RT, et al. The Managing Epilepsy Well Network:: Advancing Epilepsy Self-Management. Am J Prev Med 2017; 52: S241-S245.

35. Escoffery C, Bamps Y, LaFrance WC, Jr., Stoll S, Shegog R, Buelow J, et al. Factor analyses of an Adult Epilepsy Self-Management Measurement Instrument (AESMMI). Epilepsy Behav 2015; 50: 184-9.

36. Pearlin LI, Schooler C. The structure of coping. J Health Soc Behav 1978; 19: 2-21.

37. Dilorio C, Yeager K. The Epilepsy Self-Efficacy Scale. In: Strickland O, Dilorio C, editors. Measurement of nursing outcomes: Self Care and Coping. 2nd ed. New York: Springer Publishing Company; 2003, p. 40-51.

38. Scholz U, Gutiérrez Doña B, Sud S, Schwarzer R. Is General Self-Efficacy a Universal Construct?1. European Journal of Psychological Assessment 2002;18: 242-251.

39. Menckeberg TT, Bouvy ML, Bracke M, Kaptein AA, Leufkens HG, Raaijmakers JA, et al. Beliefs about medicines predict refill adherence to inhaled corticosteroids. J Psychosom Res 2008; 64: 47-54.

40. O'Donoghue MF, Duncan JS, Sander JW. The National Hospital Seizure Severity Scale: a further development of the Chalfont Seizure Severity Scale. Epilepsia 1996; 37: 563-71.

41. Zigmond AS, Snaith RP. The hospital anxiety and depression scale. Acta Psychiatr Scand 1983; 67: 361-70.

42. Devinsky O, Vickrey BG, Cramer J, Perrine K, Hermann B, Meador K, et al. Development of the quality of life in epilepsy inventory. Epilepsia 1995; 36: 1089-104

43. Bode C, Thoolen B, Ridder d. Het meten van proactieve copingvaardigheden. Psychologie \& gezondheid 2008; 36: 81-91.

44. Uijl SG, Uiterwaal CS, Aldenkamp AP, Carpay JA, Doelman JC, Keizer K, et al. A cross-sectional study of subjective complaints in patients with epilepsy who seem to be well-controlled with anti-epileptic drugs. Seizure 2006; 15: 242-8. 
CHAPTER

General Discussion

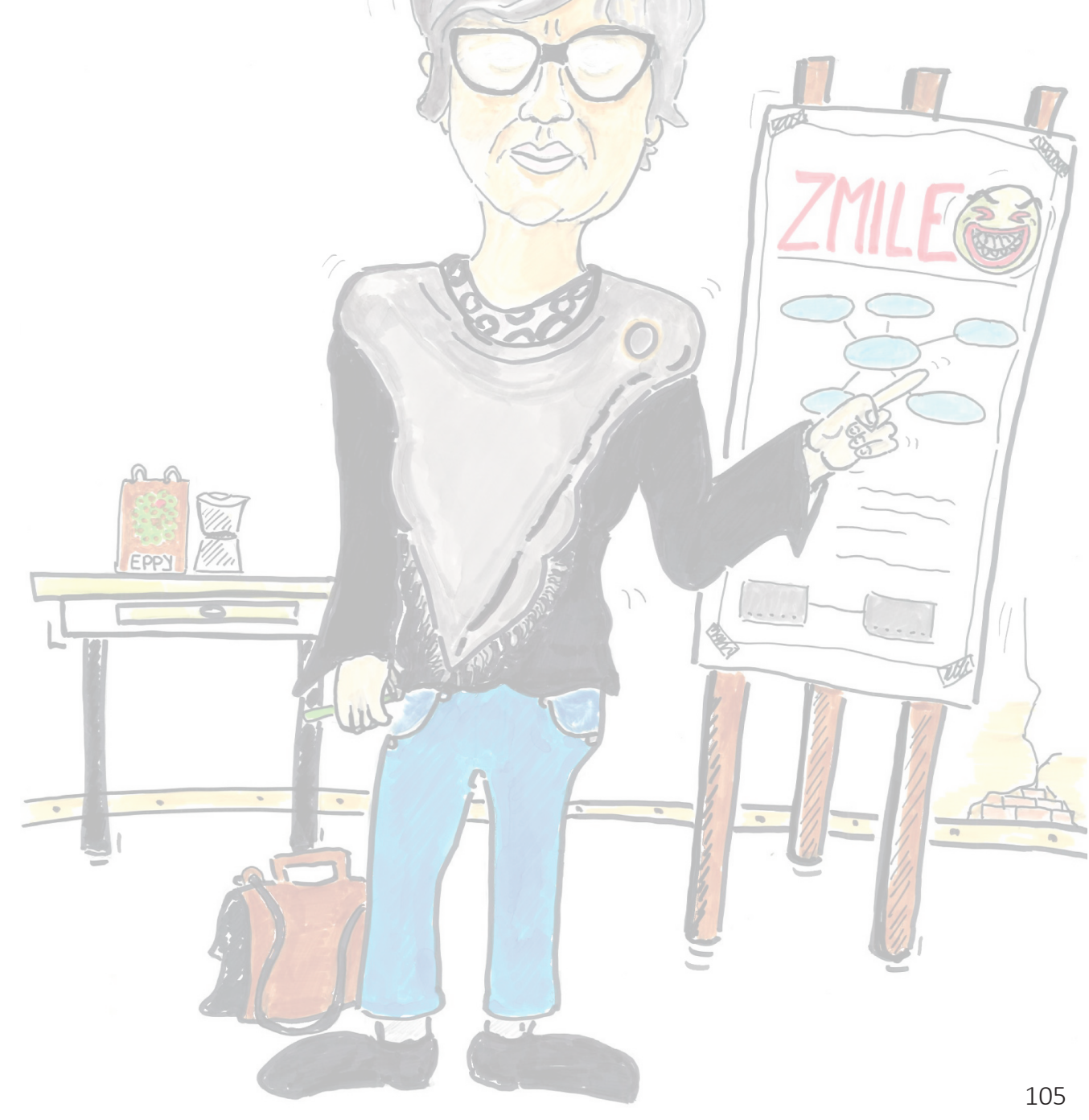


After a short reflection on the main aims of this $\mathrm{PhD}$ thesis and a description of the groupbased self-management intervention i.e. the Multi Component Intervention ( $\mathrm{MCl}$ ), this Chapter continues by discussing the methodological strengths and limitations of various studies presented in this thesis. Finally, the clinical implications and directions for further research are presented.

\section{AIMS OF THE THESIS}

This thesis evaluates the ZMILE-study, the main objective of which was to examine the feasibility, clinical, and cost-effectiveness of the $\mathrm{MCl}$ for persons with epilepsy (PWE) and their relatives, compared to care as usual. The $\mathrm{MCl}$ is aimed at 1) providing knowledge about self-monitoring and risk management, by using eHealth tools and 2) changing behaviour by emphasising proactive coping, concordance and goalsetting. In addition, we examined whether eHealth tools were applicable for epilepsy patients. The studies described in this thesis, therefore, aimed to answer the following main questions:

4. Are eHealth-tools usable tools to address self-management in persons with epilepsy?

5. Is a group-based, epilepsy-specific self-management intervention feasible?

6. Is a group-based, epilepsy-specific self-management intervention (cost)-effective?

- Is a group-based, epilepsy-specific self-management intervention compared to care as usual more effective in increasing self-efficacy and other patient reported outcomes?

- Is a group-based, epilepsy-specific self-management intervention preferable to care as usual from a societal perspective in terms of costs and effects?

The Multi-Component Self-Management Intervention for adults with epilepsy

'The ability to adapt and implement one's own control, in light of the physical, emotional and social challenges of life ${ }^{1}$, including self-management is a new definition of health. In order to self-manage, one needs to acquire knowledge and skills to become more selfefficacious, as shown in the model on which our $\mathrm{MCl}$ is based (Figure 7.1). Central to the $\mathrm{MCl}$, are the concepts of self-efficacy and pro-active coping. 


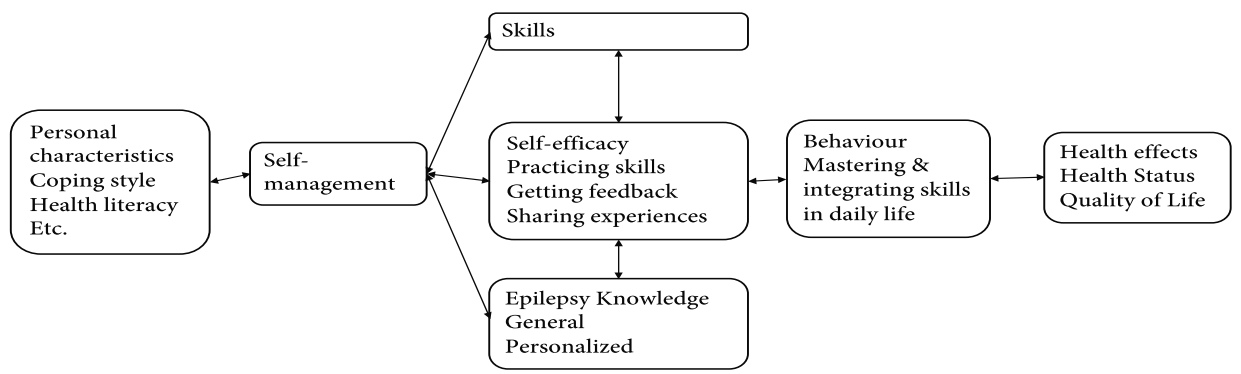

Figure 7.1: Theoretical model of the multi-component self-management intervention (adapted from Lemmens et al, 2008)

\section{Self-efficacy and pro-active coping}

Self-efficacy, defined as, 'the belief in one's capabilities to organize and execute the courses of action required to produce given attainments ${ }^{2}$ is often used in self-management interventions as a predictor of better health-related outcomes., ${ }^{3,4}$ Self-efficacy theory is based on the confidence a person has to accomplish actions or reach a desired goal. Self-efficacy is an important element in initiating and persisting with health-behaviour, and in determining the amount of time spent on sustaining the behaviour. ${ }^{5}$

Proactive coping is a coping strategy to prevent or modify a potential problem situation before it actual happens, while coping is defined as 'a person's cognitive and behavioural effort to reduce, minimize, master or tolerate the internal and external demands of the personenvironment transaction that is appraised as taxing or exceeding the person's resources'. ${ }^{6}$

People plan actions, while anticipating future threats, to reach their desired goal. Mastering these actions requires cognitive processes, motivation, pro-active coping capabilities, and a beneficial environment, which can be accomplished much easier by people with a high sense of self-efficacy. ${ }^{5}$

In the $\mathrm{MCl}$, we used the five-step framework as a tool for pro-active coping to enable PWE and their relatives to anticipate future barriers in their daily life, by preparing themselves to prevent or master these consequences and thus reach their desired goal. This five-step framework is based on Aspinwall and Taylors' five stages of proactive coping, namely: resource accumulation; recognition of potential stressors; initial appraisal; preliminary coping efforts; and elicitation and use of feedback concerning initial efforts. ${ }^{7}$

During the intervention, participants worked on their personal proactive action plan, leading to a personalized goal (behaviour). In order to stimulate self-management behaviour, goal-setting is used in relation to specific topics (e.g. self-esteem, sleep, adherence) in self-management interventions. ${ }^{8-10}$ The participants in our intervention chose a personal goal and learned proactive coping strategies to formulate and refine this personal goal to become achievable, during the sessions of the intervention. 


\section{MAIN RESULTS}

\section{Research question 1:}

Are eHealth-tools usable tools to address self-management in persons with epi-

The use and availability of eHealth tools was evaluated in Chapter 3, in which it was examined 1) how many PWE own hardware compared to the Dutch population in general; and 2) the advantages and disadvantages of eHealth as perceived by PWE - both users and non-users of eHealth-tools. This study demonstrated that, compared to the general population, there is a lack of access to a personal computer or smartphone among PWE. Self-monitoring activities are carried out more often by PWE in comparison with the general population, although not using eHealth tools. The perception of PWE is that eHealthtools are likely to be useful in supporting their self-management, but they are cautious with regard to their privacy, losing data, and the usability for all patients (equity). These results indicate that a more tailored approach is needed to stimulate the use of eHealthtools, for instance to improve self-management (Chapter $\mathbf{3}$ ).

In addition, though not solely focussed on eHealth, this is an important component in the overall $\mathrm{MCl}$ (Chapters 2 and 4 ). In the $\mathrm{MCl}$, the eHealth component consisted of selfmonitoring using a medication adherence monitoring system (MEMS) and a smartphone application designed for PWE. Participants were instructed to use eHealth as a tool to self-monitor, with the intention to provide them with knowledge on eHealth and eHealthtools, as well as the skills to use these tools in their self-management of epilepsy. eHealth was mentioned by the participants as an important (future) element and, therefore, an important 'learning to use' item within the intervention. This is also demonstrated by the fact that PWE frequently asked whether they were allowed to use the MEMS after the study period. (Chapter 4). Both studies highlight the importance and/or usefulness of eHealth in epilepsy.

In the literature, eHealth is widely recognized as an important addition to traditional health care. It provides an opportunity to improve access, reduce costs, and improve quality of health care. It also offers the prospect of improving quality of life in patients by empowerment to particpate in their own care, or to improve their self-management in general. ${ }^{11-13}$ To address the rapid development of eHealth, the Dutch government has incorporated the stimulation of eHealth in their objectives: eHealth, defined as the use of contemporary information and communication technologies, internet technology in particular. ${ }^{14}$ eHealth includes various electronic, wireless, and mobile devices; resources; and practices to support or improve health and healthcare. ${ }^{15}$ The availability and use of eHealth are mapped annually and presented in 'eHealth monitors', which also give an insight into incentives, obstacles, effects and developments over time. ${ }^{16}$ Both the eHealth monitor 2017 and our eHealth study, showed some similar outcomes. 
The first similarity is that about $50 \%$ of chronically ill patients or PWE self-monitor health values. Secondly, most of these health values were recorded on paper or in the device itself and not transmitted, or shared with health care professionals. ${ }^{16}$ This, in spite of evidence that communication or the transfer of information between health care provider and patients using eHealth is recognized as improving health outcomes in some chronic conditions (e.g. diabetes and heart failure). ${ }^{17}$ In epilepsy, the use of digital seizure calenders, incorporating information about seizure frequencies and/or effects of medication over time, have been shown to be helpful to patients and can also provide feedback to the users in the form of a graph. ${ }^{18,19}$

The third similarity is that almost one-third of the persons with a chronic condition did not want to measure health values according to the eHealth-monitor $2017^{16}$ or did not want to use or perceive advantage in using eHealth as our study showed.

This third outcome raises an important question, namely: 'Why does almost one-third of the intended population not want to self-measure or use eHealth?' This question is not really addressed by the eHealth monitor, although it is suggested that a proportion of the intended population do not need to measure. ${ }^{16}$

The question above fits in the recently added third-level digital divide, which addresses the question 'Why do some people benefit more from the internet than others? ${ }^{20,21}$

The early research on eHealth or the digital divide, defined as inequalities in access to, and use of Information and Communication Technologies (ICT), focussed on the inequality of owning a computer or not (first-level digital divide). Later on, one agreed that skills are also needed to use computers/the internet (second-level digital divide). ${ }^{20-23}$ Nowadays, attention must be paid to the third-level digital divide and the question it raises about why people who could or should benefit from web-based interventions do not benefit, in spite of the potential of these interventions to change health outcomes. ${ }^{24-}$ 26

Latulippe $(2017)^{23}$ showed that individual characteriscts play a role in the whole process of engaging people in the use of eHealth-tools. This process can best be described as firstly, for people to use eHealth, they must be motivated to seek support for healthrelated issues or to improve their health by a eHealth tool. Patients do not always know that - or how - self-management behaviour can influence health outcomes (clinical or patient-centred outcomes) or they think there is no need for self-management support. $^{16,27}$

Secondly, if people are motivated they, of course, need access to the digital technology. This no longer seem to be a big problem in Europe, since in 2017 an access level of $87 \%$ to the internet at home and $63 \%$ with a smartphone has been reached; for the Netherlands these figures are, respectively, $98 \%$ and $84 \% .{ }^{28}$

There are, however, still groups of people, especially those with a chronic condition, who have less access to the internet, as shown in both the eHealth monitor and our eHealth study. 
Having access is it in itself not enough. The third factor is that people need to have the confidence in their abilities to use this technology or in the technology itself to continue the process of seeking support for their health issues. ${ }^{29}$ Finally, having sufficient confidence in one's ability, is not enough to understand and comprehend the information offered by eHealth: 'health literacy'. In the Netherlands, about $50 \%$ of the population have sometime difficulty coping with health issues, ${ }^{30}$ and Hardiker et al. ${ }^{31}$ demonstrated that one should be especially aware of those who are under-served by eHealth services due to age, ethnicity, educational attainment and socioeconomic status.

In conclusion, although it has been shown that exposure to eHealth services appears to improve both the perceptions of non-users and frequency of use $\mathrm{e}^{31}$, little research has focussed on this perception of (possible) users of eHealth, nad perception of (dis)advantage of eHealth plays an important role for intended users, in deciding whether or not to use eHealth, as shown in our study.(see Chapter $\mathbf{3}$ )

To realise the potential of eHealth in self-care for the group of patients who need (self-management) support, to increase awareness of and skills to use eHealth tools, a more tailored approach is necessary, as recommended in the existing literature and in our study. ${ }^{16,32}$ Special attention should be placed on the fact that eHealth is not a replacement for face to face contact with their health care provider, but an addition to it. $^{8,27,32}$

We concluded, based on the results of the eHealth study, that a tailored approach was the best way to promote self-care or self-management in PWE, since some concepts were seen both as an advantage and as a disadvantages, depending on the perception of the respondent.(see Chapter 3 ). In addition, this tailored approach should include known characteristics, such as epilepsy severity and the presence of mental disorders (e.g. depression) which affect the use of online resources for health management. ${ }^{33}$ Also, some respondents in our study stated that by using eHealth, 'the health care professional always knows if there is something wrong with me'. A tailored approach could provide the opportunity to manage these expectations of eHealth users.

\section{Research question 2:}

Is a group-based, epilepsy-specific, self-management intervention feasible?

Although feasibility can refer to many aspects of an intervention, such as reach of desired target population or maximum treatment capacity, the study described in Chapter 4 (process evaluation) focussed on performance as described in the protocol (dose delivered), attendance and adherence (reach, dose received exposure and recruitment), and the patients', relatives' and care professionals' opinion about the $\mathrm{MCl}$ (dose received satisfaction). This study showed that the $\mathrm{MCl}$ reached the target audience, and overall that participants (i.e. PWE and their relatives) and facilitators (i.e. nurse practitioners) were satisfied with the intervention. Attendance rate and engagement of the participants was high; also, adherence of the facilitators to the protocol was optimal. (Chapter 4). 
It has been argued that, to evaluate an intervention to establish its feasibility, RCTs should include qualitative research. ${ }^{34,35}$ Qualitative research can be done using a number of frameworks specifically designed with a strong focus on the evaluation of the feasibility of a study, such as the framework of Saunders ${ }^{36}$ or of the Medical Research Centre $(\mathrm{MRC}){ }^{35}$ Such an evaluation can be done before, during or after (i.e. dissemination of) the RCT; and reporting of the result can be done simultaneously or separately from the results of the RCT. ${ }^{34}$ In the ZMILE-study we performed a process evaluation, based on the framework of Saunders, alongside the (cost-) effectiveness study of the $\mathrm{MCl}$. By combining quantitative and qualitative data, collected during and after the trial, we were able to assess information useful for interpreting the results of (cost-) effectiveness study and later in the dissemination process. It is, however, still not common to use qualitative research (i.e. process evaluations) in epilepsy care. ${ }^{37,38}$

To our knowledge, a feasibility assessment has only been in a few studies on epilepsy self-management interventions done, namely in WebEase ${ }^{39}$, PACES $^{8}$ and the SMILE (UK) study. ${ }^{40}$

In the WebEASE study, the feasibility, usability and accessibility of their internet-based programme was assessed, alongside a preliminary assessment of behaviour and attitude changes of the participants. The conclusion from both qualitative (group interviews) and quantitative data (questionnaires) was that the participant valued the programme, and found it to be a relevant, acceptable and easy to use programme. Furthermore, the results of this study indicated that the theoretical components, on which the internet-based programme was built, were successfully integrated into the programme. ${ }^{39}$ In the PACES study, no clear description was given on how feasibility was assessed alongside the effectiveness study. The result section noted, however, that the value of the programme was assessed in both groups (treatment and control) and that participants valued both the content and how the elements were delivered. Recommendations for improving PACES were made, such as longer follow-ups, more booster sessions, the use of web-based or telephone follow-ups and changing the ways goal-setting was delivered. ${ }^{8}$ In the recently conducted SMILE study, two process evaluations were conducted both of which were separately published. The first focussed on assessing implementation fidelity, with a specially designed fidelity measurement instrument, alongside the (cost) effectiveness study. This instrument focusses on adherence and competence of the facilitators as constructs of fidelity. The conclusion of this study was that their specially designed fidelity instrument was easy to use and showed that the SMILE intervention was delivered according to protocol. ${ }^{41}$

The second qualitative evaluation was performed after the trial. This evaluation was done by in-depth interviews with former participants of the RCT, to assess the value of the intervention from a user perspective. Although the intervention was valued by the participants, there were some important issues, such as lack of sufficient understanding of English and memory problems, which could affect the feasibility of the intervention. ${ }^{42}$ 
Besides the variability on effectiveness and the heterogeneity in interventions, it can also be concluded that the number of qualitative research studies focussing on feasibility is relatively small, and that there is a large variety in the way feasibility or process evaluations are being conducted. This makes a comparison between studies almost impossible. Also, the results of the feasibility studies are often aimed at improving the intervention instead of making statements on its effectiveness. For example, from the above studies it becomes clear that possible components which contribute to the feasibility of selfmanagement interventions in epilepsy might be: a competent, adherent facilitator ${ }^{41}$, sessions spread over time with booster sessions eventually carried out by telephone or webbased $^{8}$, and taking into consideration problems which can arise for participants of the interventions, such as functional illiteracy and memory problems. ${ }^{42}$ In line with our process evaluations, Wojewodka et al. ${ }^{41}$ also determined whether the intervention was delivered as planned, albeit in a different way (focussing mainly on fidelity).

In addition, a review ${ }^{27}$ on patients' perspective on limiting factors influencing the decision to enrol in a self-management intervention were: limited knowledge and understanding about how to self-manage, the belief that one would not benefit from a selfmanagement intervention, and lack of transportation. Enabling factors were also mentioned, namely: lack of social support at home, (thus hoping to get support from peers), suitable time and duration of the intervention, confidence in the health care centre delivering the intervention, and the belief it is one's duty to participate in order to establish better health care. ${ }^{27}$ This is in line with our findings in the process evaluation of the ZMILEstudy, in which it was suggested that feasibility could be improved by, among others, planning the booster session at a later time and managing expectations about the $\mathrm{MCl}$.

In conclusion, group-based, epilepsy-specific self-management interventions are feasible. However, there is a need for an increased awareness of currently available standardized frameworks (such as Saunders et al. 2009) ${ }^{36}$, in order to develop comprehensive and comparable feasibility studies. In addition, there is a need to incorporate qualitative data collection on a regular basis when conducting clinical trials, to reveal user or patient perspectives and to highlight possible problems in implementation from the point of view of the intended user. Moreover, results of process evaluations are often poorly reported as they are often included in (cost-)effectiveness papers instead of being published as a standalone paper.

\section{Research question 3:}

Is a group-based, epilepsy-specific, self-management intervention (cost)-effective?

To answer this question, we performed a randomized controlled trial (RCT) in adult patients with epilepsy (see Chapter $\mathbf{2}$ for the study protocol and Chapters $\mathbf{5}$ and $\mathbf{6}$ for the results of the clinical and cost effects resp.). Participants were randomized to either the intervention or to the care as usual (CAU) group. Data were collected at baseline and after three and six months in the comparative part of the trial. The intervention group 
had an extended follow-up after nine and 12 months. Data was collected using questionnaires and by a Medication Event Monitoring System (MEMS), the latter providing data about dates and times a pill box was opened.

Although we found improvements in self-efficacy (primary outcome) between the intervention and CAU group, this difference was not significant. In the intervention group compared to CAU, we found significant improvement in the secondary outcomes i.e. quality of life in epilepsy (on mood, daily life and distress); a decrease in anti-epileptic side-effects; and an improvement in adherence rate (not significant). (Chapter 6)

For the economic evaluation part of the study, we assessed: adherence; self-efficacy (ESES), quality adjusted life years (QALYs); health care costs; production losses; and patient \& family costs. Data were collected at baseline and during the 12-month study period. Incremental cost-effectiveness ratios (ICERs) (i.e. cost per increased adherence, selfefficacy or QALY), and cost-effectiveness acceptability curves were calculated and presented. Although not statistically significant, cost-effectiveness appeared to be promising based on the mean net benefit in our sample. When looking at the QALYs (Dutch tariff), an ICUR of $€ 8,272$ and $€ 15,144$ per QALY gain was found at 6 and 12 months follow-up, respectively; this is below the threshold of $€ 50,000$ Euro per QALY. Considering this threshold, the probability that the $\mathrm{MCl}$ was cost-effective was estimated to be $74 \%$ at 6 months and $76 \%$ at 12 months of follow-up (Chapter 5).

It is difficult to compare our group-based, epilepsy-specific, self-management intervention with other epilepsy self-management interventions ${ }^{8,39,40}$ due to the large variation between studies. Reviews of epilepsy self-management interventions showed that the interventions differ with regard to their focus; intended goal(s); facilitators (i.e. nurse practitioners, psychologists, neurologists); method of delivering (e.g. leaflet; internet, telephone, text messages etc.) and the number of contacts within the intervention. In addition, interventions often consist of several components which makes it difficult to establish which contributes most to the demonstrated effects. ${ }^{37,38,43}$ Some suggestions have been made to increase effectiveness of the interventions, such as using a nurse specialist, focussing on self-management education ${ }^{37}$, using booster sessions, and including a psychological component ${ }^{38}$; these were the elements we included in our intervention.

A few studies investigated the cost-effectiveness of self-management in epilepsy. In a review of Wijnen et al. (2017), only three studies were identified. ${ }^{44}$ Beretta et al. $(2014)^{45}$ compared a standard educational plan with usual care only (i.e., medical management). However, the authors did not include incremental analyses. Moreover, Plumpton et al. (2015) ${ }^{46}$ conducted an economic evaluation of a behaviour-modifying intervention to enhance AED adherence. This programme resulted in fewer costs and more effective, and therefore dominated care as usual. ${ }^{46}$ Lastly, in an underpowered study, Noble et al. (2014) determined the cost-effectiveness of a self-management programme for adults with epilepsy attending the emergency department (ED). This intervention resulted in slightly lower costs and fewer admission days after ED visits, but no effect in total ED visits or on psychosocial outcomes. ${ }^{47}$ 
Based on these studies, especially due to the large variability in economic evaluations in epilepsy (in terms of content of the programmes but also in their methodology), it is difficult to make strong comparisons and to draw firm conclusions regarding the costeffectiveness of self-management in epilepsy. ${ }^{44}$

Some of the previously conducted studies do show improvements in self-efficacy by those attending self-management programmes in epilepsy ${ }^{9,10}$ or other chronic conditions. ${ }^{48,49}$ There are, however, also reports of no improvements in self-efficacy ${ }^{50,51}$ which is in line with our study. Although, we found improvement on secondary outcomes and the cost-effectiveness was below the threshold, the limited improvement in self-efficacy, can be explained by several reasons. Of course, it could simply mean that this self-management intervention is no more than having 'limited' effect in PWE. However, absence of evidence does not mean evidence of absence. And in this case, there are alternative explanations, which are discussed briefly in Chapters 5 and 6. Given the positive feedback from neurologists, PWE, and caregivers during the process evaluation, and given the positive trends in the secondary outcome measures in the trial, it is important to reflect on these alternative explanations. A first explanation could be that we used an outcome measure which is not sensitive to a relevant change. Most of the outcome measurements in our ZMILE-study are comparable to measurements in most epilepsy self-management intervention studies (e.g. seizure frequency, self-efficacy, quality of life, coping, etc.). Systematic reviews of these studies reported (significant) improvement in at least one measure in the intervention group compared to the control group. ${ }^{37,38,43}$ However, these significant results were not necessarily always the primary outcome measure of the reported studies, and often did not sustain when measurements were repeated after three or six months follow-up. ${ }^{38}$ The systematic reviews also reported a large variation in follow-up, varying from one month to two years. ${ }^{37,38,43}$

The most frequently used outcome measure (primary or secondary) was quality of life, but this was not used in all epilepsy-related, self-management interventions and some studies that showed a favourable effect, used their own constructed outcome measures. ${ }^{52,53}$ As mentioned earlier in this thesis, some subdomains of quality of life also improved significantly compared to the care as usual group in our sample (see Chapter 5).

However, a difference with the above-mentioned studies is that we did not measure (self-management) behaviour or behavioural change. Behavioural change is usually measured by patient-reported questionnaires, which often address only the intention to change health behaviour. Actual change is more difficult to measure. In the ZMILE-study, we assumed that behaviour would change as self-efficacy increases, which would be the expected mechanism of action following the theory of planned behaviour. Following this theory, self-efficacy is perceived as at least one of the components that predicts intention to change. ${ }^{54}$ Secondly, since we applied a patient-centred approach for proactive goalsetting, in which every participant could choose a personal goal, we believed this was difficult to measure. Especially since some of these goals seem, at first glance, not to be 
associated with specific self-management behaviour, but were rather tasks or problems participants faced in everyday life. Participants were taught the steps of the framework of planned action, in order to obtain knowledge and skills to address the problem or task they perceived, and to discuss their findings or ideas with group members. Changing behaviour could be perceived as a learning process, which can be looked at in different ways for instance, problem-based learning (PBL). PBL is a way of addressing problems not by offering a solution, but by acquiring knowledge, collaborating in a group, communicating about problem and solution and forming self-directed habits (i.e. behaviour). This PBL has been shown to be effective in long-term retention of knowledge and skills, ${ }^{55}$ and in chronic disease management, although the latter effect was measured in clinical outcomes such as glucose levels. The effect on behaviour (i.e. life style changes) was established by self-reported questionnaires and not by measuring the actual behavioural change. ${ }^{56}$

In spite of expecting that self-efficacy could capture the intended change in behaviour, we perhaps should have measured behaviour itself. The choice to measure the behavioural change in particular, and not a derivative thereof, is further substantiated by the fact that both the participants and the caregivers report positive behavioural changes in the process evaluation. For instance, neurologists reported that the participants prepared their visit more thoroughly, so that these consultations were fare more efficient, and participants stated they were not afraid to take part in certain activities alone, such as shopping or going to work by public transport. Such outcomes were not considered in our outcome measurements.

A third reason for the lack of significant positive effect on the primary outcome might be that there is no good outcome measure to evaluate self-efficacy as a self-management skill. None of the currently available studies used an instrument specifically developed to examine the effects of (multi-component) self-management interventions. This, in turn, may have led to a lack of sensitivity to detect relevant changes in a participants' health status. The Adult Epilepsy Self- Management Measurement Instrument (AESMMI) could be such an instrument. It assesses effectiveness of self-management interventions, using a synthesis of 11 other instruments including epilepsy-related self-efficacy and quality of life. ${ }^{57}$

Various reviews $s^{37,38,43}$ showed that the effectiveness or the lack of effectiveness of a self-management intervention can be partially explained by the choice of the outcome measures. For example, in effective self-management interventions for diseases such as asthma, diabetes or hypertension, clinical outcomes are usually applied, such as glucose levels or blood pressure.

Clinical outcomes can be assessed more easily and accurately, in contrast with patient-reported outcomes, which are subject to individual variability, and therefore more prone to bias. Patient-reported outcomes, especially those that require a high degree of cognitive appraisal, are subject to more individual variability and interpretation differences. ${ }^{58}$ Schwartz $(2004)^{59}$ proposed a model with three types of outcomes 
measurements (i.e. performance-based, perception-based and evaluation based). The most vulnerable outcomes in this model to response shift bias are evaluation-based measurement, such as anxiety, depression, pain, and also self-efficacy. Evaluation-based measurements require a high degree of cognitive appraisal to 1) interpretate the question, 2) retrieve relevant information, 3) process the relevant information, and 4) formulate the response to the question. The result of this appraisal process can differ both between persons as within persons as a result of learning processes during the intervention or the occurrence of significant events between follow-up visits. ${ }^{58,59}$

It is, therefore, possible that using an evaluation-based measurement as self-efficacy in the ZMILE-study may be appraised differently by participants before or after attending the intervention, due to reconceptualization because of social comparison with other group members. There is also the possibility that because participants meet others who perhaps seem more confident, they will change their opinion about the values of the scale. ${ }^{58}$

The fourth possible explanation is that participants were selected based on their willingness to participate in the ZMILE-study, not on their need for self-management support. Therefore, it is possible that the study group, which had a high, self-efficacy score before the start of the intervention, were already good self-managers, and not individuals with low health literacy, who needed more support. ${ }^{60}$

In conclusion, the challenge for society as a whole is to provide optimal care within a specific budget. This is especially challenging because of an aging population, accompanied by an increasing number of chronic diseases. One way of identifying optimal care is to conduct economic evaluations in which new interventions are compared to care as usual to determine the cost-effectiveness. National regulatory bodies around the world are becoming increasingly aware of this matter and are requiring economic evidence for the reimbursement assessment of new interventions. ${ }^{62,63}$ Following the example of the $\mathrm{NICE}^{64}$ and $\mathrm{NHS}^{65}$ guidelines, clinical guidelines developers aim at including economic evaluations as a consideration when formulating guideline recommendations. For example, since 2016, economic evaluations have been included in the updated clinical practice guidelines for epilepsy in the Netherlands. ${ }^{62}$ In spite of this growing need for economic evaluations, with the exception of the study described in Chapter 5 (the ZMILE economic evaluations), no other economic evaluations in the field of self-management in epilepsy have been conducted. ${ }^{44}$ In addition, the review by Wijnen et al. (2017) ${ }^{44}$ highlighted the lack of comparability between studies, making it hard to draw firm conclusions.

Nonetheless, the ZMILE-study demonstrated that a multi-component, self-management intervention has the potential to be a cost-effective addition to current care given the demonstrated improvements in some clinical outcomes and positive cost-effectiveness estimates. 


\section{METHODOLOGICAL STRENGTH AND CONSIDERATIONS}

The ZMILE-study is the first study to evaluate the cost-effectiveness of an MCl for PWE in the Netherlands. Such a study is important, because of the high burden of epilepsy in daily life which compromises the quality of life of PWE and their relatives and which has a large impact on society in terms of resource use and productivity losses. Our study has, therefore, been set out to evaluate the impact of the $\mathrm{MCl}$ in PWE and to evaluate whether the $\mathrm{MCl}$ is also economically sustainable.

The strength of the group-based $\mathrm{MCl}$ is that it has been developed according to MRC guidelines, a framework to develop and evaluate complex evaluation. ${ }^{35}$ According to this framework, developing and evaluating complex interventions goes through four interrelated phases: 1) development of the intervention based on the best available evidence and appropriate theory, 2) feasibility piloting, 3) assessing (cost-) effectiveness and complement this with a process evaluation, and 4) implementation. ${ }^{35}$

We developed the intervention based on a systematic review of self-management strategies for adults with epilepsy ${ }^{66}$, evidence-based interventions for chronic disease self-management programmes (CDSMP) ${ }^{67}$, an evidence-based intervention for diabetes $^{48}$, the pro-active coping theory ${ }^{7}$, the theory of planned behaviour ${ }^{54}$, and the Social Cognitive theory. ${ }^{2}$ Further fine tuning of the intervention took place based on the experience with the Plan Ahead intervention. ${ }^{68}$ The Plan Ahead intervention for stroke patients was used as the practical framework for the intervention and was discussed with our advisory board. To oversee all underlying assumptions, theories and evidence-based practises, the findings were put in a model (Figure 7.1). A pilot was performed to test the content of the intervention. During the pilot, we introduced a fictitious patient with a relative to help explain the five-step approach of proactive goal-setting. Sample size for the trial was calculated and recruitment rate was estimated.

Subsequently the (cost)-effectiveness of the $\mathrm{MCl}$ was evaluated by means of a randomized controlled trial (RCT), while the underlying processes were examined in a process evaluation, Also, a detailed treatment protocol was available for the facilitators who, prior to the intervention, received a training in motivational interviewing and how to conduct the intervention.

Another strength is that the intervention consists of multiple components. This meets the complexity of the challenges that people with epilepsy face when they want to develop self-management skills. Most self-management interventions focus on a single component, such as improving compliance or improving knowledge. According to Faber ${ }^{69}$, such an approach is not sufficient to increase people's self-management skills. A self-management intervention should, next to self-management education, include components such as peer support, goal-setting, shared decision-making and self-monitoring. ${ }^{69,70}$

As well as the various strengths of the studies, there were also limitations. First, although we intended to change (self-management) behaviour with our self-management 
intervention, we measured self-efficacy as a proxy for behaviour instead of measuring behaviour change. Secondly, although the group-based, self-management intervention consists of multiple components, which is a strength of the intervention, the fact that these multiple components can be interconnected can be a drawback, as they can act both independently and inter-dependently. ${ }^{71}$ This makes both favourable or unfavourable outcomes difficult to interpret and makes it difficult to establish which outcomes are effective and which are not. ${ }^{71}$

The third limitation is that our RCT was conducted within a naturalistic setting, in which the control group received care as usual. From a positive perspective, a natural setting increases the generalizability of the results and ensures that our sample more closely resembles daily practice. Conversely, this implies that our control group received care as usual, which may reflect a somewhat variable concept. Ideally the control group needs to receive care as uniformly as possible., On the whole this is difficult, because participants receive care from different health care providers. Furthermore, participants can have a variety of problems for which they can depend on their health care provider. Also, most of the participants of the control group received care from a specialized epilepsy centre, which delivers high quality care. A higher standard of care may have resulted in a lower effect size. ${ }^{72,73}$

In addition, all participants in the ZMILE-study received the Medication Event Monitoring System (MEMS) as part of the ZMILE-study. Although the CAU group did not receive any feedback on their use of the MEMS system, applying the system in both groups may have led to less contrast between groups, ultimately diminishing the effects of the $\mathrm{MCl}$.

\section{CONCLUDING REMARKS \& RECOMMENDATIONS}

The main part of this thesis focussed on the ZMILE-study. The $\mathrm{MCl}$ used in this study was thoroughly examined by means of a process evaluation, effectiveness evaluation, and an economic evaluation. Applying various form of evaluations shed a broad light on the effects and implications of the $\mathrm{MCl}$, including patients', relatives' and clinicians' perspectives. Results showed that the $\mathrm{MCl}$ was feasible and satisfied patients' needs, resulting in positive effects in some domains, for example reduction in side-effects and subdomains in quality of life. Importantly, results of the economic evaluations indicated promising net benefits, suggesting the $\mathrm{MCl}$ may be a cost-effective alternative given current societal willingness-to-pay thresholds. Using recommendations provided by patients, relatives, and clinicians, the $\mathrm{MCl}$ can be improved to increase and sustain effects over a longer time horizon.

The results described in this thesis have implications for clinical practice. Hence, based on the work presented here, the following recommendation can be made: 
In general:

To improve the effectiveness of a self-management intervention, awareness of the need for self-management and how it can influence health outcomes is required as patients and healthcare providers are not always aware of the importance of self-management. In the short term this could be improved by a preparatory intervention, such as motivational interviewing, but ideally, self-management should be part of the education of health care providers.

In developing interventions in the future, it is recommended to:

- Use a tailored approach when developing eHealth applications for PWE.

- Identify subgroups of patients for whom the $\mathrm{MCl}$ is particularly (cost-)effective.

- Improve an intervention based on the outcomes of the process evaluation, in order to better fit patients' needs and capabilities.

- Include booster sessions, which are known to sustain effect in the long-term.

For future studies evaluating interventions, it is recommended to:

- Standardly use the current available frameworks, for developing, evaluating and implementing complex interventions. ${ }^{35,74,75}$

- Identify sensitive and valid instruments to measure the effects of a $\mathrm{MCl}$, for instance, the recently developed Adult Epilepsy Self-Management Measurement Instrument (AESMMI).

- Increase emphasis on measurement of behavioural changes or/and mastery instead of measurement of self-efficacy alone.

- Always include an economic and a process evaluation when conducting a randomizedcontrolled trial.

- Publish the results of the effectiveness, the process evaluation, and the economic evaluation separately, in case these studies are combined to enhance transparency. 


\section{REFERENCES}

1. Huber M, Knottnerus JA, Green L, Horst Hvd, Jadad AR, Kromhout D, et al. How should we define health? BMJ 2011; 343.

2. Bandura A. Self-Efficacy: The Exercise of Control. 6th ed. New York: W.H. Freeman and Company; 2003.

3. Lorig KR, Holman H. Self-management education: history, definition, outcomes, and mechanisms. Ann Behav Med 2003; 26: 1-7.

4. Dilorio C, Shafer PO, Letz R, Henry TR, Schomer DL, Yeager K, et al. Project EASE: a study to test a psychosocial model of epilepsy medication management. Epilepsy Behav 2004; 5: 926-936.

5. Bandura A. Perceived Self-Efficacy in Cognitive Development and Functioning. Educational Psychologist 1993; 28: 117-148.

6. Folkman S, Lazarus RS, Gruen RJ, DeLongis A. Appraisal, coping, health status, and psychological symptoms. J Pers Soc Psychol 1986; 50: 571-9.

7. Aspinwall LG, Taylor SE. A stitch in time: self-regulation and proactive coping. Psychol Bull 1997; 121: 41736.

8. Fraser RT, Johnson EK, Lashley S, Barber J, Chaytor N, Miller JW, et al. PACES in epilepsy: Results of a selfmanagement randomized controlled trial. Epilepsia 2015; 56: 1264-74.

9. Dilorio C, Bamps Y, Walker ER, Escoffery C. Results of a research study evaluating WebEase, an online epilepsy self-management program. Epilepsy Behav 2011; 22: 469-74.

10. Pramuka M, Hendrickson R, Zinski A, Van Cott AC. A psychosocial self-management program for epilepsy: a randomized pilot study in adults. Epilepsy Behav 2007; 11: 533-45.

11. Department of Health UK. 2010-2015 Government policy: long term health conditions. In: https://www.gov.uk/government/publications/2010-to-2015-government-policy-long-term-healthconditions

12. IOM (Institute of Medicine). Epilepsy across the spectrum: promoting health and understanding. In. Washington DC: National Academies Press; 2012. p. 148-56.

13. Schippers E. Kamerbrief over E-Health.In: https://www.rijksoverheid.nl/onderwerpen/ehealth/ documenten/kamerstukken/2012/06/07/kamerbrief-over-e-health; 2012.

14. Krijgsman J, Klein Woltering G. Whitepaper Ordening in de wereld van eHealth. In: Nictiz; 2012.

15. Shegog R, Bamps YA, Patel A, Kakacek J, Escoffery C, Johnson EK, et al. Managing Epilepsy Well: Emerging e-Tools for epilepsy self-management. Epilepsy Behav 2013; 29: 133-40.

16. Wouters M, Swinkels I, Sinnige J, de Jong J, Brabers A, van Lttow B, et al. Kies bewust voor eHealth - eHealth monitor 2017. In: Nederlands instituut voor onderzoek van de gezondheidszorg (NIVEL) \& NICTIZ; 2017; In:https://www.nictiz.nl/SiteCollection Documents/ Rapporten/eHealth-monitor\%202017.pdf

17. Hanlon P, Daines L, Campbell C, McKinstry B, Weller D, Pinnock H. Telehealth Interventions to Support Self-Management of Long-Term Conditions: A Systematic Metareview of Diabetes, Heart Failure, Asthma, Chronic Obstructive Pulmonary Disease, and Cancer. Journal of Medical Internet Research 2017; 19: e172.

18. Fisher RS. Tracking epilepsy with an electronic diary. Acta Paediatr 2010; 99: 516-8.

19. Le S, Shafer PO, Bartfeld E, Fisher RS. An online diary for tracking epilepsy. Epilepsy Behav 2011; 22: 7059.

20. van Deursen AJAM, Helsper EJ. The Third-Level Digital Divide: Who Benefits Most from Being Online? In: Communication and Information Technologies Annual; 2015, p. 29-52.

21. Scheerder A, van Deursen A, van Dijk J. Determinants of Internet skills, uses and outcomes. A systematic review of the second- and third-level digital divide. Telematics and Informatics 2017; 34: 1607-1624.

22. Neter E, Brainin E. eHealth literacy: extending the digital divide to the realm of health information. J Med Internet Res 2012;14: e19.

23. Latulippe K, Hamel C, Giroux D. Social Health Inequalities and eHealth: A Literature Review With Qualitative Synthesis of Theoretical and Empirical Studies. J Med Internet Res 2017; 19: e136.

24. Schermer M. Telecare and self-management: opportunity to change the paradigm? Journal of Medical Ethics 2009; 35: 688. 
25. Murray E. Web-based interventions for behavior change and self-management: potential, pitfalls, and progress. Med 20 2012; 1: e3.

26. Shegog R, Begley CE. Clinic-Based Mobile Health Decision Support to Enhance Adult Epilepsy SelfManagement: An Intervention Mapping Approach. Front Public Health 2017; 5: 256.

27. Paige S, Stellefson M, Singh B. Patient perspectives on factors associated with enrollment and retention in chronic disease self-management programs: a systematic review. Patient Intelligence 2016.

28. Eurostat. In: http://ec.europa.eu/eurostat/tgm/table.do?tab=table\&init=1\&language=en\&pcode= tin00134\& plugin=1.; 2018.

29. McAuley A. Digital health interventions: widening access or widening inequalities? Public Health 2014; 128: $1118-20$.

30. Weten is nog geen doen - een realistisch perspectief of redzaamheid. In:Wetenschappelijke raad voor het regeringsbeleid; https://www.wrr.nl/publicaties/rapporten/2017/04/24/ weten-is-nog-geen-doen; 2017.

31. Hardiker NR, Grant MJ. Factors that influence public engagement with eHealth: A literature review. Int J Med Inform 2011;80: 1-12.

32. Huygens MW, Vermeulen J, Swinkels IC, Friele RD, van Schayck OC, de Witte LP. Expectations and needs of patients with a chronic disease toward self-management and eHealth for self-management purposes. BMC Health Serv Res 2016; 16: 232.

33. Crooks RE, Bell M, Patten SB, Wiebe S, Holroyd-Leduc J, Bulloch AG, et al. Mind the gap: Exploring information gaps for the development of an online resource hub for epilepsy and depression. Epilepsy Behav 2017; 70: 18-23.

34. Lewin S, Glenton C, Oxman AD. Use of qualitative methods alongside randomised controlled trials of complex healthcare interventions: methodological study. BMJ 2009; 339: b3496.

35. Craig P, Dieppe P, Macintyre S, Michie S, Nazareth I, Petticrew M. Developing and evaluating complex interventions: the new Medical Research Council guidance. Int J Nurs Stud 2013; 50: 587-92.

36. Saunders RP, Evans MH, Joshi P. Developing a process-evaluation plan for assessing health promotion program implementation: a how-to guide. Health Promot Pract 2005; 6: 134-47.

37. Bradley PM, Lindsay B, Fleeman N. Care delivery and self management strategies for adults with epilepsy. Cochrane Database Syst Rev 2016;2: CD006244.

38. Smith A, McKinlay A, Wojewodka G, Ridsdale L. A systematic review and narrative synthesis of group selfmanagement interventions for adults with epilepsy. BMC Neurol 2017; 17: 114.

39. Dilorio C, Escoffery C, McCarty F, Yeager KA, Henry TR, Koganti A, et al. Evaluation of WebEase: an epilepsy self-management Web site. Health Educ Res 2009; 24: 185-97.

40. Magill N, Ridsdale L, Goldstein LH, McCrone P, Morgan M, Noble AJ, et al. Self-management education for adults with poorly controlled epilepsy (SMILE (UK)): statistical, economic and qualitative analysis plan for a randomised controlled trial. Trials 2015; 16: 269.

41. Wojewodka G, Hurley S, Taylor SJC, Noble AJ, Ridsdale L, Goldstein LH. Implementation fidelity of a selfmanagement course for epilepsy: method and assessment. BMC Med Res Methodol 2017; 17: 100.

42. Ridsdale L, Philpott SJ, Krooupa AM, Morgan M. People with epilepsy obtain added value from education in groups: results of a qualitative study. Eur J Neurol 2017; 24: 609-616.

43. Michaelis R, Tang V, Wagner JL, Modi AC, LaFrance WC, Jr., Goldstein LH, et al. Psychological treatments for people with epilepsy. Cochrane Database Syst Rev 2017; 10: CD012081.

44. Wijnen BFM, van Mastrigt G, Evers S, Gershuni O, Lambrechts D, Majoie M, et al. A systematic review of economic evaluations of treatments for patients with epilepsy. Epilepsia 2017; 58: 706-726.

45. Beretta S, Beghi E, Messina P, Gerardi F, Pescini F, La Licata A, et al. Comprehensive educational plan for patients with epilepsy and comorbidity (EDU-COM): a pragmatic randomised trial. J Neurol Neurosurg Psychiatry 2014; 85: 889-94.

46. Plumpton CO, Brown I, Reuber M, Marson AG, Hughes DA. Economic evaluation of a behavior-modifying intervention to enhance antiepileptic drug adherence. Epilepsy Behav 2015; 45: 180-6.

47. Noble AJ, McCrone P, Seed PT, Goldstein LH, Ridsdale L. Clinical- and cost-effectiveness of a nurse led self-management intervention to reduce emergency visits by people with epilepsy. PLoS One 2014; 9 : e90789. 
48. Thoolen B, de Ridder D, Bensing J, Gorter K, Rutten G. Beyond Good Intentions: the development and evaluation of a proactive self-management course for patients recently diagnosed with type 2 diabetes. Health Educ Res 2008; 23: 53-61.

49. Zijlstra GA, van Haastregt JC, van Eijk JT, de Witte LP, Ambergen T, Kempen GI. Mediating effects of psychosocial factors on concerns about falling and daily activity in a multicomponent cognitive behavioral group intervention. Aging Ment Health 2011; 15: 68-77.

50. Smeulders ES, van Haastregt JC, Ambergen T, Uszko-Lencer NH, Janssen-Boyne JJ, Gorgels AP, et al. Nurseled self-management group programme for patients with congestive heart failure: randomized controlled trial. J Adv Nurs 2010; 66: 1487-99.

51. Rose MA, Arenson C, Harrod P, Salkey R, Santana A, Diamond J. Evaluation of the Chronic Disease SelfManagement Program with low-income, urban, African American older adults. J Community Health Nurs 2008; 25: 193-202.

52. May TW, Pfafflin M. The efficacy of an educational treatment program for patients with epilepsy (MOSES): results of a controlled, randomized study. Modular Service Package Epilepsy. Epilepsia 2002; 43: 539-49.

53. Ibinda F, Mbuba CK, Kariuki SM, Chengo E, Ngugi AK, Odhiambo R, et al. Evaluation of Kilifi epilepsy education programme: a randomized controlled trial. Epilepsia 2014; 55: 344-52.

54. Ajzen I. The theory of planned behavior. Organizational Behavior and Human Decision Processes 1991; 50: $179-211$.

55. Yew EHJ, Goh K. Problem-Based Learning: An Overview of its Process and Impact on Learning. Health Professions Education 2016; 2: 75-79.

56. Williams B, Pace AE. Problem based learning in chronic disease management: a review of the research. Patient Educ Couns 2009; 77: 14-9.

57. Escoffery C, Bamps Y, LaFrance WC, Jr., Stoll S, Shegog R, Buelow J, et al. Development of the Adult Epilepsy Self-Management Measurement Instrument (AESMMI). Epilepsy Behav 2015; 50: 172-83.

58. Nolte S, Elsworth GR, Newman S, Osborne RH. Measurement issues in the evaluation of chronic disease self-management programs. Qual Life Res 2013; 22: 1655-64.

59. Schwartz CE, Rapkin BD. Reconsidering the psychometrics of quality of life assessment in light of response shift and appraisal. Health Qual Life Outcomes 2004; 2: 16.

60. Heijmans M, Waverijn G, Rademakers J, van der Vaart R, Rijken M. Functional, communicative and critical health literacy of chronic disease patients and their importance for self-management. Patient Educ Couns 2015; 98: 41-8.

61. Rogers A, Kennedy A, Bower P, Gardner C, Gately C, Lee V, et al. The United Kingdom Expert Patients Programme: results and implications from a national evaluation. Med J Aust 2008;189: S21-4.

62. Epilepsie: Richtlijn voor diagnostiek en behandeling. In: Nederlandse Vereniging voor Neurologie; http://epilepsie.neurologie.nl/cmssite/index.php

63. Epilepsies: diagnosis and management. In: National Institue for Health and Care Excellence (NICE); https://www.nice.org.uk/guidance/cg137/chapter/1-Guidance\#coping -with-epilepsy

64. NICE. In: National Institure for Health and Care Escellence; https://www.nice.org.uk/process/pmg20/ chapter/introduction-and-overview\#information-about-this-manual

65. NHS. In: National Health Service UK; https://www.england.nhs.uk/commissioning/spec-services/keydocs/\#serv-dev

66. Bradley PM, Lindsay B. Care delivery and self-management strategies for adults with epilepsy. Cochrane Database Syst Rev 2008: CD006244.

67. Lorig KR, Ritter P, Stewart AL, Sobel DS, Brown BW, Jr., Bandura A, et al. Chronic disease self-management program: 2-year health status and health care utilization outcomes. Med Care 2001; 39: 1217-23.

68. Tielemans NS, Visser-Meily JM, Schepers VP, van de Passier PE, Port IG, Vloothuis JD, et al. Effectiveness of the Restore4Stroke self-management intervention "Plan ahead!": A randomized controlled trial in stroke patients and partners. J Rehabil Med 2015;47: 901-9.

69. Faber M, Harmsen M, van der Burg S, van der Weijden T.Gezamenlijke besluitvorming \&Zelfmanagement: Een literatuuronderzoek naar de effectiviteit en naar voorwaarden voor succes. In: Scientific Institute for 
Quality of Healthcare (IQ healthcare); https://www.raadrvs.nl/uploads/docs/Achtergrondstudie_ Gezamenlijkebesluitvorming_en_Zelfmanagement.pdf; 2013.

70. de Silva D. Evidence: Helping people help themselves-a review of the evidence considering whether is is worthwhile to support self-management. In: Health Foundation; http://www.health.org.uk/publication/ evidence-helping-people-help-themselves; 2011.

71. Campbell NC, Murray E, Darbyshire J, Emery J, Farmer A, Griffiths F, et al. Designing and evaluating complex interventions to improve health care. BMJ 2007; 334: 455-9.

72. Freedland KE, Mohr DC, Davidson KW, Schwartz JE. Usual and Unusual Care: Existing Practice Control Groups In Randomized Controlled Trials of Behavioral Interventions. Psychosomatic medicine 2011; 73 : 323-335.

73. Ayling K, Brierley S, Johnson B, Heller S, Eiser C. How standard is standard care? Exploring control group outcomes in behaviour change interventions for young people with type 1 diabetes. Psychol Health 2015;30: 85-103.

74. Kok G, Schaalma H, Ruiter RA, van Empelen P, Brug J. Intervention mapping: protocol for applying health psychology theory to prevention programmes. J Health Psychol 2004; 9: 85-98.

75. Lemmens KM, Nieboer AP, van Schayck CP, Asin JD, Huijsman R. A model to evaluate quality and effectiveness of disease management. Qual Saf Health Care 2008;17: 447-53. 



\section{Summary}

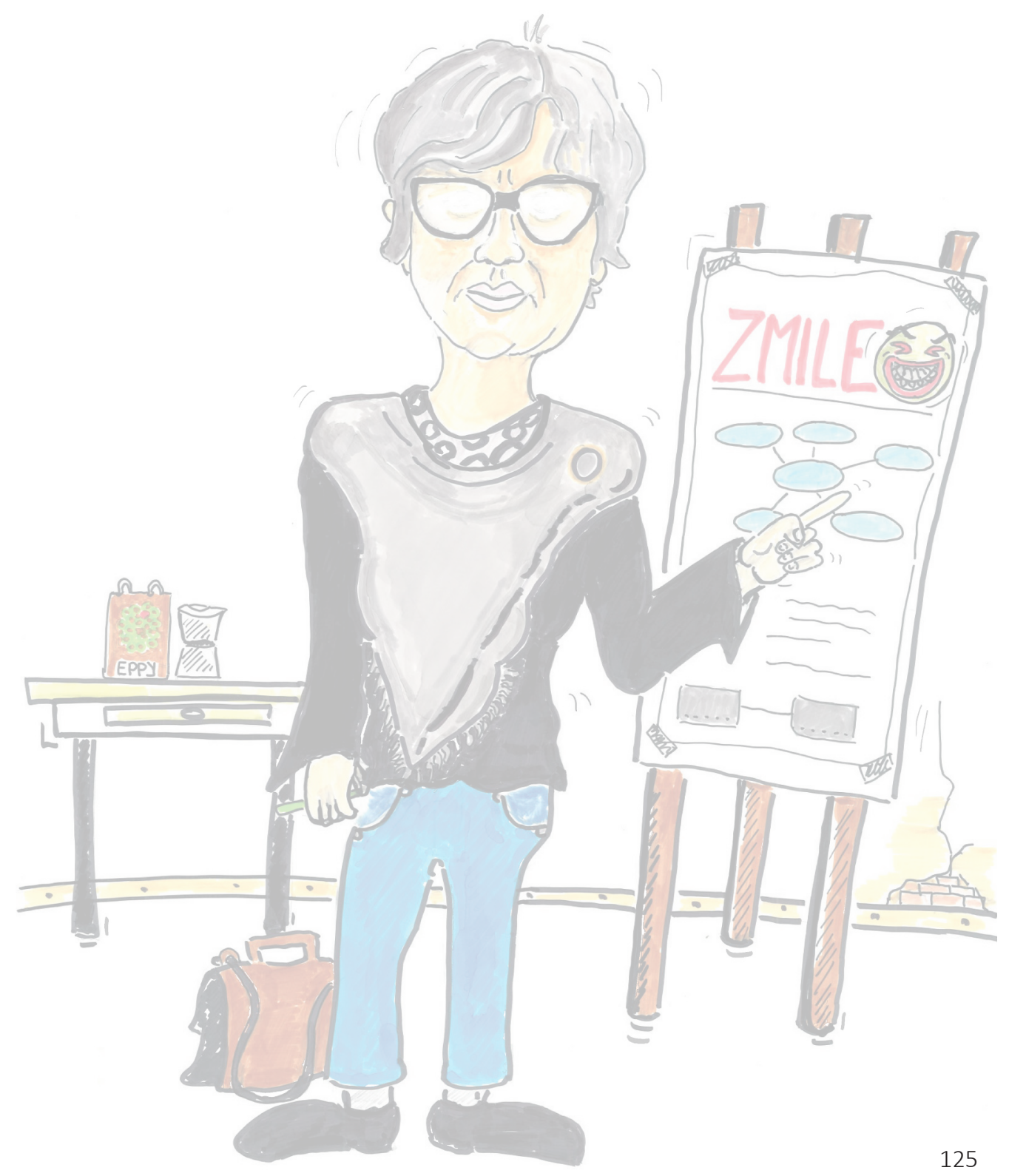




\section{SUMMARY}

Persons with epilepsy (PWE) have to manage many aspects of this neurological disease which is characterized by recurrent seizures resulting from sudden excessive electrical discharges in a group of brain cells. In addition to managing the medical aspects - the seizures, they have to adhere to treatment, such as anti-epileptic drugs, and deal with the psychological and emotional consequences of living with epilepsy, often resulting in a reduced quality of life (QOL). There is also an economic burden for PWE, such as diminished access to insurance and higher unemployment rates, and for society as a whole, in the form of health care costs and productivity losses.

Since most PWE live at home with their family, it is important for them to be able to manage their own lives. Dealing with the above-mentioned consequences of having a disease such as epilepsy, is a major task which requires specific skills. This focus on selfmanagement can also be found in the new definition of health: 'the ability to adapt and self-manage, in light of the physical, emotional and social challenges of life'. Self-management and self-management support is becoming increasingly important.

Self-management support programmes should include self-management education, but also peer-support, goal-setting skills, self-monitoring, and involvement in one's treatment plan.

Although there are promising self-management interventions for other chronic conditions, such as diabetes, rheumatoid and pulmonary conditions (i.e. the Chronic Disease Self-Management Programme (CDSMP)), self-management programmes for PWE are still in their infancy, especially in the Netherlands. We have, therefore, developed and evaluated a new self-management programme for PWE containing the above-mentioned necessary elements, and combining them into a multi-component intervention ( $\mathrm{MCl}$ ). In our study design, we incorporated recommendations from systematic reviews on the content of self-management interventions in the field of epilepsy and other chronic diseases, and performed a process evaluation and an economic evaluation alongside the clinical effectiveness study. Since eHealth is often used in self-management programmes, we added a survey to gain insight into the use of eHealth-by PWE. This thesis reports the results of the eHealth-study and of the ZMILE-study, the latter consisting of a process evaluation, and the (cost-) effectiveness study.

Chapter 1 provides background information about epilepsy, treatment options and the psychosocial and economic burden of epilepsy, both for PWE and for society as a whole. For PWE, as for their families, adapting to living with epilepsy is of great importance so that they can manage the disease themselves. After describing the terms self-management and self-management support, an explanation is provided of how the definition of these terms has influenced the development of the multi-component self-management intervention for adults with epilepsy, as shown in Figure 7.1. At the end of the chapter, there is an overview of the research questions answered in this thesis. 
Chapter 2 describes the design of the ZMILE-study (Zelf-Management Interventie Leven met Epilepsie). The overall objective of the ZMILE-study was to assess the (cost-) effectiveness and feasibility of a multi-component self-management intervention ( $\mathrm{MCl}$ ), aiming to improve self-efficacy and concordance in PWE. Concordance includes a consensual agreement about taking AEDs that has been established between patient and practitioner.

The $\mathrm{MCl}$ combines a self-management programme with eHealth interventions and is developed for adult patients with epilepsy and their relatives. The aim of the $\mathrm{MCl}$ is to enhance self-management skills, such as goal-setting and shared decision-making, as well as sharing information, i.e. risk-management and self-monitoring (by eHealth-tools).

This randomized controlled trial (RCT) evaluated the effectiveness of a nine-week epilepsy-specific self-management intervention (i.e. $\mathrm{MCl}$ ) by comparing the outcomes with a care as usual (CAU) group. One hundred PWE were randomly assigned to either the $\mathrm{MCI}$ or to CAU. Measurements were carried out at baseline and every three months; followup was at 12 months for the intervention group and six months for the CAU group. The primary outcome was self-efficacy, measured with the Epilepsy Self-Efficacy Scale (ESES). Secondary outcome measures were adherence, as a proxy for concordance, measured with the Medication Event Monitoring System (MEMS) and Medication Adherence Scale (MARS-5), general self-efficacy was measured with the General Self-efficacy scale (GSES), seizure frequency and severity by a questionnaire and National Hospital Seizure Severity Scale (NHS3), adverse anti-epileptic drug events were assessed by side-effects of Antiepileptic Drugs (SIDAED), depression and anxiety with the Hospital Anxiety and Depression Scale (HADS), proactive coping with the Utrecht Proactive Coping Competence (UPCC), and quality of life disease specific and general were measured with the Quality Of Life in Epilepsy (QOLIE-31P) and the EuroQol 5 dimensions 5 levels (EQ-5D-5L).

To determine cost-effectiveness, adherence, self-efficacy (Epilepsy Self-Efficacy Scale [ESES]), quality-adjusted life years (QALYs), healthcare costs, production losses and patient and family costs were assessed at baseline and during the 12-month study period. Incremental cost-effectiveness ratios (ICERs) (i.e., cost per increased adherence, self-efficacy, or QALY) and cost-effectiveness acceptability curves were calculated.

To establish whether the $\mathrm{MCl}$ was delivered according to protocol, and to examine the experience and opinions of participants and facilitators, the process evaluation used a mixed method design to collect both quantitative and qualitative data.

Chapter $\mathbf{3}$ discusses the results of the survey into the use of eHealth in PWE, performed among 1000 PWE of the out-patient clinic of Kempenhaeghe, who were asked to fill in a questionnaire. Since in self-management programmes, eHealth is an additional tool to support self-management, the main aim of this survey was to establish the role of eHealth in PWE. By using a mixed methods design, we explored how many PWE have access to computer or smartphone, which factors of epilepsy were monitored and how, and the perception of PWE about the use of eHealth-tools. The result of this study showed that 
PWE have less access to a computer or smartphone than the general population. They self-monitor more, but not necessarily by using eHealth-tools. Respondents perceived eHealth as an advantage in promoting self-care and health behaviour. A disadvantage was the fact that it could increase stress, by confronting PWE with their condition. PWE also mentioned concerns about privacy issues and the fact that eHealth probably cannot be used by everyone (i.e. the elderly or patients with a cognitive impairment).

Chapter 4 describes the findings of the process evaluation study. The goal of this study was to investigate if the $\mathrm{MCl}$ self-management intervention was implemented according to protocol, the attendance and adherence of patients and relatives, as well as assessing opinions of the patient, relatives and care professionals about the intervention. This evaluation was performed alongside the ZMILE-study, combining qualitative and quantitative data, which were collected by questionnaires and group interviews. Data were gathered from participants (i.e. 52 PWE and 37 relatives) and six facilitators. This study showed that all elements of the $\mathrm{MCl}$ were offered, with the exception of Eppy (an eHealth app), which was prematurely removed from the app stores. Attendance rate was high with $90 \%$ of the PWE attending five or more sessions. The participants regarded the sessions of the $\mathrm{MCl}$ overall as useful. Some suggestions were made for improvement, but in general, participants, relatives, facilitators and professionals viewed the $\mathrm{MCl}$ positively.

Chapter 5 reports on the results of the economic evaluation of the ZMILE-study. A trialbased economic evaluation was performed as part of a randomized controlled trial (RCT) in which the clinical effectiveness of the $\mathrm{MCl}$ was evaluated. The participants were randomly allocated to either the $\mathrm{MCI}$ group or CAU group. Follow-up period in the intervention group was 12 months and in the CAU group six months.

The economic evaluation demonstrates an improvement in adherence rates over 6 months of $75.9 \%$ for the intervention group compared to $63.7 \%$ for the CAU group. At the cost-effectiveness analysis, an ICER of $€ 54$ per point increase in ESES-score was found at 6 months' follow-up and a €1105 per point increase at 12 months' follow-upca. The cost-utility analysis showed an ICUR of €8,272 and €15,144 per QALY gained at 6- and 12months' follow-up, respectively. The $\mathrm{MCl}$ is cost-effective considering the present threshold of €50,000 per QALY gained.

Chapter 6 presents the result of the randomized controlled trial (RCT) assessing the effectiveness of the $\mathrm{MCl}$. The RCT compared the effectiveness of the $\mathrm{MCl}$ to CAU in adult patients with epilepsy. In total, 102 PWE participated in the trial, of whom 52 in the intervention group and 50 in the CAU group. The findings showed that the primary outcome measure, self-efficacy, was not significantly enhanced in the intervention group. Nevertheless, trends towards a beneficial effect of the $\mathrm{MCl}$ was seen in a significant decrease in anti-epileptic drug side-effects and in significant improvement in mood, daily activity and distress (i.e. domains of quality of life). Since both the process evaluation and the economic evaluation showed a more favourable outcome, we concluded that the $\mathrm{MCl}$ 
should be implemented, after adapting the suggested improvements. However, further research is recommended, alongside implementation, to establish if the recommended changes lead to additional effects of the $\mathrm{MCl}$, and if effects sustain over time.

Chapter 7 provides a general discussion of the research presented in this thesis, answers the research questions, and presents a more detailed discussion about the results of the ZMILE-study. The use of economic and process evaluations in addition to effectiveness studies on self-management interventions, as carried out in the ZMILE-study, gives a much broader perspective on the efficacy of an intervention than simply an RCT into clinical effectiveness. Including the information on the use of eHealth as a possible self-management tool by PWE, broadened this perspective even more. Suggestions from the intended users of self-management interventions in the eHealth study, the process evaluation, and additional study results have been included in the recommendations and suggestions for further research. These have been added to the discussion of the main findings.

In summary: in view of the positive results of the effect, process and economic evaluation, but also with regard to the positive reactions from the professionals, we recommend the further dissemination and implementation of the $\mathrm{MCl}$ in the clinical setting. 



\section{Samenvatting}

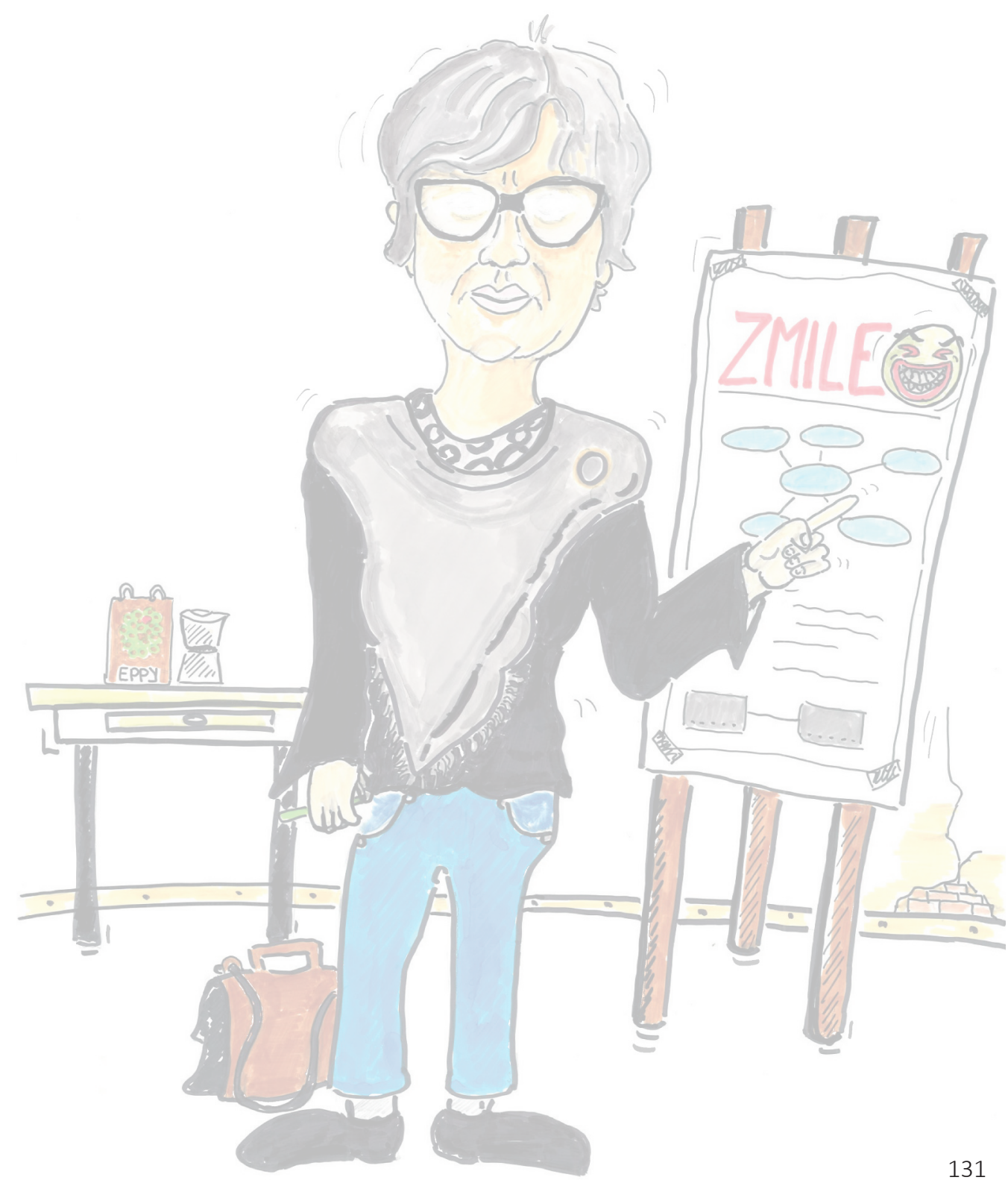




\section{SAMENVATTING}

Personen met epilepsie moeten meer dan alleen de medische aspecten van hun epilepsie managen. Epilepsie is een neurologische aandoening die wordt gekenmerkt door terugkerende aanvallen als gevolg van plotseling overmatige elektrische ontladingen in een groep hersencellen. Naast het managen van de medische aspecten, zoals de aanvallen en het volgen van behandelingen, zoals het gebruik van anti-epileptica, hebben personen met epilepsie te maken met de psychologische en emotionele gevolgen van het leven met epilepsie, met als gevolg een verminderde kwaliteit van leven. Daarnaast hebben personen met epilepsie ook last van economische gevolgen, zoals verminderde toegang tot verzekeringen en hogere werkloosheid. Economische gevolgen zijn er eveneens voor de samenleving als geheel, in de vorm van kosten voor gezondheidszorg en productiviteitsverlies.

Omdat de meeste personen met epilepsie gewoon thuis wonen is het belangrijk dat zij hun eigen leven kunnen leiden. Omgaan met de eerdergenoemde gevolgen van het hebben van epilepsie is een belangrijke opgave waarvoor specifieke (zelfmanagement) vaardigheden nodig zijn. Deze focus op zelfmanagement is ook terug te vinden in de nieuwe definitie van gezondheid: 'het vermogen om zich aan te passen en zichzelf te managen, in het licht van de fysieke, emotionele en sociale uitdagingen van het leven'. Zelfmanagement en zelfmanagementondersteuning wordt steeds belangrijker.

Interventies, die zelfmanagement ondersteunen zouden de volgende elementen moeten omvatten zelfmanagementeducatie, lotgenotencontact, vaardigheden aanleren om doelen te stellen, zelfmonitoren en betrokkenheid bij het eigen behandelplan stimuleren.

Hoewel er veelbelovende zelfmanagementinterventies zijn voor andere chronische aandoeningen, zoals diabetes, reumatoïde artritis en longaandoeningen, zoals bijvoorbeeld het Chronic Disease Self-Management Programme (CDSMP)), staat de ontwikkeling van zelfmanagementinterventies voor personen met epilepsie nog in de kinderschoenen, vooral in Nederland. We hebben daarom een nieuw zelfmanagementprogramma voor personen met epilepsie ontwikkeld in de vorm van een multi-component interventie $(\mathrm{MCl})$ waarin de bovengenoemde elementen gecombineerd worden.

In de ZMILE-studie waarin deze nieuwe zelfmanagement interventie wordt geëvalueerd, voeren we, zoals aanbevolen, naast een klinische effectiviteitsstudie ook een procesevaluatie en een economische evaluatie uit. Aangezien eHealth vaak gebruikt wordt in zelfmanagementprogramma's, ook in deze $\mathrm{MCl}$, hebben we een enquête toegevoegd om inzicht te krijgen in het gebruik van eHealth door personen met epilepsie. Dit proefschrift rapporteert de resultaten van deze studies.

Hoofdstuk 1 is een inleidend hoofdstuk dat achtergrondinformatie geeft over epilepsie, behandelingsmogelijkheden en de psychosociale en economische last van epilepsie, zowel voor personen met epilepsie als voor de samenleving als geheel. Zowel voor personen 
met epilepsie als voor hun families is aanpassing aan een leven met en het managen van epilepsie van groot belang. Na de beschrijving van de termen zelfmanagement en zelfmanagementondersteuning, wordt uitgelegd hoe deze begrippen tot de ontwikkeling van de multi-component zelfmanagementinterventie voor volwassenen met epilepsie hebben geleid, weergegeven in Figuur 7.1. Aan het einde van het hoofdstuk is er een overzicht van de onderzoeksvragen die in dit proefschrift worden beantwoord.

Hoofdstuk 2 beschrijft het studiedesign van de ZMILE-studie (Zelf-Management Interventie Leven met Epilepsie). Het doel van de ZMILE-studie is het beoordelen van de (kosten-) effectiviteit en haalbaarheid van een multi-component zelfmanagementinterventie $(\mathrm{MCl})$, gericht op het verbeteren van zelfeffectiviteit en concordantie in personen met epilepsie. De term concordantie refereert aan het bereiken van overeenstemming tussen patiënt en zorgverlener over de in te stellen therapie.

De $\mathrm{MCl}$ combineert een zelfmanagementprogramma met eHealth-interventies en is ontwikkeld voor volwassenen met epilepsie en hun naastbetrokkenen. Het doel van de $\mathrm{MCl}$ is het verbeteren van zelfmanagementvaardigheden door het stellen van doelen; zelfmanagement educatie over: gedeelde besluitvorming, risicomanagement en zelfmonitoring met behulp van eHealth-tools, evenals het delen van ervaringen over deze onderwerpen

Deze gerandomiseerde gecontroleerde studie (RCT) vergelijkt de (kosten) effectiviteit van een negen weken durende epilepsie-specifieke zelfmanagementinterventie $(\mathrm{MCl})$ met gebruikelijke zorg. Honderd personen met epilepsie worden willekeurig toegewezen aan ofwel de interventiegroep ofwel de controlegroep. Metingen werden uitgevoerd bij baseline en elke drie maanden; follow-up was na 12 maanden voor de interventiegroep en zes maanden voor de controlegroep. De primaire uitkomstmaat was zelfeffectiviteit, gemeten met de Epilepsy Self-Efficacy Scale (ESES). Secundaire uitkomstmaten waren therapietrouw, als een proxy voor concordantie, gemeten met het Medication Event Monitoring System (MEMS) en Medication Adherence Scale (MARS-5), algemene zelfeffectiviteit gemeten met de General Self-efficacy scale (GSES), aanvals-frequentie met een aanvalsvragenlijst, aanvalsernst gemeten met de National Hospital Seizure Severity Scale (NHS3), bijwerkingen van anti-epileptica in kaart gebracht met behulp van de SIDe-effects Anti-Epileptic Drugs (SIDAED), depressie en angst met de Hospital Anxiety and Depression Scale (HADS), proactieve coping met de Utrecht Proactive Coping Competence (UPCC), en kwaliteit van leven ziekte specifiek en algemeen gemeten met de Quality of Life in Epilepsie (QOLIE-31P) en de utiliteiten voor QALY met EuroQol (EQ-5D-5L).

Voor de kosteneffectiviteit worden adherentie, zelfeffectiviteit, voor kwaliteit gecorrigeerde levensjaren ( $Q A L Y$ 's), kosten voor gezondheidszorg, productieverliezen en kosten voor patiënt en gezin gemeten bij aanvang en gedurende de 12 maanden durende onderzoeksperiode. Incrementele kosteneffectiviteitsratio's (ICER's) (d.w.z. kosten per toegenomen therapietrouw, zelfeffectiviteit of $Q A L Y$ ), en kosteneffectiviteit acceptatie curves worden berekend. 
Via een procesevaluatie wordt, door middel van kwalitatieve en kwantitatieve dataverzameling, vastgesteld of de $\mathrm{MCl}$ volgens protocol wordt uitgevoerd, en worden ervaringen en meningen van deelnemers en facilitators onderzocht.

Hoofdstuk 3 bespreekt de resultaten van het onderzoek naar het gebruik van eHealth bij personen met epilepsie. Dit onderzoek wordt uitgevoerd onder 1000 personen met epilepsie van de polikliniek van epilepsiecentrum Kempenhaeghe, aan wie wordt gevraagd een vragenlijst in te vullen. Omdat in zelfmanagementprogramma's eHealth een extra hulpmiddel is om zelfmanagement te ondersteunen, is het belangrijkste doel van deze enquête om de rol van eHealth in personen met epilepsie vast te stellen. Met behulp van een mixed-method design wordt onderzocht hoeveel personen met epilepsie toegang hebben tot computer of smartphone, welke aanval uitlokkende factoren worden gemonitord en op welke manier, en de ervaringen en meningen van personen met epilepsie over het gebruik van eHealth-tools. Het resultaat van deze studie toont aan dat minder personen met epilepsie toegang hebben tot een computer of smartphone dan in de algemene bevolking. Ze monitoren meer gezondheidsgedrag, maar niet noodzakelijkerwijs door eHealth-tools te gebruiken. Respondenten zien eHealth als een voordeel bij het promoten van zelfzorg en gezondheidsgedrag. Als nadeel werd genoemd dat het stress kan vergroten doordat het bijhouden van aanvallen en aanval uitlokkend factoren confronterend kan zijn voor personen met epilepsie. Daarnaast wordt ook privacy en het feit dat eHealth niet door iedereen kan worden gebruikt (dat wil zeggen ouderen of patiënten met een cognitieve stoornis) als nadeel genoemd.

Hoofdstuk 4 beschrijft de bevindingen van de procesevaluatie. Het doel van deze studie was om te onderzoeken of de multi-component zelfmanagement interventie werd geïmplementeerd volgens protocol, in hoeverre patiënten en familieleden aanwezig en betrokken waren, evenals de meningen van de patiënt, familieleden en zorgprofessionals over de interventie. Deze evaluatie werd gelijktijdig uitgevoerd met de ZMILE-studie, waarbij kwalitatieve en kwantitatieve data werden gecombineerd, die werden verzameld door vragenlijsten en groepsinterviews. Gegevens werden verzameld van deelnemers aan de interventie (52 personen met epilepsie en 37 familieleden) en zes facilitators. Deze studie toonde aan dat alle elementen van de $\mathrm{MCl}$ werden aangeboden, met uitzondering van Eppy (een eHealth-app), die voortijdig werd verwijderd uit de app-stores. De aanwezigheid was hoog, $90 \%$ van de personen met epilepsie woonden vijf of meer sessies bij. De deelnemers beschouwden de sessies van de $\mathrm{MCl}$ over het algemeen als nuttig. Er werden enkele suggesties voor verbetering gedaan, maar over het algemeen zijn deelnemers, familieleden, facilitators en professionals positief over de $\mathrm{MCl}$.

Hoofdstuk 5 rapporteert de resultaten van de economische evaluatie van de ZMILE-studie. Deze economische evaluatie wordt uitgevoerd als onderdeel van een gerandomiseerde gecontroleerde trial (RCT) waarin de klinische effectiviteit van de $\mathrm{MCl}$ wordt onderzocht (zie hoofdstuk 6). De deelnemers worden willekeurig toegewezen aan de $\mathrm{MCl}$ 
of aan de controlegroep, die gebruikelijke zorg ontvangen. De follow-up periode in de interventiegroep is 12 maanden en in de controlegroep zes maanden.

De economische evaluatie laat een toename zien in adherentie ten opzichte van de controlegroep, over 6 maanden is de adherentie 63,7\% in de controlegroep en 75,9\% in de interventie ( $\mathrm{MCl}$ ) groep. Bij de kosteneffectiviteitsanalyse is de IKER €54 per punt verbetering op de self-efficacy (ESES) schaal bij 6 maanden en $€ 1.105$ per punt verbetering op 12-maanden follow-up. Bij de kosten-utiliteit liggen de incrementele kosteneffectiviteitsratios (IKERs) op €8.272 en €15.144 per gewonnen QALY op 6- en 12-maanden follow-up. De $\mathrm{MCl}$ is dus gelet op huidige grenswaarden voor QALY kosteneffectief.

Hoofdstuk 6 presenteert het resultaat van de gerandomiseerde gecontroleerde trial (RCT) die de effectiviteit van de $\mathrm{MCl}$ onderzocht. De RCT vergelijkt de effectiviteit van de $\mathrm{MCl}$ met een controlegroep met gebruikelijke zorg bij volwassen patiënten met epilepsie. In totaal namen 102 personen met epilepsie deel aan het onderzoek, waarvan 52 in de interventiegroep en 50 in de controlegroep. De resultaten laten zien dat de primaire uitkomstmaat, zelfeffectiviteit, niet significant verhoogd is in de interventiegroep. In de effectstudie vinden we wel een verbetering op de bijwerkingenschaal (SIDAED) en op een aantal kwaliteit van leven schalen van de QOLIE-31P ten gunste van de ZMILE-interventie $(\mathrm{MCl})$ groep.

Omdat zowel daarnaast ook de procesevaluatie als de economische evaluatie een gunstige uitkomst laat zien, concludeerden we dat de $\mathrm{MCl}$ zou moeten worden geïmplementeerd, na aanpassing van de voorgestelde verbeteringen. Naast implementatie wordt verder onderzoek aanbevolen om vast te stellen of de aanbevolen wijzigingen leiden tot aanvullende effecten van de $\mathrm{MCl}$ en of deze effecten in de loop van de tijd aanhouden.

Hoofdstuk 7 bediscussieert het onderzoek gepresenteerd in dit proefschrift, beantwoordt de onderzoeksvragen en presenteert een meer gedetailleerde discussie over de resultaten van de ZMILE-studie. Het gebruik van economische en procesevaluaties naast effectiviteitsstudies over zelfmanagementinterventies, zoals uitgevoerd in de ZMILE-studie, geeft een veel breder perspectief op de effectiviteit van een interventie dan alleen een RCT naar klinische effectiviteit. Het resultaat van het onderzoek naar het gebruik van eHealth als een mogelijk zelfmanagementtool voor personen met epilepsie, heeft dit perspectief nog meer verbreed. Suggesties van de beoogde gebruikers van zelfmanagementinterventies in de eHealth-studie, de procesevaluatie en aanvullende studieresultaten zijn opgenomen in de aanbevelingen en suggesties voor verder onderzoek.

Samenvattend: gelet op de positieve resultaten in de effect-, proces- en economische evaluatie, maar ook lettend op de positieve reacties uit de praktijk bevelen we verdere verspreiding en implementatie van de $\mathrm{MCl}$ in de klinische setting aan. 



\section{Valorisation}

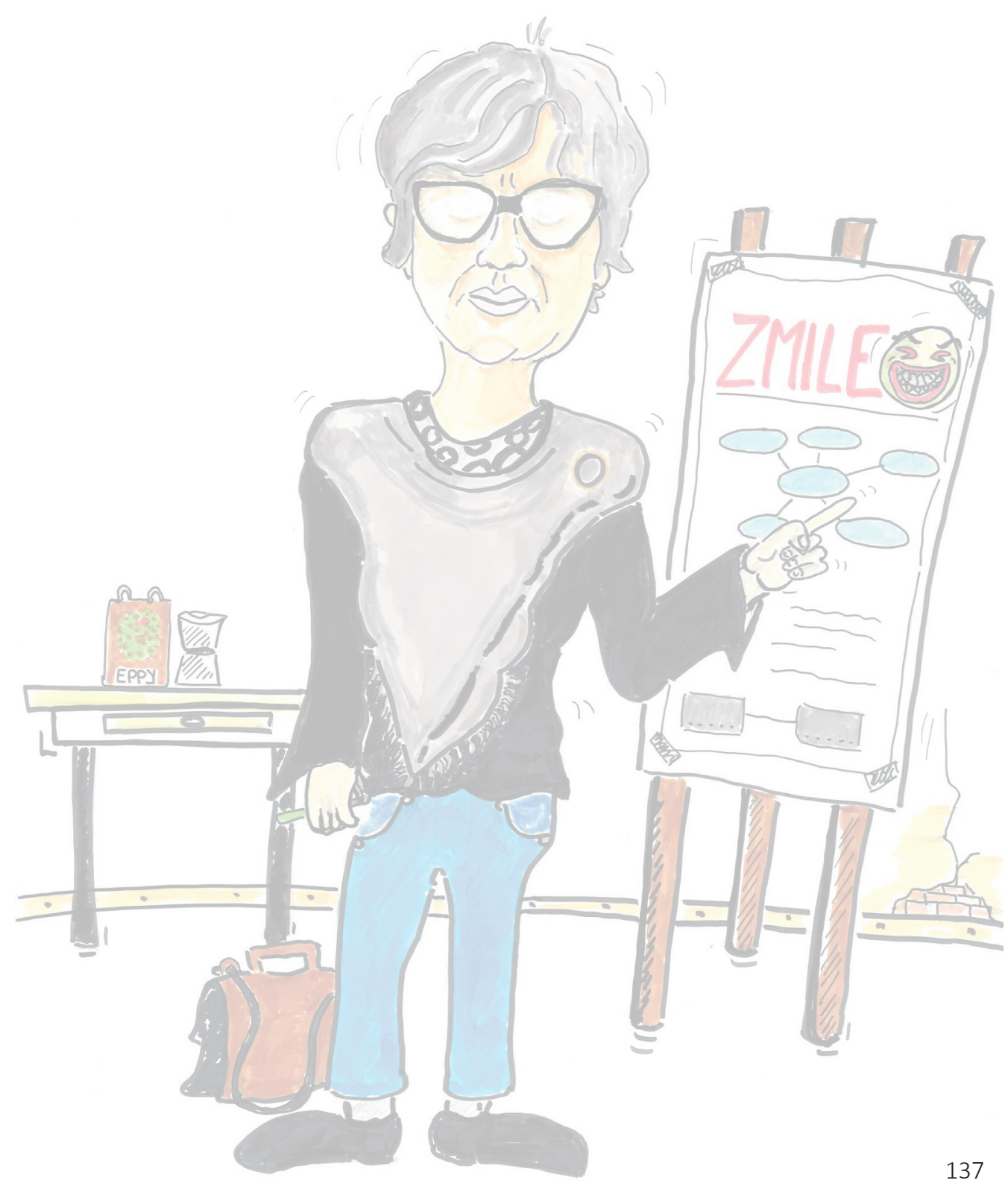




\section{VALORISATION}

Epilepsy is an invasive disease; not only does it have a psychosocial impact on persons with epilepsy (PWE) and their relatives, but it also has an economic impact on society as a whole. The costs involved include direct health care (e.g. health care visits, hospitalisation), informal care by family, friends or the community, indirect costs due to productivity losses and unemployment, and societal costs. The latter are the result of the effect that epilepsy, or epilepsy treatment, has on productivity and on independent living.

From the perspective of PWE and their relatives, as well as from a societal perspective it would seem valuable to achieve an efficient patient-centred health-care system, focussing on optimal self-management and quality of life. Self-management is defined as the ability to deal with the symptoms, medical treatment, lifestyle changes, and physical and psychosocial consequences.

In 2017, the National Health Care Institute started a programme called 'appropriate care' that focusses on assessing whether diagnostics and (therapeutic) interventions are being applied in a patient-oriented, effective and cost-effective manner. Part of this programme, which was setup together with patients, healthcare professionals and insurance companies, was a project that investigated whether the care of PWE was deployed in this way. It was concluded that the psychosocial care of PWE (and their relatives) is lacking, and that a psychosocial self-management intervention could be effective. The subject of this thesis is relevant in that it provides an evidence-based intervention, introducing psychosocial and self-management support not only for PWE, but also for their relatives. The multi-component intervention $(\mathrm{MCl})$, which is a novel self-management intervention in the Netherlands for PWE, provides support in the development of self-management skills. To increase these skills, different strategies are applied, such as self-management education, setting goals and increasing one's involvement in the treatment plan, thus leading to a shared treatment decision and self-monitoring.

The positive results of the effectiveness study, the process and economic evaluation of this thesis, mean that further dissemination and implementation of the adapted selfmanagement intervention $(\mathrm{MCl})$ in the clinical setting is possible.

\section{RELEVANCE FOR STAKEHOLDERS}

The results of this thesis are of relevance for patients and their relatives, healthcare professionals, insurance companies and policy makers (local and nationally)

\section{Patients \& Relatives}

Patients and their relatives will profit from this research, as it emphasizes the importance of their perspective on self-management and of how they perceive self-management support. From the literature we know that there is no such thing as the typical chronic patient and there is no 'one size that fits all', knowledge that is supported by this thesis. 
To achieve an efficient patient-centred health care system, the entire system should be considered, since the demand on informal care is growing. Relatives will benefit from this research, because it emphasizes their need for support from health care professionals

\section{Researchers}

The studies described in this thesis are relevant for a broad range of researchers in the field of epilepsy and economic evaluations. Since this thesis represents the first study to look at/consider a self-management intervention for PWE in the Netherlands, many aspects of self-management (support) have not yet been investigated. For instance, what is the implication if instead of finding a significant improvement on the primary outcome (self-efficacy), significant results are found on secondary outcomes (QOL, side-effects).

The $\mathrm{MCl}$ is also innovative in that it includes relatives. They are stimulated to focus on their own goals, experiences and problems, which are an inherent part of living with a relative with epilepsy. The introduction of relatives within the $\mathrm{MCl}$, disclosed a knowledge cap about the results relatives hope to achieve by attending an intervention and research on this subject.

The challenge for researchers in economic evaluations is to develop an instrument to measure quality of life, which will be accepted in the guidelines for economic evaluations, and which does not have the limitations of the EuroQOL-5D-5L.

\section{Healthcare professionals}

Healthcare professionals (neurologists, nurse practitioners, etc.) working with PWE can benefit from the study findings. Our study showed that personalized goalsetting of PWE, alongside personalized information and shared decision-making, plays an important role in their psychosocial functioning. By taking these factors into account, healthcare professionals will be able to support patients in achieving self-management, either by means of individual interventions or by the $\mathrm{MCl}$, the latter including group sessions.

As well as improving the quality of care for PWE, the effect of stimulating self-management behaviour may also result in more efficient consultations with the healthcare provider.

In contrast to, for instance, the guideline from the United Kingdom (The National Institute for Health and Care Excellence (NICE) Epilepsies: diagnosis and management CG137), self-management and self-management support do not feature in the current national epilepsy guideline. As pointed out in the discussion (chapter 7), findings of the studies can be used to update the Dutch national epilepsy guideline to include these aspects.

\section{Policy makers \& Healthcare insurance companies}

Both studies convey an important message to policy makers and insurance companies; they have to make a deliberate choice about what to reimburse. 
Over the past decade, policy makers and insurance companies have emphasized the need for patients with a chronic condition to take responsibility in managing their own condition and for informal caregivers to play a larger role. Self-management, especially self-management using eHealth, was seen as a favourable option. Strategies to improve self-management in this way are based on research on chronic diseases, such as diabetes, COPD etc., and not on research on more vulnerable groups such as epilepsy, a disease known for the effect on cognition and the accompanying challenges for PWE.

The ZMILE-study demonstrated favourable cost-effectiveness ratios, which may create a positive attitude to the self-management intervention for adults with epilepsy. This thesis also offers an insight into the feasibility of this self-management intervention as perceived by PWE, relatives and healthcare professionals. In addition, our research showed that a group of PWE did not have access to or the ability to use eHealth, meaning this option is not appropriate for a subcategory of patients. The knowledge may be useful for policy makers and insurance companies in their approach to self-management for special groups.

Table 10.1: Overview of planned and realized products and activities per target group

\begin{tabular}{|c|c|c|c|}
\hline Target group & Products \& Activities & Realized locally & $\begin{array}{l}\text { Realized } \\
\text { nationally in } \\
2019\end{array}$ \\
\hline \multirow[t]{5}{*}{ Patients \& relatives } & $\mathrm{MCl}$ & $x$ & $x$ \\
\hline & 5-step framework (adapted) & $x$ & $x$ \\
\hline & Manual - facilitators & $x$ & $x$ \\
\hline & workgroup 'national accessibility' & & $x$ \\
\hline & Project group - business case & & $x$ \\
\hline \multirow[t]{5}{*}{ Healthcare professionals } & Presentations & $x$ & $x$ \\
\hline & Scientific articles & $x$ & \\
\hline & workgroup 'national accessibility' & & $x$ \\
\hline & Project group - business case & & $x$ \\
\hline & National education organizations & $x$ & $x$ \\
\hline \multirow[t]{4}{*}{ Insurance companies } & Scientific articles & $x$ & \\
\hline & $\begin{array}{l}\text { Information on costs (thesis Ben Wijnen and } \\
\text { Loes Leenen) }\end{array}$ & $x$ & \\
\hline & $\begin{array}{l}\text { Information on patient \& relatives } \\
\text { perspective (process evaluation) }\end{array}$ & $x$ & \\
\hline & Project group - business case & & $x$ \\
\hline \multirow[t]{3}{*}{ Researchers } & Scientific articles & $x$ & $x$ \\
\hline & presentations & $x$ & $x$ \\
\hline & Evaluation implementation & & $x$ \\
\hline \multirow[t]{7}{*}{ Policy makers (including local) } & $\begin{array}{l}\text { Information on costs (thesis Ben Wijnen and } \\
\text { Loes Leenen) }\end{array}$ & $x$ & \\
\hline & Scientific articles & $x$ & \\
\hline & workgroup 'national accessibility' & & $x$ \\
\hline & Business cases (local) & $x$ & $x$ \\
\hline & Project group - business case & & $x$ \\
\hline & Business case (national) & & $x$ \\
\hline & Project plan & $x$ & \\
\hline
\end{tabular}




\section{DISSEMINATION \& IMPLEMENTATION}

The $\mathrm{MCl}$ was designed and validated. Based on recommendations of PWE and relatives, and considering the positive reactions from practitioners (chapter 4 ), the adapted intervention is ready for implementation.

As table 10.1 shows, some activities and products have already been realized and some are planned within the dissemination and implementation process. Products and/or activities focus on three levels of stakeholder:

- At a micro level: end-users (i.e. healthcare professionals, patients and relatives).

- At a meso level: managers and policy makers (e.g. insurance companies etc.).

- At a macro level: umbrella organizations (professional associations, patient support groups, national knowledge networks and national committees).

The products and activities, planned and realized per target group, are summarized in table 10.1.

\section{Realized locally}

In the design phase of the ZMILE-study, and of the multi-component self-management intervention $(\mathrm{MCl})$, an advisory board was selected, with valorisation in mind. This board, which consisted of patient representatives, healthcare professionals, members of industry and representatives of healthcare insurances, met three or four times a year.

The results of studies were also presented on three levels:

- At a micro level: by presenting results at Maastricht University Medical Centre (vakgroep neurologie), Maastricht 2017, and in a SepiON (Stichting Epilepsie Onderwijs Nederland) course on self-management, Hoevelaken 2018. (i.e. health care professionals)

- At a meso level: by publication in international and national journals (e.g. researchers)

- At a macro level: Presentations at national and international conferences. Examples of national conferences were the $17^{\text {th }}$ and $20^{\text {th }}$ edition of the annual international clinical symposium Kempenhaeghe, Heeze 2015 and 2018. International conferences were the $32^{\text {nd }}$ International Epilepsy Congress, Barcelona 2017, the $3^{\text {rd }}$ Congress of the European Academy of Neurology, Amsterdam 2017 and the 10 ${ }^{\text {th }}$ ICN NP/APN Conference, Rotterdam 2018.

\section{Planning for realisation nationally in 2019}

As the intention is to implement the $\mathrm{MCl}$ in the regular care of PWE, a dissemination and implementation plan has been designed, in collaboration with various stakeholders.

The four main objectives of the dissemination and implementation plan are:

1. To optimize the $\mathrm{MCl}$

2. To secure $\mathrm{MCl}$ financially and practically

3. T make the $\mathrm{MCl}$ accessible nationally (and exploring international opportunities)

4. To evaluate and produce a Business Case 
To achieve these objectives in current practice, the following mix of activities / strategies are planned:

To further optimize the $\mathrm{MCl}$ by:

- Adjusting the $\mathrm{MCl}$ based on the process evaluation

- Professionalizing the available course material in terms of layout and practical applicability;

- Developing the training for course leaders, so that it is easier to implement the course at different locations;

Financially securing the $\mathrm{MCl}$ by:

- Remaining financially accessible for participants of the $\mathrm{MCl}$;

- Composing a project team; that includes the representation of patients with epilepsy, health insurers, pharmaceutical companies and both regional and national healthcare organizations in order to prepare a business case.

- Drawing up a business case, in which the costs and benefits are calculated for the various parties

Practically securing and making the $\mathrm{MCl}$ accessible on a national scale by:

- Drawing up a communication plan for optimal dissemination;

- Informing stakeholders about the $\mathrm{MCl}$ (presentations and publications);

- Aiming at involvement in the 'landelijk leerhuis epilepsie' and SepiON at an educational level;

- Aiming at organizational change, so that the $\mathrm{MCl}$ is a standard part of the care of epilepsy (participation by end-users and professionals);

Evaluating by

- Monitoring the process and results with regard to all objectives

The intention is to disseminate the $\mathrm{MCl}$ nationally in 2019; as a first step in this process, the $\mathrm{MCl}$ will be offered to PWE and their relatives at the Medical University Centre Maastricht (MUMC) in the autumn of 2018. 
Addendum

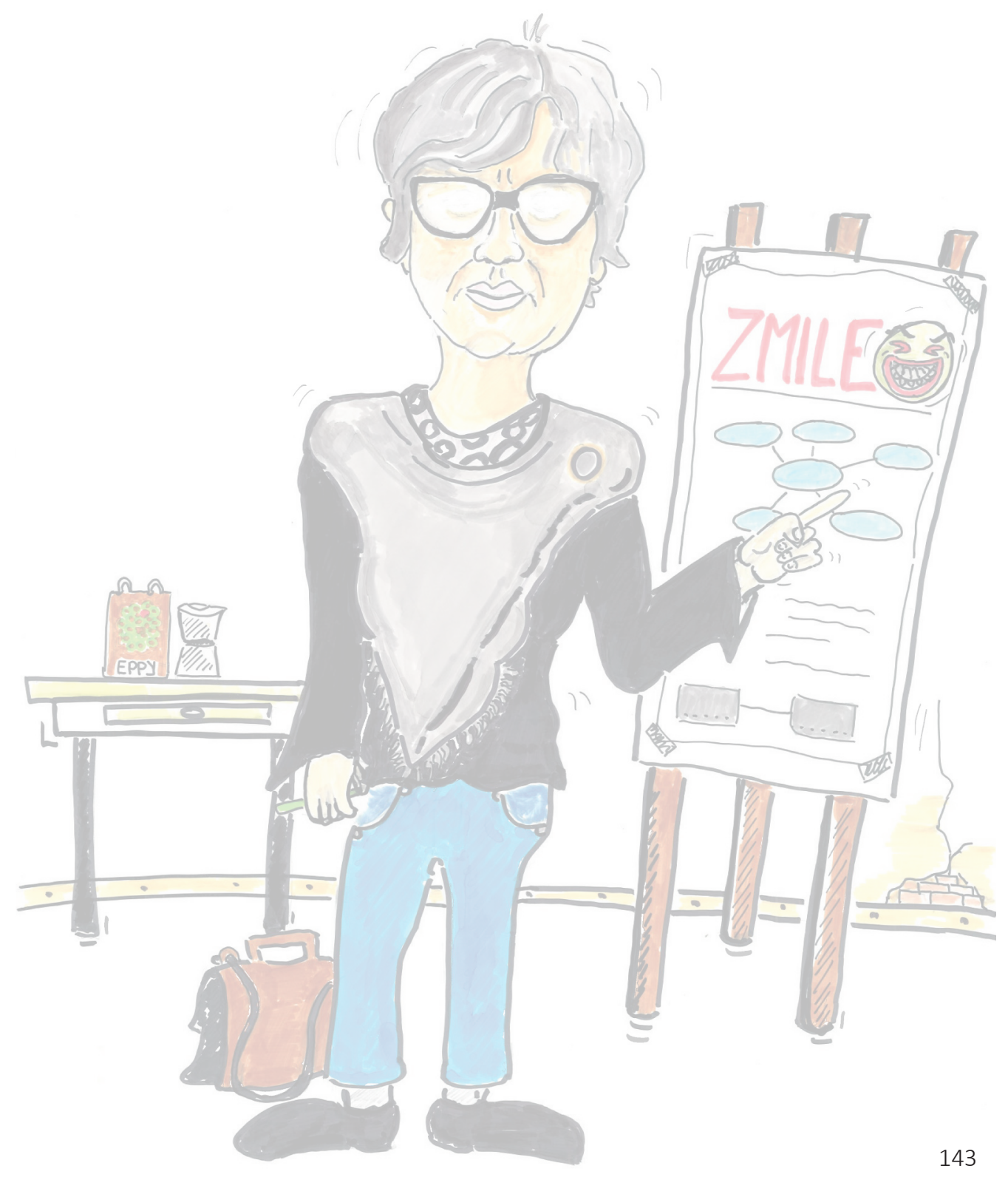




\section{LIST OF PUBLICATIONS}

\section{In this Thesis}

Leenen LAM, Wijnen BFM, Kessels AGH, Chan $H$, de Kinderen RJA, Evers SMAA, van Heugten CM Majoie HJM. Effectiveness of a multicomponent self-management intervention for adults with epilepsy (ZMILE study): A randomized controlled trial. Epilepsy Behav 2018;80: 259-265.

Leenen LAM, Wijnen BFM, van Haastregt JCM, de Kinderen RJA, Evers SMAA, Majoie HJM, van Heugten CM. Process evaluation of a multi-component self-management intervention for adults with epilepsy (ZMILE study). Epilepsy Behav 2017;73: 64-70.

Wijnen BFM, Leenen LAM, de Kinderen RJA, van Heugten CM, Majoie M, Evers S. An economic evaluation of a multicomponent self-management intervention for adults with epilepsy (ZMILE study). Epilepsia 2017;58: 1398-1408

Leenen LA, Wijnen BF, de Kinderen RJ, van Heugten CM, Evers SM, Majoie MH. Are people with epilepsy using eHealth-tools? Epilepsy Behav 2016;64: 268-272.

Leenen, LA, Wijnen BF, de Kinderen RJ, Majoie MH, van Heugten CM, Evers SM. (Cost)effectiveness of a multi-component intervention for adults with epilepsy: study protocol of a Dutch randomized controlled trial (ZMILE study). BMC Neurol 2014;14: 255

\section{Peer-reviewed International Publications}

Klinkenberg S, van den Borne CJH, Aalbers MW, Verschuure P, Kessels AG, Leenen L, Rijkers K, Aldenkamp AP, Vles JS, Majoie HJ. The effects of vagus nerve stimulation on tryptophan metabolites in children with intractable epilepsy. Epilepsy Behav 2014;37: 133-138.

Klinkenberg S, Aalbers MW, Vles JS, Cornips EM, Rijkers K, Leenen L, Kessels FG, Aldenkamp AP, Majoie M. Klinkenberg et al. reply. Dev Med Child Neurol 2013;55: 1956.

Klinkenberg S, van den Bosch CN, Majoie HJ, Aalbers MW, Leenen L, Hendriksen J, Cornips E, Rijkers K, Vles JS, Aldenkamp AP. Behavioural and cognitive effects during vagus nerve stimulation in children with intractable epilepsy - a randomized controlled trial. Eur J Paediatr Neurol 2013;17: 82-90.

Vijgen GH, Bouvy ND, Leenen L, Rijkers K, Cornips E, Majoie M, Brans B, van Marken Lichtenbelt WD. Vagus nerve stimulation increases energy expenditure: relation to brown adipose tissue activity. PLoS One, 2013;8: e77221 
Klinkenberg S, Aalbers MW, Vles JS, Cornips EM, Rijkers K, Leenen L, Kessels FG, Aldenkamp AP, Majoie M. Vagus nerve stimulation in children with intractable epilepsy: a randomized controlled trial. Dev Med Child Neurol 2012;54: 855-61.

Klinkenberg S, Majoie HJ, van der Heijden MM, Rijkers K, Leenen L, Aldenkamp AP. Vagus nerve stimulation has a positive effect on mood in patients with refractory epilepsy. Clin Neurol Neurosurg 2012;114: 336-40.

Bootsma HP, Vos AM, Hulsman J, Lambrechts D, Leenen L, Majoie M, Savelkoul M, Schellekens A, Aldenkamp AP. Lamotrigine in clinical practice: long-term experience in patients with refractory epilepsy referred to a tertiary epilepsy center. Epilepsy Behav 2008;12: 262-8.

Bootsma HP, Ricker L, Diepman L, Gehring J, Hulsman J, Lambrechts D, Leenen L, Majoie M, Schellekens A, de Krom M, Aldenkamp AP et al. Long-term effects of levetiracetam and topiramate in clinical practice: A head-to-head comparison. Seizure 2008;17: 19-26.

Bootsma HP, Ricker L, Diepman L, Gehring J, Hulsman J, Lambrechts D, Leenen L, Majoie M, Schellekens A, de Krom M, Aldenkamp AP. Levetiracetam in clinical practice: long-term experience in patients with refractory epilepsy referred to a tertiary epilepsy center. Epilepsy Behav 2007;10: 296-303.

Bootsma HP, Aldenkamp AP, Diepman L, Hulsman J, Lambrechts D, Leenen L, Majoie M, Schellekens A, de Krom M. The Effect of Antiepileptic Drugs on Cognition: Patient Perceived Cognitive Problems of Topiramate versus Levetiracetam in Clinical Practice. Epilepsia 2006;47 Suppl 2: 24-7.

Roberts GM, Majoie HJ, Leenen LA, Bootsma HP, Kessels AG, Aldenkamp AP, Leonard BE. Ketter's hypothesis of the mood effects of antiepileptic drugs coupled to the mechanism of action of topiramate and levetiracetam. Epilepsy Behav 2005;6: 366-72.

Bootsma HP, Coolen F, Aldenkamp AP, Arends J, Diepman L, Hulsman J, Lambrechts D, Leenen L, Majoie M, Schellekens A, de Krom M. Topiramate in clinical practice: long-term experience in patients with refractory epilepsy referred to a tertiary epilepsy center. Epilepsy Behav 2004;5: 380-7.

Aldenkamp AP, Arends J, Bootsma HP, Diepman L, Hulsman J, Lambrechts D, Leenen L, Majoie M, Schellekens A, de Vocht J. Randomized double-blind parallel-group study comparing cognitive effects of a low-dose lamotrigine with valproate and placebo in healthy volunteers. Epilepsia 2002;43: 19-26.

\section{Peer-reviewed National Publications}

Wijnen B, Leenen L, de Kinderen R, van Heugten CM, Evers S, Majoie M. Kosteneffectiviteit van zelfmanagement-interventie voor volwassenen met epilepsie. Epilepsie, periodiek voor professionals 2017; 15(3),:17-19 
Leenen, L., Wijnen, B., de Kinderen, R., van Heugten, C.M., Evers, S., Majoie, M. Het gebruik van e-health door mensen met epilepsie. Epilepsie, periodiek voor professionals 2017; 15(3): 14-16

Wijnen B, Majoie M, van Heugten C.M, de Kinderen R, Leenen L, Evers S. Economische Evaluatiestudies binnen de epilepsie. Epilepsie, periodiek voor professionals 2015; 2(13): 16-20

Majoie M, Hunsel van F, Leenen L, Bootsma HP, Kamping A, Puyenbroek van E. Melden van Bijwerkingen van Anti-epileptica. Epilepsie, periodiek voor professionals 2009;7(1).

\section{Abstracts}

Wijnen BFM, Leenen LAM, de Kinderen RJA, van Heugten CM, Majoie M, Evers S. An economic evaluation of a multicomponent self-management intervention for adults with epilepsy (ZMILE study). Abstracts. Epilepsia 2017;58: S11

Chan HY, Wijnen B, Leenen L, de Kinderen R, Majoie M, Evers S. A comparison of the Dutch assessment of Quality of Life-8D (AQoL-8D), the QOLIE-31p and the EQ-5D-5L in epilepsy European Journal of Neurology 2017 Vol. 24, p.468-468.

Wijnen BFM, Leenen LAM, de Kinderen RJA, Majoie MH, van Heugten CM, Evers SMAA. (Cost)-effectiveness of a multi-component intervention for adults with epilepsy: study protocol of a Dutch randomized controlled trial (ZMILE study). Value Health 2014;17(7):A582-A583

\section{Dutch Papers}

Loes Leenen. Neem de Hoofdrol in je leven. Puls, uitgave Epilepsie Vereniging Nederland Leenen LAM. Aanvalskalender: digitaal en strikt persoonlijk. De ontwikkeling van een digitale aanvalskalender, als instrument voor zelfmanagement bij patiënten met epilepsie 2009; MANP. (https://hbo-kennisbank.nl/record/share_kithu/oai:surfshare_ kit.nl :e4e745de-2e9e-44e8-9e28-102e4716379b) 


\section{DANKWOORD}

'Het leven is als fietsen:

Om je evenwicht te houden moet je blijven bewegen'

(Einstein)

Het promotietraject was mooi, soms zwaar, maar om in evenwicht te blijven moest ik wel blijven bewegen. Gelukkig was het altijd uitdagend. Voordat ik met dit hoofdstuk een periode afsluit wil ik eerst alle mensen bedanken die mij in deze periode gesteund en soms ondersteund hebben.

Als eerste wil ik de patiënten en hun naasten bedanken. Dit onderzoek kon alleen worden gedaan door hun inzet. Zij volgden de zelfmanagementcursus en vulden de vragenlijsten in. Het waren veel vragenlijsten en dit herhaalde zich om de paar maanden. Toch bleven zij enthousiast, wat ik ook later terugzag als ik hen tegen kwam op de polikliniek.

Ik wil graag de leden van de beoordelingscommissie van dit proefschrift: prof. dr. M.E. de Vugt, prof. dr. P.A.J.M. Boon, prof. dr. C.G. Faber, prof. dr. W.J.M. Scholte op Reimer en dr. A.T.G. Paulus, bedanken voor het lezen en beoordelen van mijn thesis.

Een speciaal woord van dank voor diegenen die mijn promotie begeleid hebben. Allereerst prof. dr. H.J.M. Majoie. Beste Marian, ik had mijn masterdiploma nog geen half uur in mijn bezit toen jij in de aula van de Hogeschool Utrecht al heel enthousiast riep "En nu promoveren". Hoewel ik niet direct kon starten, bleef je stimuleren. Ook tijdens het gehele promotietraject was je heel belangrijk voor mij. Je gaf me steun toen ik moest leren dat een 'oude rot' in het vak heel goed samengaat met een jonge onderzoeker. Je maakte ruimte in je agenda als ik weer eens met vragen worstelde. Ik weet niet of ik zonder die ondersteuning mijn evenwicht wel had kunnen behouden. Dank daarvoor.

Prof. dr. mr. S.M.A.A. Evers en prof. dr. C.M. van Heugten, Silvia en Caroline, jullie waren onmisbaar, maar niet alleen voor het slagen van dit onderzoek. Silvia, jouw tomeloze enthousiasme en je vroege mails met correcties hielpen me de anders nutteloze tijd in de trein door te komen. Caroline, jouw precisie, heeft voor eenduidigheid gezorgd en jouw vragen om verheldering hebben mij helderheid gegeven. Jullie hielpen mij niet alleen buiten de grenzen van mijn vak te kijken, maar ook naar mezelf als onderzoeker. Dank daarvoor.

Alle drie MIJN professoren hebben verschillende unieke kwaliteiten en hoewel verschillen soms kunnen leiden tot problemen, leverde voor mij 1+1+1 veel meer op dan 3, ondanks dat ik dat niet statistisch kan onderbouwen.

Over statistiek gesproken, Ben Wijnen, bij mij was dat heel roestig en zeker een beetje gedateerd, maar jij wist in mini-colleges mijn statistiek te updaten. Je was niet alleen mijn steun en toeverlaat op het gebied van statistiek maar stond ook altijd klaar om me te helpen en te ondersteunen als ik het even niet meer zag zitten. Je wist altijd wel een gaatje in je agenda te vinden. We hebben samen een mooi traject doorlopen en naast 
dat we straks beiden gepromoveerd zijn, jij al een jaartje eerder, heb ik er vooral een vriend aan over gehouden. Een vriendschap die zich uitbreidde naar Hoi Yau. Zij verlichtte mijn werkdruk enorm, anders zat ik misschien nu nog data in te voeren.

Ja, data invoeren, ook jij Etienne was daarbij belangrijk toen we honderden enquêtes moesten invoeren. Zonder jouw hulp was ik misschien nooit zo ver gekomen.

Er komt heel wat kijken bij het ontwikkelen van een cursus of het onderzoeken ervan. Om alles leesbaar te maken heb ik veel steun gehad. Dank hiervoor, Caroline van Heugten, Caroline Caron, Madeleine van Kan, Miriam Brennand en al die anderen die input hebben gegeven op de inhoud. Janne Lambermont, speciale dank voor de mooie lay-out van de vragenlijsten. Je hebt er veel tijd ingestopt. Brenda Vollers, na het corrigeren van mijn Engelstalige stukken heb ik vaak het idee gehad, 'nu staat er wat ik wil zeggen!'. Dank daarvoor en jouw correcties hebben ook mijn Engels verbeterd. De afdeling Communicatie van Kempenhaeghe, was van onschatbare waarde voor het behalen van het streefaantal patiënten in de studie, dank Marianne.

Verder wil ik de adviesgroep bedanken. Zij waren betrokken gedurende het gehele ZMILE-project: Caroline Caron, Madeleine van Kan, Arjan Wietses, Loes Hageman, Anouk Pieron, Bernard Vrijens, Anja Lenssen, Karin Scheele, Frances Tan, Richard Lazeron en Christian Vader.

Ook prof. dr. Paul Boon en prof. dr. Bert Aldenkamp, wil ik bedanken. Vanuit jullie verantwoording voor Onderzoek \& Ontwikkeling binnen Kempenhaeghe, leggen jullie de lat hoog. Als promovendus moet je elk half jaar een samenvatting geven van het verloop en de resultaten van het onderzoek. De kritische vragen die volgen bereiden je goed voor op het einddoel.

De kern van het ZMILE-onderzoek was de cursus en hoe draai je nu een cursus zonder cursusleiders. Carly en Bea, jullie waren cursusleiders van het eerste uur. Joke, Peter en Marion vulden al snel de gaten in de planning en zonder de inzet van jullie allemaal had ik het niet gered. Jeske, mijn maatje in Maastricht, je was nog bezig met je opleiding tot verpleegkundig specialist en toch was je bereid in je vrije tijd de cursus te geven. We hebben veel lol gehad samen, vooral als je met een van de cursisten weer eens in het dialect aan het 'kallen' was en ik daar overduidelijk geen snars van begreep. 'Kallen' voor de niet-Limburgers onder ons betekent praten en dat is het enige Limburgse woord dat ik wel begrijp.

Niet alleen door het geven van de cursus kreeg ik jullie steun. Dat jullie trots waren omdat een van jullie in een promotietraject zat, heeft me regelmatig dat steuntje in de rug gegeven dat nodig was om door te zetten. Die interesse voelde ik ook bij mijn collega's uit Oosterhout: Mieke, Ellen en Ruby en ook bij de collega's van de slaappolikliniek: Ans, Astrid en Marion en onze PA'er Inge.

Naast hen, bedank ik ook al die collega's op afdelingen, die mij regelmatig vroegen hoe het ging en hoe ver ik nu was. Vrijwel altijd gevolgd door: 'Knap hoor dat je dat nu 
nog doet'. Dit soort complimenten is als de smeerolie in een roestige fiets, het maakte dat ik door kon blijven trappen.

Leonie en Marianne, jullie noemden jezelf al mijn paranimfen toen ik nog maar nauwelijks gestart was. Dat hield in dat we met enige (on)regelmaat een lunch of kopje koffie deelden. Het werd wat serieuzer toen ik als cadeau kledingadvies kreeg, maar jullie waren vooral mijn uitlaatklep. Niet alleen als ik moeite had om mijn evenwicht te bewaren, maar ook bij de succesjes. Het was voor mij dan ook niet meer dan logisch dat jullie ook mijn daadwerkelijke paranimfen zijn.

Lieve Linda, je stond en staat altijd klaar om te helpen. Tijdens het onderzoek was je een zeer secuur bewaker van het randomisatieproces. Je hielp bij de planning, maar vooral had je altijd een luisterend oor waardoor je een enorme steun was in het behouden van mijn balans. Ook alle collega's van het secretariaat wil ik bedanken. Regelmatig werd gevraagd hoe het ging en wat ik nu eigenlijk precies aan het doen was. Het is fijn om te weten dat er meegeleefd wordt.

Alle neurologen van Kempenhaeghe en MUMC+, bedankt voor jullie bijdragen aan mijn onderzoeksproject.

Je evenwicht proberen te bewaren door in beweging te blijven gebeurt niet alleen op het werk. Ook het, steeds groter wordende, thuisfront speelt daarin een belangrijke rol. Het thuisfront is belangrijk voor zij die op missie zijn, nietwaar Bas? Jullie waren mijn thuisfront op mijn missie: het promotietraject. Gijs, Hanneke, Janne, Tim, Bas en Kris, jullie waren er altijd als ik jullie nodig had. Niels, Lars en Marit, als jullie komen word ik vrolijk en ik denk dat oma Loes nu wat meer tijd heeft om met jullie te knutselen, 'slijm' te maken of vliegtuigen te bouwen.

In de eerste jaren van de promotie waren daar Noppes, Slipper en Wobbel. Zij zorgden voor mijn evenwicht en rust. Het is fantastisch om, als je thuis komt, begroet te worden door altijd enthousiaste honden. Aan de andere kant zijn honden ook een verantwoordelijkheid en ik merkte dat pas goed toen jij, Rein, opnieuw in mijn leven kwam en een deel van die verantwoordelijkheden op je nam. Je stimuleerde me om door te zetten als ik er even doorheen zat en zorgde dat ik ook de ruimte kreeg om door te gaan.

Ik denk dat nu de tijd is gekomen om de plannen, die we nog moeten maken voor de komende twintig jaar, ook daadwerkelijk te gaan maken. 


\section{ABOUT THE AUTHOR}

Loes Leenen werd op 24 december 1955 geboren in Amsterdam. Na het behalen van haar VWO-diploma in 1975 aan het Carthesius Lyceum in Amsterdam, verhuisde zij naar Eindhoven waar zij de in-service opleiding tot psychiatrisch verpleegkundige volgden binnen de Rijks Psychiatrische Inrichting. Na deze opleiding, volgde er een carrière als moeder van drie kinderen, zoals gebruikelijk in die tijd. Wel haalde zij haar propedeuse Sociale Wetenschappen aan de Universiteit van Tilburg in 1984.

In 1988 begon zij als verpleegkundige te werken bij het Epilepsiecentrum Kempenhaeghe. Vanaf 1999 werkte zij op de polikliniek en ernaast als researchverpleegkundige.

In 2000 voltooide zij de post-HBO-opleiding Verpleegkundig Specialist (oude stijl) en in 2002 de post-HBO-opleiding Zorginnovatie, beide aan de Hoge School Utrecht. In 2009 behaalde zij haar masterdiploma verpleegkundig specialist. Haar masterthesis bestond uit een praktijkgericht onderzoek. Dit onderzoek betrof een digitale aanvalskalender voor patiënten met epilepsie, wat aan het elektronisch patiëntendossier gekoppeld werd.

Zij heeft deelgenomen aan diverse projecten, waaronder de ontwikkeling van een digitale aanvalskalender en een app voor mensen met epilepsie. Binnen de Nervus vagus stimulatie (NVS) werkgroep heeft zij meegewerkt aan de ontwikkeling van het NVS-handboek.

Zij is betrokken geweest bij de ontwikkeling van diverse scholingsmodules, zo ook in het onderwijsprogramma voor verpleegkundigen waar ze de module risicomanagement verzorgt. Sinds 2000 is zij een van de leden van lokale medisch ethische commissie. Loes is tevens actief in de 'landelijke nascholingsdag voor epilepsiezorgverleners', waar zij verantwoordelijk is voor het onderdeel zelfmanagement.

In 2013 startte zij naast werken op de polikliniek haar promotieonderzoek. Dit onderzoek werd uitgevoerd binnen het departement of Health Services Research van de Universiteit van Maastricht en de afdeling Onderzoek \& Ontwikkeling van het Academisch Centrum Kempenhaeghe. De resultaten van dit onderzoek zijn beschreven in dit proefschrift

In haar huidige werkveld richt zij zich binnen het aandachtsgebied volwassen met epilepsie op 'epilepsie bij ouderen'.

Loes woont in Sterksel met Rein, waar ze graag gaan wandelen met de honden, maar ook knutselen en lezen met de kleinkinderen zijn favoriete bezigheden voor haar. 


\section{English}

Loes Leenen was born on December 24th, 1955 in Amsterdam. After obtaining her VWO diploma in 1975 at the Carthesius Lyceum in Amsterdam, she moved to Eindhoven where she studied to become a psychiatric nurse at the Rijks Psychiatrische Inrichting. After completing her training, she spent the next few years in a new career as mother of three children, during which time she gained a diploma (propaedeutic) in Social Sciences at the University of Tilburg in 1984.

In 1988, she started work as a nurse at the Epilepsy Centre Kempenhaeghe, and since 1999 she has worked at the outpatient clinic and as a research nurse.

In 2000, she completed a post-graduate course on nursing and in 2002 a post-graduate course in health care innovation, both at the University of Applied Sciences. In 2009, she obtained her master's degree as a nurse specialist. Her master's thesis consisted of a practice-oriented study, the focus being the digital seizure calendar for patients with epilepsy, which was linked to the electronic patient record. She also participated in a project to develop a smartphone application for people with epilepsy and within the Nervus vagus stimulation (NVS) working group, she contributed to the development of the NVS manual.

She was involved in the development of various training modules, as well as in the education programme for nurses, where she delivers the risk management module. Since 2000 she has been a member of the local medical ethics committee. Loes is also active in the 'national training day for epilepsy care providers', where she is responsible for the self-management component.

In 2013, she embarked on her PhD while working at the outpatient clinic. This research was conducted within the Department of Health Services Research of the University of Maastricht and the Research \& Development Department of the Academic Centre Kempenhaeghe. The results of the study are described in this thesis

Within her current field of work, epilepsy in adult patients, her particular focus is epilepsy in the elderly.

Loes lives with Rein in Sterksel, where she likes to walk the dogs, but playing with and reading to her grandchildren are her favourite activities. 
